\title{
The Potential for Neutrino Physics at Muon Colliders and Dedicated High Current Muon Storage Rings
}

\author{
I. Bigi, ${ }^{\text {a }}$ T. Bolton, ${ }^{\mathrm{b}}$ J. Formaggio, ${ }^{\mathrm{c}}$ D. A. Harris, ${ }^{\mathrm{d} B}$ B. Kayser, ${ }^{\mathrm{e}}$ \\ B. J. King* ${ }^{f}$ K. S. McFarland, ${ }^{g}$ J. Morfin, ${ }^{\mathrm{d}}$ A. A. Petrov, ${ }^{\mathrm{h}}$ \\ H. Schellman, ${ }^{\text {R R. Shrock, }},{ }^{j}$ P. G. Spentzouris, ${ }^{\mathrm{d}}$ M. Velasco, ${ }^{\mathrm{i}}$ \\ J. $\mathrm{Yu}^{\mathrm{d}}$ \\ ${ }^{a}$ Notre Dame University, South Bend, IN, USA \\ b Kansas State University, Manhattan, KS, USA \\ ${ }^{\mathrm{c}}$ Columbia University, New York, NY, USA \\ ${ }^{\mathrm{d}}$ Fermilab, Batavia, IL, USA \\ e National Science Foundation, Washington, DC, USA \\ ${ }^{\mathrm{f}}$ Brookhaven National Laboratory, Upton, NY, USA \\ ${ }^{\mathrm{g}}$ University of Rochester, Rochester, NY, USA \\ ${ }^{\mathrm{h}}$ LNS, Cornell University, Ithaca, NY, USA \\ ${ }^{\mathrm{i}}$ Northwestern University, Evanston, IL, USA \\ ${ }^{\mathrm{j} S t a t e}$ University of New York, Stonybrook, NY, USA \\ *Contact author; email: bking@bnl.gov
}

\begin{abstract}
Conceptual design studies are underway for muon colliders and other high-current muon storage rings that have the potential to become the first true "neutrino factories". Muon decays in long straight sections of the storage rings would produce precisely characterized beams of electron and muon type neutrinos of unprecedented intensity. This article reviews prospects the for these facilities to greatly extend our capabilities for neutrino experiments, largely emphasizing the physics of neutrino interactions.
\end{abstract}

Key words:

muon colliders, muon storage rings,neutrino factories; PACS13.15.+g

Preprint submitted to Elsevier Preprint 27 October 2018 


\section{Contents}

\begin{tabular}{lll}
\hline 1 & Overview & 6
\end{tabular}

\begin{tabular}{lll}
\hline 1.1 & Introduction & 7
\end{tabular}

\begin{tabular}{lll}
\hline 1.2 & Experimental Overview & 7
\end{tabular}

$\begin{array}{lll}1.3 & \text { Physics Overview } & 16\end{array}$

$2 \quad$ Deep Inelastic Scattering and QCD Studies 22

$2.1 \quad$ Background on Measuring Parton Distribution Functions and QCD $\begin{array}{ll}\text { with Non-Polarized Targets } & 24\end{array}$

$2.2 \quad$ Measurement of Quark Parton Distribution Functions 26

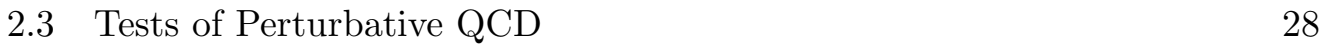

\begin{tabular}{|lll}
2.4 & Heavy Quark Production & 30
\end{tabular}

\begin{tabular}{|lll}
\hline 2.5 & Parton Distribution Functions at Large Bjorken $x$ & 32
\end{tabular}

\begin{tabular}{|lll}
\hline 2.6 & Examining the Spin Structure of the Nucleon & 33
\end{tabular}

$\begin{array}{lll}2.7 & \text { Experimental Setup and Measurement Technique } & 35\end{array}$

$2.8 \quad$ Applications of Polarized Parton Distribution Data from $\nu \mathrm{MCS} \quad 36$

\begin{tabular}{|lll}
\hline 2.9 & Studying Nuclear Effects with Neutrinos & 37
\end{tabular}

$3 \quad$ Studies of the CKM Quark Mixing Matrix 40

\begin{tabular}{lll}
\hline 3.1 & Introduction & 41
\end{tabular}

3.2 Analyses Involving Charm Production: the Extraction of $V_{c d}$ and $V_{c s}$

3.3 Analyses Involving Bottom Production: the Extraction of $V_{u b}$ and $\begin{array}{lll}V_{c b} & 46\end{array}$

$3.4 \quad V_{c d} / V_{c s}$ via Diffractive Charmed Vector Meson Production 48

$3.5 \quad$ Improved Knowledge of the CKM Matrix from $\nu \mathrm{MCs} \quad 48$

\begin{tabular}{|lll}
\hline 4 & Precision Electroweak Studies & 49
\end{tabular}

\begin{tabular}{lll}
\hline 4.1 & Introduction & 49
\end{tabular}

$4.2 \quad$ Elastic and Quasi-Elastic Neutrino-Electron scattering 51 
$\begin{array}{lll}4.3 & \sin ^{2} \theta_{W} \text { from Deep Inelastic Scattering } & 60\end{array}$

\begin{tabular}{|lll}
\hline 4.4 & Summary on $\sin ^{2} \theta_{W}$ Measurements at $\nu \mathrm{MCs}$ & 68
\end{tabular}

$\begin{array}{lll}5 \quad \text { Rare and Exotic Processes } & 69\end{array}$

$\begin{array}{lll}5.1 & \text { New Physics Sensitivity } & 69\end{array}$

\begin{tabular}{|ll}
5.2 & Studies of Low Energy QCD \\
\hline
\end{tabular}

\begin{tabular}{|lll}
5.3 & Conclusions on Rare and Exotic Processes at $\nu \mathrm{MCS}$ & 78
\end{tabular}

\begin{tabular}{lll}
\hline 6 Charm Decay Physics & 78
\end{tabular}

\begin{tabular}{lll}
\hline 6.1 & Introduction & 78
\end{tabular}

$\begin{array}{lll}6.2 & \text { Theoretical Motivation for Charm Physics } & 79\end{array}$

6.3 Probing Strong Interactions through Charm Decays 80

6.4 Searches for New Physics in Charm Decays 84

6.5 Summary on Charm Decay Physics at $\nu \mathrm{MCs}$

\begin{tabular}{|ll|}
\hline 7 Neutrino Oscillation Experiments with a Muon Storage \\
Ring/Neutrino Factory
\end{tabular}

$7.1 \quad$ Status of Neutrino Oscillations at the Time of $\nu \mathrm{MCs}$

\begin{tabular}{lll}
\hline 7.2 & Oscillation Experiments at $\nu \mathrm{MCs}$ & 91
\end{tabular}

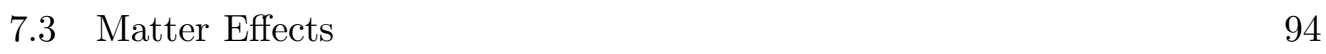

$\begin{array}{lll}7.4 \quad \text { Detector Considerations } & 95\end{array}$

7.5 Conclusions on Neutrino Oscillation Studies at $\nu \mathrm{MCs} \quad 98$

\begin{tabular}{lll}
\hline Summary & 99
\end{tabular}

$\begin{array}{lll}9 & \text { Acknowledgments } & 99\end{array}$

\begin{tabular}{lr}
\hline References & 100
\end{tabular} 


\section{List of Figures}

$1 \quad$ Example neutrino event spectra for $\nu_{\mu}$ and $\bar{\nu}_{e}$ from a $50 \mathrm{GeV}$ negative muon beam from a neutrino factory $10 \mid$. Solid curves indicate the spectra for decays at zero degrees in the center of mass system. This is the spectrum expected for a detector located very far from the muon decay region. The dashed curves indicate the spectra for decays within the forward hemisphere in the center of mass frame. This is what would be expected for a detector close enough to the muon decay region to subtend an angle of $1 / \gamma$.

\begin{tabular}{|ll|l|l|l|}
\hline 2 & Example of a general purpose neutrino detector & 6 & . Its scale \\
\hline
\end{tabular} is illustrated by a human figure in the lower left corner. The neutrino target is the small horizontal cylinder at mid-height on the right hand side of the detector. Its radial extent corresponds roughly to the radial spread of the neutring pencil beam, which is incident from the right hand side. The illustration is partially schematic in that the geometries of the calorimeters and dipole magnet have been simplified for illustrative purposes.

3 Conceptual illustration of the vertex tagging superiority expected at $\nu$ MCs over that with collider experiment geometries. (The figure is reproduced from 14 , which used the terminology "MURINE", for MUon RIng Neutring Experiment, instead of $\nu$ MC.) Neutrino targets could have a vertex plane of CCD pixel detectors spaced at intervals of approximately one millimeter. For comparison, the VXD3 vertexing detector at the SLD experiment at SLAC, generally regarded as the best existing vertex detector in a collider experiment, has its two innermost CCD tracking planes at 2.8 $\mathrm{cm}$ and $3.8 \mathrm{~cm}$ from the interaction point (IP). A schematid of a one-prong $D^{+}$decay has been drawn to illustrate the advantages of closely spaced vertex detectors. For clarity of illustration, the kink deflection angle has been drawn much larger than would be typical. The $2 \mathrm{~cm}$ distance to decay for the $D^{+}$charmed meson corresponds to the average boosted lifetime for a $120 \mathrm{GeV} D^{+}$. 
$4 \quad$ Fractions of the total neutrino-nucleon cross section involving production of heavy flavors in the final state, for an isoscalar target and as a function of neutrino energy 8 . The plotted production fractions are for charged current charm production $\left(R_{c E}\right.$, and $R_{c l o E}$ is the leading order approximation), neutral current production of a charm-anticharm pair $\left(R_{c c E}\right)$, charged current $B$ production from a $u$ quark $\left(R_{b u E}\right.$, again with $R_{\text {buloE }}$ as the leading order approximation), charged current $B$ production from a $c$ quark $\left(R_{b c E}\right)$ and neutral current production of a $b b$ pair $\left(R_{b b E}\right)$.

$5 \quad$ Heavy quark fractional rates vs neutrino energies corresponding to Fig. 4, but for antineutrinos rather than neutrinos $8 \mid$.

$6 \quad$ Model-dependent decomposition of nucleon spin into contributions from quarks and gluons. The two vertical dotted lines show the naive QPM expectation and the results from a NLO fit to most of the available data on $g_{1}^{l}$.

7 Simulated predictions for the ratios of both the $F_{2}$ and $x F_{3}$ structure functions between calcium and deuterium, plotted as a function of Bjorken $x$ and assuming the theoretical model of \begin{tabular}{|l|l|l|l|l}
\hline Kulagin & 45 & and data samples from a $\nu \mathrm{MC}$. The sizes of the \\
\hline
\end{tabular} error bars correspond approximately to 1-year exposures for both targets at the $50 \mathrm{GeV} \nu \mathrm{MC}$ of Table 1.

$8 \quad$ Feynman diagrams contributing to the $\nu-e$ scattering processes of Eqs. 62 to 66: (a) NC $\nu$-e elastic scattering (for Eqs. 62, 64, 65 and 66) (b) CC $\nu_{e} e$ scattering (for Eq. 65), (c) $\mathrm{CC} \bar{\nu}_{e} e$ annihilation (for equation 66 ), and (d) inverse muon decay (for Eq. 64).

$9 \quad$ Integral cross section for neutrino-electron scattering processes above any chosen cut on the inelasticity variable, $y>y_{\text {cut }}$, and assuming $E_{\nu}=30 \mathrm{GeV}$.

10 Change in the integral cross section with respect to $\sin ^{2} \theta_{W}$ for neutrino-electron scattering processes above $y>y_{\text {cut }}$, assuming $E_{\nu}=30 \mathrm{GeV}$. 
11 Statistical uncertainty in $\sin ^{2} \theta_{W}$ for any chosen value of $y_{\text {cut }}$, from neutrino-electron scattering in beams of either $\nu_{\mu}, \nu_{e}, \bar{\nu}_{\mu}$ or $\bar{\nu}_{e}$. An integrated luminosity of $10^{40} \mathrm{~cm}^{-2}$ at a beam energy of $E_{\nu}=30 \mathrm{GeV}$ has been assumed. The values can be scaled to other neutrino energies by noting that the measurement's statistical uncertainty, for a given integrated neutrino flux through a specified detector, is proportional to the inverse square root of the average energy.

12 Same as Fig. 11 except the event statistics has been summed over the experimentally indistinguishable contributions from the two neutrino components in the $\mu^{+}$-induced $\bar{\nu}_{\mu} \nu_{e}$ beams and the $\mu^{-}$-induced $\nu_{\mu} \bar{\nu}_{e}$ beams produced from muon storage rings.

13 Monte-carlo generated simulation of a high energy neutrinoelectron scattering interaction in liquid helium. The distorted scale is indicated by the 10:1 ratio in the relative lengths of the $\mathrm{x}$ and $\mathrm{z}$ axes. The solid lines are electrons and positrons and the dashed lines are photons that would not be seen in the detector. The view is perpendicular to both the beam direction and to a 0.1 Tesla magnetic field. See text for further details.

14 Expected future restrictions in the size of the allowed regions in a plot of $W$ mass vs. top quark mass, following experiments at TeV 33 and at a $\nu M C$, as illustrated by the thicknesses of the bands and ellipse. The exact positions of the shaded regions are for illustrative purposes only.

15 Plots for limits on the (from top to bottom) coupling values

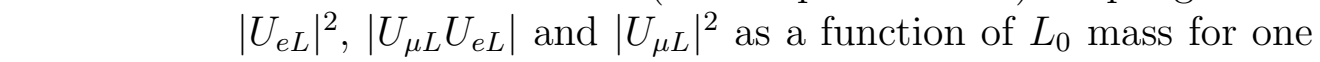
year of running under the assumptions given in the text. The solid, dashed, and dotted lines represent $\nu \mathrm{MC}$ energies of 10 , 20 , and $250 \mathrm{GeV}$ respectively. The plots on the left show limits for $L=12 \mathrm{~m}$, and the plots on the right show limits for $L=1$ $\mathrm{km}$.

16 The upper plot shows $\nu_{e}$ and $\bar{\nu}_{\mu}$ event rates per GeV as a function of $\mathrm{E} / \mathrm{L}$ for two different experiments. The lower plot shows the L/E dependence of the oscillation probability, assuming the largest $\delta m^{2}$ is $2,3.5$,or $5 \times 10^{-3} \mathrm{eV}^{2} / c^{4}$.

\section{Overview}




\subsection{Introduction}

Muon colliders have been proposed to provide lepton-lepton collisions while circumventing the energy limitations on electron-positron storage rings caused by synchrotron radiation. The larger muon mass suppresses synchrotron radiation energy losses by a factor $m_{e}^{4} / m_{\mu}^{4} \simeq 5 \times 10^{-10}$ relative to those of a circulating electron beam of the same energy and, incidentally, also opens up promising possibilities for $s$-channel Higgs boson production[1].

Recent feasibility and design studies for future muon colliders [2,1] have begun to focus attention on the exciting physics possibilities for experiments using neutrino beams from the decays of the circulating high energy muons. This report explores the potential for a "neutrino experiment at a muon collider", or $\nu \mathrm{MC}$ for short. A $\nu \mathrm{MC}$ program could operate either parasitically during a colliding beam experiment; or it could be installed as part of a program in neutrino physics at a dedicated muon storage ring.

Amongst the potential physics topics for $\nu \mathrm{MCs}$, neutrino oscillations have garnered the most intense experimental and theoretical activity, and particular possibilities for long baseline oscillation experiments exploiting a muon storage ring are covered elsewhere[3,4]. In this report, we wish to also highlight the superb capabilities of neutrinos as probes of the strong and weak interaction dynamics of quarks and the parton structure of nucleons, as well as the power of a $\nu \mathrm{MC}$ in searches for evidence of new types of weak interactions.

The remainder of this section lays out the expected experimental parameters and capabilities of a $\nu \mathrm{MC}$ and provides concise overviews for the more detailed physics discussions that follow.

\subsection{Experimental Overview}

\subsubsection{High Current Muon Storage Rings}

Recent ideas for neutrino experiments at either muon colliders $[5,6]$ or dedicated neutrino factories[7] represent reincarnations of earlier proposals for neutrino experiments at muon storage rings that date back at least to the 1960's. The essential advantages of modern $\nu \mathrm{MCs}$ derive from the very large muon currents that might be produced and stored using the technologies developed for muon colliders. Current design scenarios for muon colliders[2,1] and

neutrino factories $[10,4]$ envision of order $10^{21}$ positive and negative muons per year circulating and decaying in the storage ring.

Neutrinos from decays in the longest straight sections of the storage ring will 


\begin{tabular}{|c|c|c|c|c|c|}
\hline description & \multicolumn{2}{|c|}{$\nu$-factory } & Higgs-factory & \multicolumn{2}{|c|}{ top-factory } \\
\hline muon energy, $E_{\mu}$ & \multicolumn{2}{|c|}{$20 \mathrm{GeV}$} & $50 \mathrm{GeV}$ & \multicolumn{2}{|c|}{$175 \mathrm{GeV}$} \\
\hline$\mu^{ \pm} /$year $\left[N_{\mu} / 10^{20}\right]$ & \multicolumn{2}{|c|}{3.0} & 6.0 & \multicolumn{2}{|c|}{6.0} \\
\hline flight time to beam dump $\left|t_{D} / \gamma \tau_{\mu}\right|$ & \multicolumn{2}{|c|}{ no dump } & no dump & \multicolumn{2}{|c|}{ no dump } \\
\hline ring circumference, $C[\mathrm{~m}]$ & \multicolumn{2}{|c|}{300} & 345 & \multicolumn{2}{|c|}{900} \\
\hline straight section $(\mathrm{SS})$ length $l_{s s}[\mathrm{~m}]$ & \multicolumn{2}{|c|}{90} & 40 & \multicolumn{2}{|c|}{110} \\
\hline fractional SS length, $\left[f_{s s} \equiv l_{s s} / C\right]$ & \multicolumn{2}{|c|}{0.30} & 0.12 & \multicolumn{2}{|c|}{0.12} \\
\hline$\mu^{+} /$year in SS, $\left[N_{\mu}^{s s} \equiv f_{s s} N_{\mu} / 10^{20}\right]$ & \multicolumn{2}{|c|}{0.90} & 0.72 & \multicolumn{2}{|c|}{0.72} \\
\hline$\nu$ from SS/year $\left[/ 10^{20}\right]$ & \multicolumn{2}{|c|}{1.8} & 1.4 & \multicolumn{2}{|c|}{1.4} \\
\hline$\nu$ angular divergence $\left[/ \gamma \cdot \delta \theta_{\nu}\right]$ & \multicolumn{2}{|c|}{1} & 1 & \multicolumn{2}{|c|}{1} \\
\hline$\nu$ angular divergence $[\mathrm{mrad}]$ & \multicolumn{2}{|c|}{5.3} & 2.1 & & 60 \\
\hline$N^{s b}\left[\right.$ events $\left./ \mathrm{yr} / \mathrm{g} / \mathrm{cm}^{2}\right]$ & $3.8>$ & $10^{6}$ & $6.5 \times 10^{6}$ & $2.7>$ & $\times 10^{7}$ \\
\hline target thickness $\left[\mathrm{g} \mathrm{cm}^{-2}\right]$ for $10^{10}$ events & & & 1500 & & 70 \\
\hline$N^{l b}\left[\right.$ events $\left./ \mathrm{yr} / \mathrm{kT} /\left(10^{3} \mathrm{~km}\right)^{2}\right]$ & 1.2 & $10^{4}$ & $1.4 \times 10^{5}$ & $6.2>$ & $\times 10^{6}$ \\
\hline description & & frontier & 2nd gene & ation & \\
\hline muon energy, $E_{\mu}$ & & $500 \mathrm{GeV}$ & $5 \mathrm{Te}$ & & \\
\hline$\mu^{ \pm} /$year $\left[N_{\mu} /\right.$ & & 3.2 & 3.6 & & \\
\hline flight time to beam dump $\left[t_{D} /\right.$ & & 0.5 & no du & & \\
\hline ring circumference, & $C[\mathrm{~m}]$ & 2000 & 150 & & \\
\hline straight section (SS) length & $s[\mathrm{~m}]$ & 150 & 450 & & \\
\hline fractional SS length $\left[f_{s s} \equiv l\right.$ & $s / C]$ & 0.12 & 0.0 & & \\
\hline$\mu^{+} /$year in SS $\left[N_{\mu}^{s s} \equiv f_{s s} N_{\mu} /\right.$ & $0^{20}$ & 0.38 & 0.1 & & \\
\hline$\nu$ from $\mathrm{SS} /$ year $[/$ & $\left.0^{20}\right]$ & 0.30 & 0.2 & & \\
\hline$\nu$ angular divergence $[/ \gamma$ & $\left.\delta \theta_{\nu}\right]$ & 10 & 1 & & \\
\hline$\nu$ angular divergence $[\mathrm{r}$ & $\mathrm{rad}]$ & 2.1 & 0.02 & & \\
\hline$N^{s b}[$ events $/ \mathrm{yr} / \mathrm{g}$ & $\left.\mathrm{cm}^{2}\right]$ & $2.3 \times 10^{7}$ & $1.0 \times$ & $10^{8}$ & \\
\hline target thickness $\left[\mathrm{g} \mathrm{cm}^{-2}\right]$ for $10^{10} \mathrm{e}$ & rents & 430 & 10 & & \\
\hline$N^{l b}\left[\right.$ events $/ \mathrm{yr} / \mathrm{kT} /\left(10^{3} \mathrm{l}\right.$ & $\left.m)^{2}\right]$ & $5.0 \times 10^{5}$ & $2.2 \times$ & $0^{10}$ & \\
\hline
\end{tabular}

Table 1

Neutrino fluxes and event rates for representative example parameter sets for dedicated neutrino factories or muon colliders[9] spanning the energy range $E_{\mu}=20$ $\mathrm{GeV}$ to $5 \mathrm{TeV}$. The angular divergence scaling factor, $\gamma \cdot \delta \theta$, is the factor by which the divergence of the parent muon beam increases the neutrino beam's angular divergence beyond the characteristic size, $\delta \theta=1 / \gamma$, expected for a divergenceless muon beam. 


\section{Neutrino Fluxes from a $50 \mathrm{GeV}$ Muon Beam}

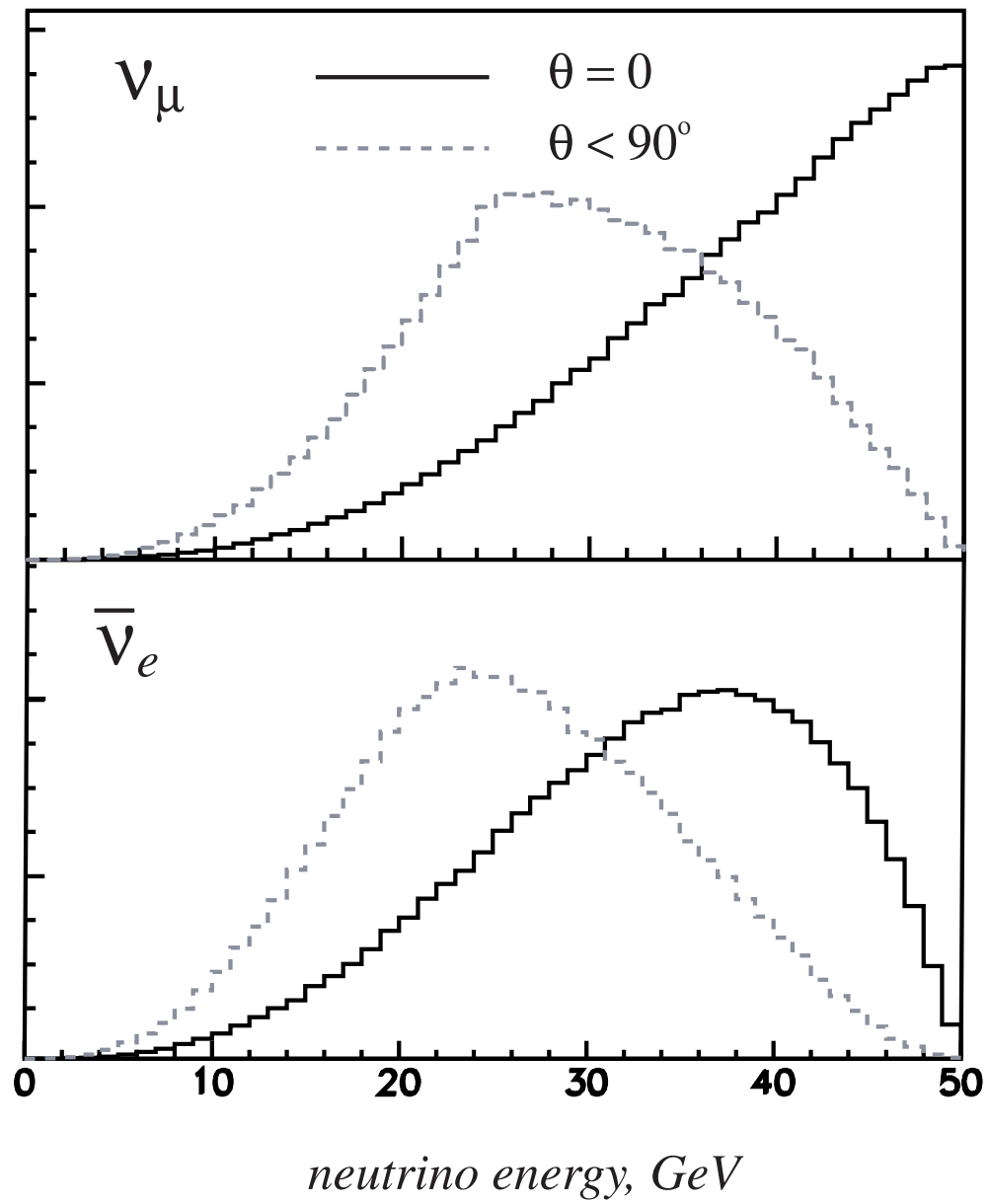

Fig. 1. Example neutrino event spectra for $\nu_{\mu}$ and $\bar{\nu}_{e}$ from a $50 \mathrm{GeV}$ negative muon beam from a neutrino factory[10]. Solid curves indicate the spectra for decays at zero degrees in the center of mass system. This is the spectrum expected for a detector located very far from the muon decay region. The dashed curves indicate the spectra for decays within the forward hemisphere in the center of mass frame. This is what would be expected for a detector close enough to the muon decay region to subtend an angle of $1 / \gamma$.

emerge in intense collinear beams that are highly suitable for experiments. Beams from such production straight sections should provide many orders of magnitude higher event rates than considered in the early versions of muon storage rings and, indeed, should be considerably more intense than today's "conventional" neutrino beams produced from $\pi / K$ decays. No need exists for a conventional beam's muon shielding berm, and detectors can be placed relatively close to the end of the production straight section. Coupled with the relativistic kinematics of muon decay, this permits the possibility of detectors only tens of centimeters across and allows for the use of high precision vertex detectors as active neutrino targets. 


\begin{tabular}{|r|ccc|}
\hline target purpose & general & polarized & $\nu-e$ \\
\hline material & Si CCD & solid $H D$ & liquid $C H_{4}$ \\
mean density $\left[\mathrm{g} / \mathrm{cm}^{3}\right]$ & 0.5 & 0.267 & 0.717 \\
length $[\mathrm{m}]$ & 2 & 0.5 & 20 \\
thickness $\left[\mathrm{g} \mathrm{cm}^{-2}\right]$ & 100 & 13.4 & 1430 \\
radius $[\mathrm{cm}]$ & 20 & 20 & 20 \\
mass $[\mathrm{kg}]$ & 126 & 16.8 & 1800 \\
integrated luminosity $[\mathrm{fb}-1]$ & $6.0 \times 10^{6}$ & $8.1 \times 10^{5}$ & $8.6 \times 10^{7}$ \\
DIS events/year at $50 \mathrm{GeV}$ & $7.7 \times 10^{8}$ & $1.0 \times 10^{8}$ & $1.1 \times 10^{10}$ \\
DIS events/year at $175 \mathrm{GeV}$ & $2.7 \times 10^{9}$ & $3.6 \times 10^{8}$ & $3.8 \times 10^{10}$ \\
$\nu_{e}$ events/year at $50 \mathrm{GeV}$ & $2 \times 10^{5}$ & $\mathrm{NA}$ & $3 \times 10^{6}$ \\
$\nu_{e}$ events/year at $175 \mathrm{GeV}$ & $7 \times 10^{6}$ & $\mathrm{NA}$ & $1 \times 10^{7}$ \\
\hline
\end{tabular}

Table 2

Specifications, integrated luminosities and event rates for the high rate neutrino targets discussed in this report, assuming the $50 \mathrm{GeV}$ and $175 \mathrm{GeV}$ muon storage ring parameters of Table 1 . The target is assumed to be situated $100 \mathrm{~m}(350 \mathrm{~m})$ downstream from the center of the $50 \mathrm{GeV}(175 \mathrm{GeV})$ production straight section.

Additional physics advantages over $\pi / K$ decay neutrino beams will result from the unique and precisely understood flux composition of the $\nu \mathrm{MC}$ beams. Negative and positive muons decay according to

$$
\begin{aligned}
& \mu^{-} \rightarrow \nu_{\mu}+\bar{\nu}_{e}+e^{-} \\
& \mu^{+} \rightarrow \bar{\nu}_{\mu}+\nu_{e}+e^{+}
\end{aligned}
$$

producing pure 2-component neutrino beams $\square$ via the perhaps best understood of all weak decay processes. These beams will be designated as $\nu_{\mu} \bar{\nu}_{e}$ or $\bar{\nu}_{\mu} \nu_{e}$, respectively, in the rest of this report.

Experimental requirements for the two broad classes of neutrino physics at $\nu \mathrm{MCs}$ differ greatly, chiefly because the experiments would be conducted at very different baseline distances from beam production to the detector. Experiments for neutrino interaction physics will be conducted as close to the muon ring as possible ("short baseline") in order to maximize event rates and to subtend the neutrino beam with a target of small transverse cross section. On the other hand, the choice of baseline for neutrino oscillation studies

$\overline{1}$ We implicitly assume here the absence of a significant lepton family number violating decay of the type $\mu^{-} \rightarrow e^{-} \nu_{e} \bar{\nu}_{\mu}$ but caution that the current experimental limit on the branching fraction for this decay is only $1.5 \%$. This limit will clearly be greatly improved upon from the consistency of the observed $\nu \mathrm{MC}$ spectra with predictions. 
will be dictated by the specific range of possible oscillation parameters under investigation, as discussed further in Chapter 7. Oscillation parameters of current interest motivate the use of very long baselines, even extending to the possibility of transcontinental experiments [7].

As an important caveat on the contents of this report, it should always be borne in mind that the ambitious technologies of these high current muon storage rings still only exist at the feasibility or early design study stage and it is by no means guaranteed that realizable devices will appear anytime soon. Nevertheless, recent progress has been impressive, and the pace of R\&D is accelerating. The reader is referred to the specialist literature for a more thorough overview of the technological challenges in building a muon collider[2,1] or neutrino factory $[10,4]$.

\subsubsection{Event Rates}

Event rates in all $\nu \mathrm{MC}$ experiments will be dominated by the charged current (CC) and neutral current (NC) deep inelastic scattering (DIS) of neutrinos or antineutrinos with nucleons $(N=p$ or $n)$ :

$$
\begin{aligned}
\nu_{\ell}+N & \rightarrow \ell^{-}+X & \left(\nu_{\ell}-\mathrm{CC}\right), \\
\bar{\nu}_{\ell}+N & \rightarrow \ell^{+}+X & \left(\bar{\nu}_{\ell} \mathrm{CC}\right), \\
\nu_{\ell}\left(\bar{\nu}_{\ell}\right)+N & \rightarrow \nu_{\ell}\left(\bar{\nu}_{\ell}\right)+X & \left(\nu_{\ell}\left(\bar{\nu}_{\ell}\right)-\mathrm{NC}\right),
\end{aligned}
$$

where $\ell=e$ or $\mu$ and $X$ represents a typically multi-particle hadronic final state. Neutrino-nucleon DIS cross sections scale with neutrino energy $E_{\nu}$ to a good approximation for neutrino energies above a few $\mathrm{GeV}$, with numerical values of [12]:

$$
\sigma_{\nu N}\left(\begin{array}{c}
\nu-C C \\
\nu-N C \\
\bar{\nu}-C C \\
\bar{\nu}-N C
\end{array}\right) \simeq\left(\begin{array}{c}
6.8 \\
2.1 \\
3.4 \\
1.3
\end{array}\right) \times E_{\nu}[\mathrm{GeV}] \mathrm{fb}
$$

At the many-GeV energies of $\nu \mathrm{MCs}, \nu N$ DIS is well described as the quasielastic scattering of neutrinos off one of the many quarks or antiquarks inside the nucleon through the exchange of a virtual $W$ or $Z$ boson:

$$
\begin{array}{ccc}
\nu_{\ell}\left(\bar{\nu}_{\ell}\right)+q \rightarrow \nu_{\ell}\left(\bar{\nu}_{\ell}\right)+q & (\mathrm{NC}), \\
\nu_{\ell}+q_{d}\left(\bar{q}_{u}\right) \rightarrow \ell^{-}+q_{u}^{\prime}\left(\bar{q}_{d}^{\prime}\right) & (\nu-\mathrm{CC}), \\
\bar{\nu}_{\ell}+q_{u}\left(\bar{q}_{d}\right) \rightarrow \ell^{+}+q_{d}^{\prime}\left(\bar{q}_{u}^{\prime}\right) & (\bar{\nu}-\mathrm{CC}) .
\end{array}
$$


All quarks $q$ participate in the NC process. The CC interactions change quark flavor, with neutrino interactions producing $u$-type and $\bar{d}$-type final state quarks, $q_{u}^{\prime}$ and $\bar{q}_{d}^{\prime}$, from $d$-type and $\bar{u}$-type targets, $q_{d}$ and $\bar{q}_{u}$. Antineutrinos participate in the charge-conjugate processes. Much of the richness of neutrino interaction physics derives from the variety of processes contained in Eqs. 4-6.

\subsubsection{Neutrino Production Spectra and Event Rates in Detectors}

Neutrino flux spectra at $\nu$ MCs will be precisely predictable since the decay of muons is a well-understood purely electroweak process. Characteristics of the parent muon beam in the production straight section can be reliably calculated and modeled through a knowledge of the focusing magnet lattice and through beam monitoring. Calibration of the muon energies in the storage ring might reach the level of a few parts per million fractional uncertainty [11].

Due to the differing angular coverages, the neutrino spectrum seen by an oscillation detector at a long baseline will differ from that seen by detectors placed at short baselines to study interaction physics. Long baseline detectors will sample the very forward-going neutrinos, at angles in the muon rest frame $\left(\theta^{\prime}\right)$ and laboratory frame $(\theta)$ close to $\theta^{\prime}=\theta=0$, while detectors close to the production straight section will instead accept a production solid angle bite that is comparable to the boosted forward hemisphere of the decaying neutrinos,

$$
\theta^{\prime}=\frac{\pi}{2} \Leftrightarrow \theta_{\nu} \simeq \sin \theta_{\nu}=1 / \gamma=\frac{m_{\mu} c^{2}}{E_{\mu}} \simeq \frac{10^{-4}}{E_{\mu}[\mathrm{TeV}]}
$$

Figure 1 gives an illustrative example of the neutrino spectra at $\nu \mathrm{MCs}$ for detectors at both short and long baselines[10], and Table 1 gives beam and event rate parameters for several other $\nu \mathrm{MC}$ scenarios. Further explanation for the choices of storage ring parameters in Table 1 and a derivation for the following simple numerical expressions for event rates used to fill Table 2 are provided elsewhere[9].

For short baseline detectors,

$$
N^{s b}\left[\text { events } / \mathrm{yr} / \mathrm{g} / \mathrm{cm}^{-2}\right]=2.1 \times 10^{-15} \times E_{\mu}[\mathrm{GeV}] \times N_{\mu}^{s s}\left[\mathrm{yr}^{-1}\right]
$$

where $N^{s b}$ is the number of neutrino interactions per year of running per $\mathrm{g} \cdot \mathrm{cm}^{-2}$ of a cylindrically symmetric target centered on the beam, $E_{\mu}$ is the muon beam energy and $N_{\mu}^{s s}$ is the number of forward-going muons (as opposed to muons circulating in the opposite direction in, e.g., a collider ring) 
decaying in the production straight section per year. Equation 8 assumes the parent muon beam to have an angular divergence in the production straight section that is small compared to the $\delta \theta_{\nu}=1 / \gamma$ natural divergence of the neutrino beam. This will normally be the case [13] unless the choice of straight section is in the final focus region of a collider storage ring. Table 2 accounts simplistically for this exception (in this case for the illustrative parameter set at $500 \mathrm{GeV}$ ) by increasing the angular divergence of the neutrino beam by a simple scale factor. In this circumstance, the angular coverage of the target would need to be increased by this same scale factor in order to retain the event rate predicted by the parameter $N^{s b}$.

In contrast to short baseline detectors, the event rate in long baseline detectors is not sensitive to the geometry of the detector since the entire detector will always be bathed uniformly by the forward-going neutrino flux. The number of interactions in the detector will vary in proportion to the target mass $M$ and inversely as the square of the baseline length $L$. This leads to a definition, analogous to Eq. 8, for the event rate benchmark $N^{l b}$ :

$$
N^{l b}\left[\text { events } / \mathrm{yr} / \mathrm{kT} /\left(10^{3} \mathrm{~km}\right)^{-2}\right]=\frac{1.6 \times 10^{-20} \times N_{\mu}^{s s}\left[y r^{-1}\right] \times\left(E_{\mu}[G e V]\right)^{3}}{(\gamma \cdot \delta \theta)^{2}}
$$

where $N^{l b}$ is the number of neutrino interactions per kiloton·year of running with a target centered on the beam at a $1000 \mathrm{~km}$ distance from the production point. The previously discussed angular divergence scaling factor, $\gamma \cdot \delta \theta$, has been explicitly included.

The event rates given in Table 2 are truly impressive. Samples of thousands of events per kiloton might be recorded at oscillation experiments with baselines as long as thousands of kilometers. For neutrino interaction physics, samples as large as 10 billion events can be reasonably contemplated in compact targets close to the production straight section. Equation 7 shows that the radial extent of such targets can be as small 10-20 cm.

\subsubsection{Detector Design Considerations for $\nu M C s$}

Event rates for oscillation experiments will probably be less of an extrapolation from today's experiments than will be the case for interaction experiments due to the compensating rate decrease at the expected longer baselines. Correspondingly, the innovations in neutrino detector design required to upgrade to the neutrino beams at $\nu \mathrm{MCs}$ are likely to be rather less substantial for oscillation experiments at long baselines than for interaction physics experiments.

Two significant changes expected for the design of oscillation detectors for $\nu \mathrm{MCs}$ are that (i) the 2-component beams provide strong motivation for a 


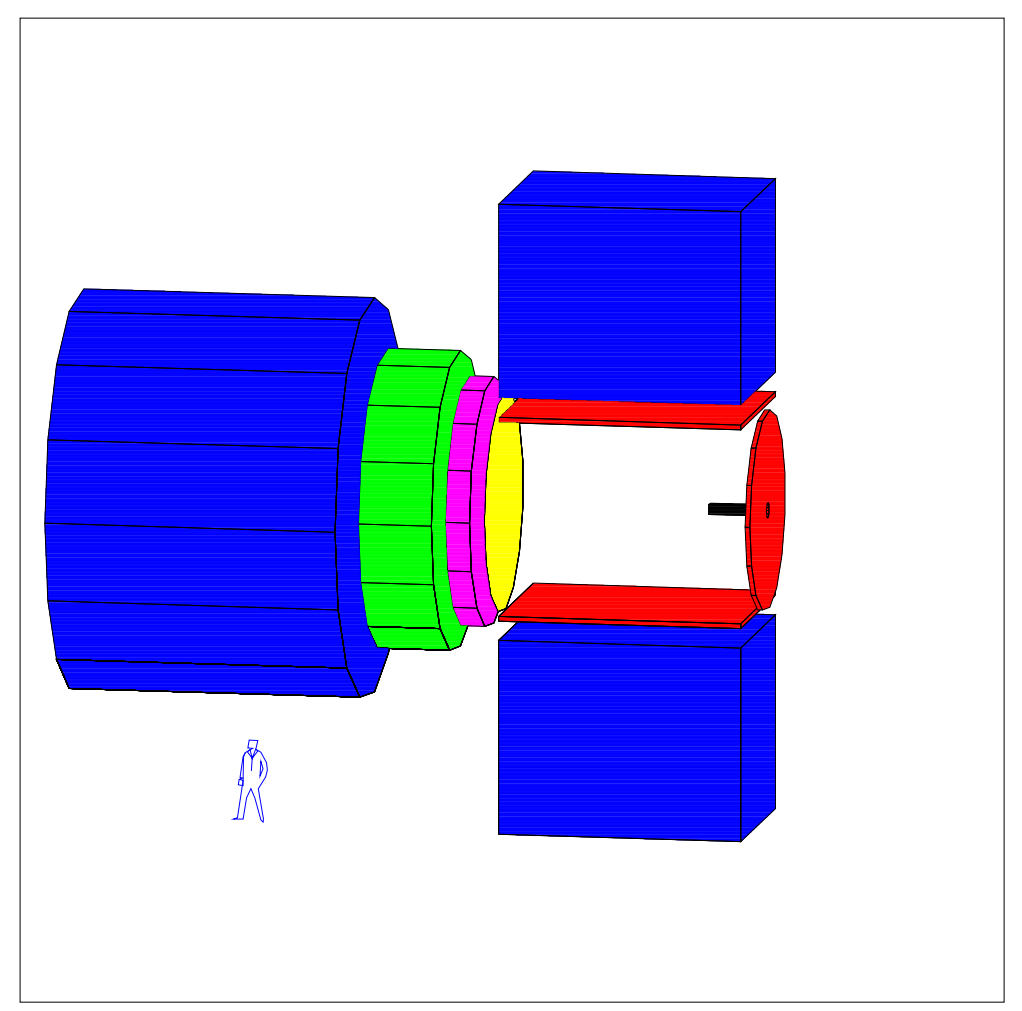

Fig. 2. Example of a general purpose neutrino detector [6]. Its scale is illustrated by a human figure in the lower left corner. The neutrino target is the small horizontal cylinder at mid-height on the right hand side of the detector. Its radial extent corresponds roughly to the radial spread of the neutrino pencil beam, which is incident from the right hand side. The illustration is partially schematic in that the geometries of the calorimeters and dipole magnet have been simplified for illustrative purposes.

magnetic spectrometer to distinguish muon charge signs; and (ii) larger detector masses might be financially justified in order to fully exploit the large financial investment in the muon storage ring. Design considerations for detectors for oscillation $\nu$ MCs are discussed in more detail in Chapter 7.

In contrast to oscillation experiments, the increase in neutrino yield for $\nu \mathrm{MCs}$ relative to beams from pion decays as well as the collimation of the neutrino beams will allow the use of compact, specialized targets surrounded by high performance detectors. These detectors must operate at high rate in order to cope with the data sets implied in Table 1. Considerable thought must be given to triggering, data acquisition, event reconstruction and data handling considerations.

Figure 2 provides an example[6,9] of the sort of high rate general purpose neutrino detector that would be well matched to the intense neutrino beams at $\nu \mathrm{MCs}$. The neutrino target is one meter long stack of CCD tracking planes represented by the small horizontal cylinder at mid-height on the right-hand 


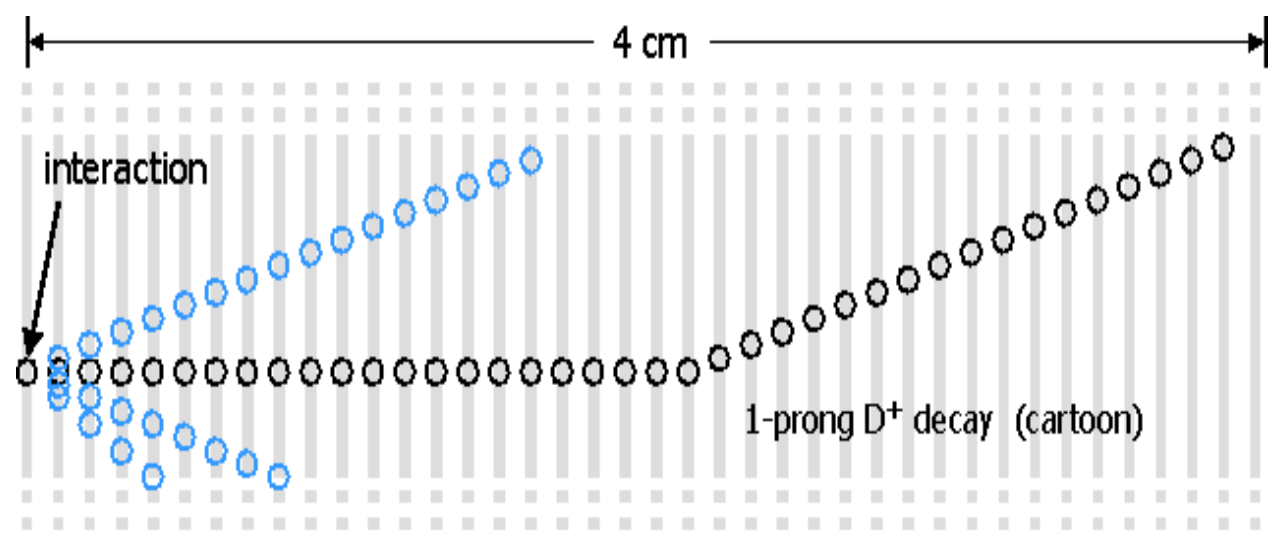

A) vertexing at MURINE

\section{B) best collider vertexing so far : (SD detector, same scale)}

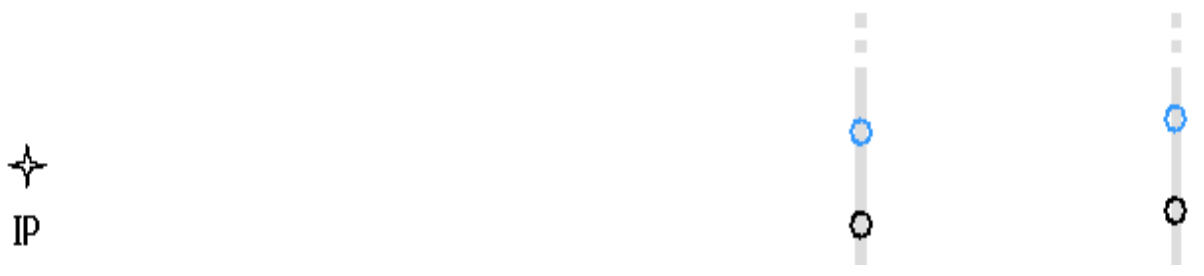

Fig. 3. Conceptual illustration of the vertex tagging superiority expected at $\nu \mathrm{MCs}$ over that with collider experiment geometries. (The figure is reproduced from [14], which used the terminology "MURINE", for MUon RIng Neutrino Experiment, instead of $\nu \mathrm{MC}$.) Neutrino targets could have a vertex plane of CCD pixel detectors spaced at intervals of approximately one millimeter. For comparison, the VXD3 vertexing detector at the SLD experiment at SLAC, generally regarded as the best existing vertex detector in a collider experiment, has its two innermost CCD tracking planes at $2.8 \mathrm{~cm}$ and $3.8 \mathrm{~cm}$ from the interaction point (IP). A schematic of a one-prong $D^{+}$decay has been drawn to illustrate the advantages of closely spaced vertex detectors. For clarity of illustration, the kink deflection angle has been drawn much larger than would be typical. The $2 \mathrm{~cm}$ distance to decay for the $D^{+}$charmed meson corresponds to the average boosted lifetime for a $120 \mathrm{GeV} D^{+}$.

side of the detector in Fig. 2. Its $10 \mathrm{~cm}$ radial extent could correspond to, e.g., the $0.2 \mathrm{mrad}$ divergence of the neutrino beam originating from a $500 \mathrm{GeV}$ muon beam $500 \mathrm{~m}$ upstream of the target. The scale of the entire detector is illustrated by a human figure in the lower left corner, emphasizing the striking contrast in target size with the kiloton-scale coarse-sampling calorimetric targets often used for past and present high rate neutrino experiments.

The CCD target in Fig. 2 contains 750 planes of 300 micron thick silicon CCD's, corresponding to a mass per unit area of approximately $50 \mathrm{~g} \cdot \mathrm{cm}^{-2}$; this translates to 2.5 radiation lengths or 0.5 interaction lengths. Scaling to different target lengths and radii should be straightforward without altering the basic design of the surrounding detector. 
Besides providing the mass for neutrino interactions, the tracking target allows for precise reconstruction of the event topologies from charged tracks, including event-by-event vertex tagging and reconstruction of those interactions containing heavy flavor final states. The fixed target geometry of $\nu \mathrm{MC}$ vertex detectors allows for much more frequent sampling than is possible in collider detectors: Fig. 3 gives a schematic comparison between the charm vertexing capabilities of the CCD detector of Fig. 2 and the current best vertexing detector in a collider experiment[14].

The CCD target is backed by a hermetic detector reminiscent of many collider detector designs. An enveloping time projection chamber (TPC) provides track-following, momentum measurements, and particle identification for essentially all charged tracks emanating from the interactions. Optionally, further particle identification might be available from a mirror that reflects Cherenkov light to an instrumented back-plane directly upstream from the target. The mirror is backed by electromagnetic and hadronic calorimeters and, lastly, by iron-core toroidal magnets for muon identification.

Other possible specialized high rate neutrino target and detector possibilities include polarized solid protium-deuterium targets for spin physics (Sec. 2.6) and nuclear targets (Sec. 2.9) for studies of $A$ dependence. A more massive tracking liquid target (Sec. 4) would be suitable for precision electroweak physics using neutrino-electron scattering. Table 2 provides a summary of some of the characteristics for examples of each of the three high rate target types discussed in this section and also gives plausible but very approximate integrated luminosities and event sample sizes for of the illustrative $50 \mathrm{GeV}$ and $175 \mathrm{GeV}$ beam parameters in Table 1.

\subsection{Physics Overview}

This overview motivates and introduces the more detailed discussions that follow on: deep inelastic scattering and quantum chromodynamics (Sec. 2), quark mixing (Sec. 3), precision electroweak tests (Sec. 4), rare and exotic processes (Sec. 5), charm physics (Sec. 6) and neutrino oscillations (Sec. 7).

Before proceeding, we note that much of the interesting physics involves aspects of $\mathrm{CC}$ and $\mathrm{NC}$ charm and beauty production (and hence the motivation for active vertex detectors as targets).

As well as a CKM physics program that complements those from $B$ and $K$ factories and from precision $W$ boson branching fraction measurements at colliders, $b$ and $c$ production at a $\nu \mathrm{MC}$ allows precisions tests of QCD near heavy flavor thresholds, permits sensitive probes for new physics such as flavorchanging neutral currents, and provides a novel, very high statistics sample of 
charmed hadrons.

Figures 4 and 5 show heavy quark production fractions and indicate that, given the expected multi-billion inclusive event samples, very high statistics can indeed be accumulated for both $c$ and $b$ final states at sufficiently above the relevant energy thresholds.

\subsubsection{QCD and Deep Inelastic Scattering}

Historically, neutrino experiments have made major contributions to our understanding and verification of the both the QCD theory of strong interactions and the constituent components of protons and neutrons. The extrapolation of present experimental statistics consisting of $10^{6-7}$ events to the expected $10^{9-10}$ well reconstructed DIS events at $\nu$ MCs might well provide the best ever experimental laboratory for studying QCD and the structure of the nucleon through a scattering process.

Both traditional and novel areas for potential study will be discussed in Sec. 2 . They include: (1) one of the most precise and theoretically sound measurements of the strong coupling constant, $\alpha_{s}$; (2) stringent consistency checks for the predictions of perturbative QCD; (3) detailed flavor and spin dependence nucleon structure functions using both CC and NC probes; (4) precise tests of QCD near the $c$ and $b$ quark heavy flavor transitions; and (5) the first systematic studies of QCD in nuclear environments probed by neutrino and antineutrino beams.

\subsubsection{The CKM Quark Mixing Matrix}

Some of the most important high-rate measurements will involve the CabibboKobayashi-Maskawa (CKM) mixing matrix that characterizes CC weak interactions of quarks. This topic is discussed in detail in Sec. 3.

Neutrino-nucleon DIS offers unique and systematically independent measurements of CKM matrix elements since it uses a high $Q^{2}$ virtual $W$ probe coupling directly to quarks rather than relying on the complex interplay of weak and strong interactions that is inherent in hadron decay. For sufficiently high energy and event rates, four of the nine CKM matrix element amplitudes $\left|V_{c d}\right|,\left|V_{c s}\right|,\left|V_{u b}\right|$ and $\left|V_{c b}\right|$ - are directly probed through $c$ and $b$ production.

The higher momentum transfers from the external $W$ probe allow for a cleaner theoretical interpretation that requires only relatively small corrections from perturbative QCD. As a further theoretical advantage, the measurements are semi-inclusive - i.e. summing over all final states with single charm or beauty production - and thus do not suffer from uncertainties in hadronic branching 


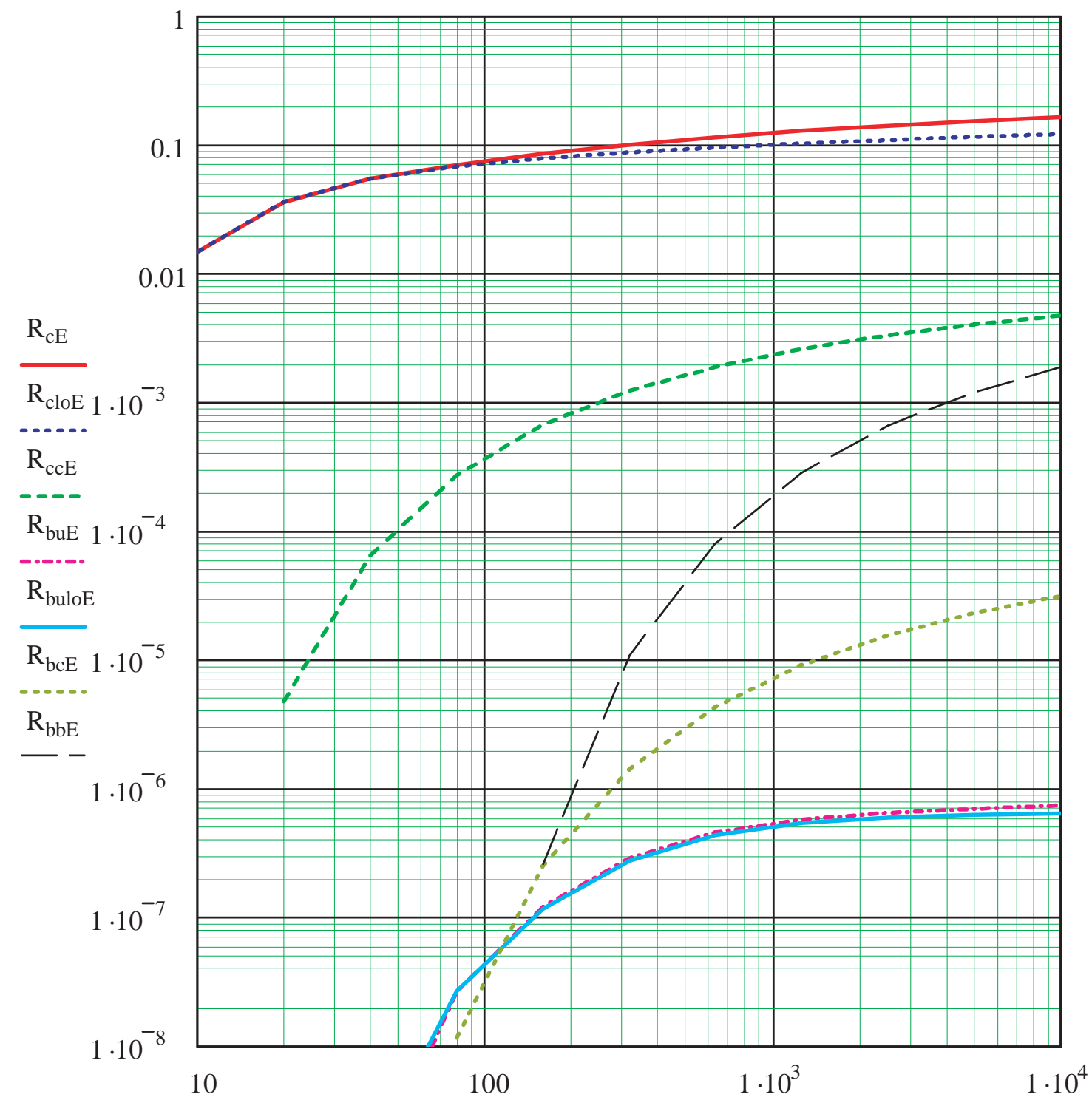

Fig. 4. Fractions of the total neutrino-nucleon cross section involving production of heavy flavors in the final state, for an isoscalar target and as a function of neutrino energy[8]. The plotted production fractions are for charged current charm production $\left(R_{c E}\right.$, and $R_{c l o E}$ is the leading order approximation), neutral current production of a charm-anticharm pair $\left(R_{c c E}\right)$, charged current $B$ production from a $u$ quark ( $R_{b u E}$, again with $R_{b u l o E}$ as the leading order approximation), charged current $B$ production from a $c$ quark $\left(R_{b c E}\right)$ and neutral current production of a $b \bar{b}$ pair $\left(R_{b b E}\right)$.

ratios.

The fractional production rates shown in figures 4 and 5 for $\nu-\mathrm{CC} c$ and $\bar{b}$ production at high energies are of order $\left|V_{c d}\right|^{2}$ and $\left|V_{u b}\right|^{2}$, respectively, where $\left(V_{c d}, V_{u b}\right)$ are the $(d \rightarrow c, u \rightarrow b)$ Cabibbo-Kobayashi-Maskawa (CKM) matrix 


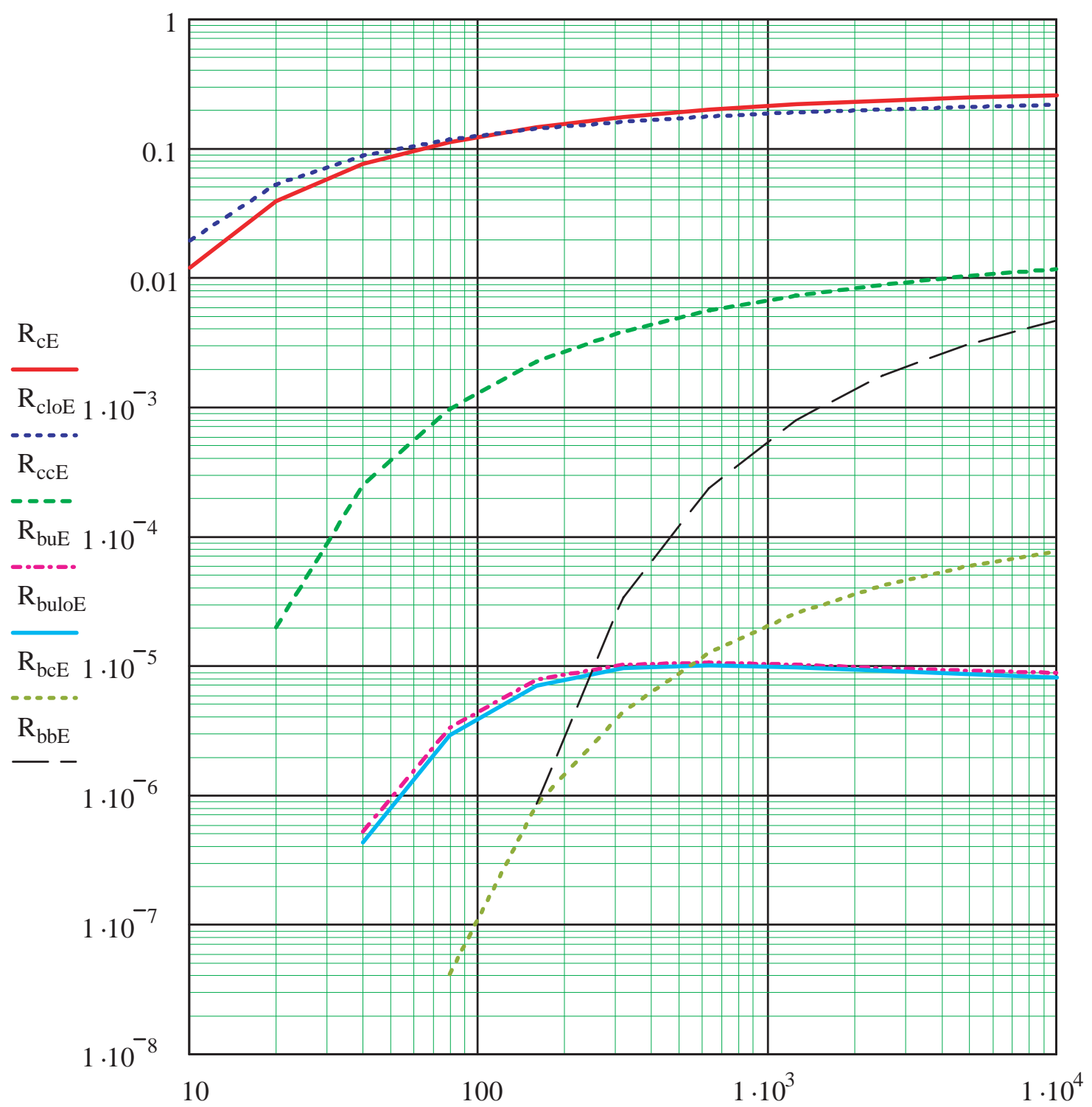

Fig. 5. Heavy quark fractional rates vs neutrino energies corresponding to Fig. 4, but for antineutrinos rather than neutrinos[8].

elements. Other significant contributions to heavy flavor production are proportional to $\left|V_{c s}\right|^{2}$ and $\left|V_{c b}\right|^{2}$, where $\left(V_{c s}, V_{c b}\right)$ are the $(s \rightarrow c, c \rightarrow b)$ CKM elements.

The relatively clean theoretical interpretations and large samples of flavortagged events, particularly for charm production, should allow impressive measurements of the absolute squares for several of the elements in the CKM quark mixing matrix. Estimated precisions in determining the CKM matrix elements are summarized in Table 3. 


\begin{tabular}{|l|lll|}
\hline $\mathbf{u}$ & $\mathbf{d}$ & $\mathbf{s}$ & $\mathbf{b}$ \\
\hline $\mathbf{u}$ & 0.948 & 0.048 & $1.45 \times 10^{-5}$ \\
$\pm 0.16 \%$ & $\pm 2.1 \%$ & $\pm 60 \% \rightarrow O(3 \%)$ \\
& & & \\
& & 1.08 & $1.6 \times 10^{-3}$ \\
& 0.050 & $\pm 31 \% \rightarrow O(3 \%)$ & $\pm 10.5 \% \rightarrow O(3 \%)$ \\
& & & \\
\hline
\end{tabular}

Table 3

Absolute squares of the elements in the first two rows of the Cabbibo-KobayashiMaskawa (CKM) quark mixing matrix, along with their uncertainties when no unitarity constraints are applied [15]. The second row of the entry for each element gives current percentage one-sigma uncertainties in the absolute squares and projections for the uncertainties after analyses from a $\nu \mathrm{MC}$ operating with neutrino energies well above the $B$ production threshold.

Perhaps the most interesting potential measurement outlined in Sec. 3 is the determination of $\left|V_{u b}\right|$ to better than $5 \%$, perhaps eventually reaching $1 \%$. This is an order of magnitude better than the current uncertainty and might well be better than will be achieved in any other single measurement at, for example, a $B$ factory. $B$ production should also allow for an extraction of $\left|V_{c b}\right|$ at the few percent level that is systematically different, in both its experimental and theoretical aspects, from studies of decay processes and that is comparably accurate to the anticipated future measurements using decays.

The matrix element $\left|V_{c d}\right|$ is already best measured from $\mathrm{CC}$ charm production in today's neutrino experiments, based on event samples of several thousands of events. The present accuracy is mainly limited by statistics and uncertainties in charmed hadron production and decay characteristics. It is clear that the accuracy in $\left|V_{c d}\right|$ would be vastly improved from the analysis of hundreds of millions of vertex-tagged charm events in a high performance detector.

\subsubsection{Precision Electroweak Physics}

Section 4 demonstrates that $\nu \mathrm{MCs}$ should be able to provide two types of precision measurements of the weak mixing angle $\sin ^{2} \theta_{W}$ : from the ratio of neutral current (NC) to charged current (CC) DIS events and also from neutrinoelectron scattering.

Both types of determinations require a large extrapolation in event statistics and experimental technique from today's best neutrino results. They will allow vigorous consistency checks of the Standard Model and provide sensitivity to several potential possibilities for new physics. 
With its huge statistics, the DIS measurement of $\sin ^{2} \theta_{W}$ will eventually be systematically limited by theoretical hadronic uncertainties but it should anyway become several times more precise than today's best neutrino measurements, which are already equivalent to about a $100 \mathrm{MeV}$ uncertainty on the $W$ mass.

By contrast, no significant theoretical uncertainties enter into neutrino-electron scattering - a simple scattering process between two elementary point particles - and so the measurements will be limited only by statistics and experimental ingenuity. One can contemplate neutrino-electron scattering event samples as large as $10^{8}$ events using a dedicated detector with parameters like those given in Table 2. This would correspond to impressive statistical uncertainties in $\sin ^{2} \theta_{W}$ of order $10^{-4}$ and sensitivity to new contact interactions at energy scales up to approximately $25 \mathrm{TeV}$. The biggest experimental challenges may come from normalizing the neutrino beam flux. If the experimental uncertainties could be reduced to the extremely challenging level of the statistical uncertainties then this process holds the potential for measurements of $\sin ^{2} \theta_{W}$ that might potentially be as good as or better than the best current measurements from collider experiments.

\subsubsection{Rare and Exotic Processes}

A $\nu \mathrm{MC}$ provides a facility for several unique searches for physics beyond the Standard Model and provides a venue for observing a number of very rare Standard Model processes, as outlined in Sec. 5. Examples of the former include flavor changing $u \rightarrow c, d \rightarrow b$, and $s \rightarrow b$ neutral currents and isosinglet electron and muon-type neutral heavy leptons. A list of the latter includes $\bar{\nu}_{e} e^{-}$annihilation and the scattering of virtual $W$ and $Z$ bosons from quasireal photons in a nuclear Coulomb field; these serve as weak analogs of $e^{+} e^{-}$ and $\gamma \gamma$ physics. Sensitivity to high energy scales through mixing of $W$ or $Z$ with higher mass propagators is limited due to the characteristically weak dependence of the new propagator mass reach as the fourth root of experimental statistics.

\subsubsection{Charm Decays}

A $\nu \mathrm{MC}$ should function as an efficient factory for the study of charm decays, with a clean, well reconstructed sample of several times $10^{8}$ charmed hadrons produced in $10^{10}$ neutrino interactions. Section 6 points out several interesting physics motivations for charm studies at a $\nu \mathrm{MC}$. Measurement of charm decay branching ratios and lifetimes are useful both for QCD studies and for the theoretical calibration of the physics analyses on $B$ hadrons. Charm decays also provide a clean laboratory to search for exotic physics contributions since the Standard Model predicts tiny branching fractions for rare decays, small 
$C P$ asymmetries and slow $D^{0} \rightarrow \overline{D^{0}}$ oscillations.

The charge of the final state lepton in CC-induced charm production from neutrinos tags charm quarks vs. antiquarks with high efficiency and purity. This tag is of particular benefit to oscillation and $C P$ studies, as is the expected precise vertexing reconstruction for the proper lifetime of decays. Section 6 shows it to be quite plausible that a $\nu \mathrm{MC}$ could provide the first observations of both $D^{0}-\bar{D}^{0}$ mixing and $C P$ violation in the charm sector and additionally provide some context for their proper interpretation.

\subsubsection{Neutrino Oscillations}

The potential for long baseline oscillation experiments is the $\nu \mathrm{MC}$ topic that is currently of most interest to the high energy physics community. However, both the experimental and theoretical status of neutrino oscillations are in such a state of flux that long-term predictions for $\nu \mathrm{MCs}$ can be stated only in general terms.

Long baseline experiments at $\nu \mathrm{MCs}$ might well provide a definitive follow-up to the recent intriguing evidence for neutrino oscillations. There will presumably already have been some progress in the verification or refutation of today's oscillation signals by the time long baseline $\nu \mathrm{MCs}$ come on-line. Even so, $\nu \mathrm{MCs}$ will still clearly be important for more probative follow-up studies to characterize the form and phenomenology of any observed oscillation signals. For example, $\nu \mathrm{MCs}$ might help to determine whether an observed oscillation signal is consistent with mixing between three neutrino families or whether a fourth, sterile neutrino is required, as is discussed in Chapter 7. In the former case, long baseline $\nu$ MCs can nail down the values of the mass-squared differences, search for $C P$ violation and look for matter effects in oscillations in the Earth's interior. Clearly, the spectrum of possible studies would be richer still in the case of 4-neutrino mixing.

\section{Deep Inelastic Scattering and QCD Studies}

Starting from SLAC electron scattering experiments in the late 60's, proceeding through the CERN and Fermilab neutrino and muon experiments of the 70's through 90's, and continuing with HERA experiments still underway, deep inelastic scattering (DIS) has provided us with an increasingly accurate

picture of the partonic structure of the nucleon. Moreover, DIS has served and still serves as one of the best test-beds for perturbative QCD.

A $\nu \mathrm{MC}$ could take the physics of DIS to a new level by: (1) providing the 
statistical power to extract all six structure functions for $\nu$ and $\bar{\nu}$ beams on proton and deuterium targets; (2) allowing for low mass, high acceptance spectrometers with vastly improved resolution over present calorimetric detectors; (3) creating naturally redundant measurements through simultaneous measurement of electron and muon final scattering final states; (4) generating the rate and small beam spot size required for the first polarized neutrino targets; (5) permitting use of active vertexing targets for systematic studies of heavy flavor production, and (6) facilitating the use of a large array of nuclear targets.

Examples of physics topics that would emerge include:

- Definitive proton parton distribution functions (PDF) for $x \geq 0.01$. The $x \geq 0.01$ behavior of parton densities at accessible $\nu \mathrm{MC} Q^{2}$ controls the cross section behavior of the highest energy scale physics of the Tevatron and LHC. Understanding subtle deviations caused by new physics requires precise control of PDF systematics. A $\nu \mathrm{MC}$ will have the statistical power and the systematic redundancy checks to generate a complete PDF set from a single experiment.

- A test of $Q C D$ to next-to-next to leading order (NNLO). Few experiments quantitatively test QCD beyond leading order in the coupling $\alpha_{s}\left(Q^{2}\right)$. It is frequently the case instead that leading order (LO) and next-to-leading order (NLO) provide equally good descriptions of the data; and the NLO calculation is preferred mainly because it reduces theoretical systematic uncertainties in quantities such as $\alpha_{s}\left(Q^{2}\right)$. As an inclusive scattering process at space-like momentum transfer, DIS is perhaps the phenomenon for which QCD is most rigorously applicable. NLO cross sections are already fully calculated and NNLO computations for several processes exist as well. Testing the entire theory at NNLO seems feasible.

- Precise measurements of $\alpha_{s}$. at moderate $Q^{2}$ The running of the strong coupling constant is largely determined by excellent measurements of the $\tau$ lepton hadronic decay width at $s=m_{\tau}^{2}$ as well as a series of precise measurements at $s=M_{Z}^{2}$. Getting this running right is important; for example, one of the few experimental pieces of evidence for supersymmetry is its success in getting the strong, weak, and electromagnetic couplings to unify at one scale. Given this importance, further precise measurements of $\alpha_{s}$ at scales between $m_{\tau}^{2}$ and $m_{Z}^{2}$ are valuable. A $\nu \mathrm{MC}$ provides at least two ways of achieving these: through scaling violations in non-singlet structure functions and through evaluations of the Gross-Lleweyllyn-Smith sum rule.

- Studies of two-scale QCD via $\nu$ and $\bar{\nu}$ heavy flavor production. A quark $q$ is treated as a heavy object in DIS if $Q^{2}<m_{q}^{2}$ whereas it is instead considered to be a parton when $\log \left(Q^{2} / m_{q}^{2}\right) \gg 1$. Deep inelastic scattering at a $\nu \mathrm{MC}$ allows study of the transition of $q$ from heavy quark to parton by opening the possibility of measuring quark mass effects at a series of scales in CC and NC scattering. The possibility of using vertexing targets maintains the 
inclusive nature of measurements by avoiding the need for final state lepton tagging.

- Neutrino Spin Physics. Charged lepton scattering experiments from polarized targets show that $u$ and $d$ type quarks carry very little of the nucleon spin and have hinted at strong polarization effects in gluons and strange quarks. A $\nu \mathrm{MC}$ creates the first possibility of using polarized targets for neutrinos and brings all of their power for flavor and helicity selection to bear on nucleon spin physics.

- Neutrino Nuclear Physics. Thin nuclear targets at a $\nu \mathrm{MC}$ can rapidly acquire the statistics to make measurements of the $A$ dependence of the $F_{2}$ structure function for neutrinos that complement those from charged lepton scattering. The first precise measurements of the $A$ dependence of $x F_{3}$ will become available as well.

\subsection{Background on Measuring Parton Distribution Functions and QCD with Non-Polarized Targets}

Invariance principles dictate the general form of $\nu N(N=p$ or $n)$ nucleon scattering. For energies much greater than the final state lepton mass and to leading order in electroweak couplings:

$$
\begin{aligned}
\frac{d^{2} \sigma_{C C / N C}^{\nu N(\bar{\nu} N)}}{d x d y} & =\frac{G_{F}^{2} M_{N} E_{\nu}}{\pi\left(1+Q^{2} / M_{V}^{2}\right)^{2}}\left[\left(1-y-\frac{M_{N} x y}{2 E_{\nu}}\right) F_{2, C C / N C}^{\nu N(\bar{\nu} N)}\left(x, Q^{2}\right)\right. \\
& \left.+\left(\frac{y^{2}}{2}\right) 2 x F_{1, C C / N C}^{\nu N(\bar{\nu} N)}\left(x, Q^{2}\right) \pm y(1-y / 2) x F_{3, C C / N C}^{\nu N(\bar{\nu} N)}\left(x, Q^{2}\right)\right]
\end{aligned}
$$

with $G_{F}$ the Fermi coupling constant, $M_{N}$ the nucleon mass, $E_{\nu}$ the neutrino energy, $y$ the inelasticity, $x$ the Bjorken scaling variable, and $Q^{2}$ the negative squared four-momentum transfer to the nucleon target. The plus (minus) sign in the final term is conventional for neutrino (antineutrino) scattering, and $M_{V}=M_{W}\left(M_{Z}\right)$ for $\mathrm{CC}(\mathrm{NC})$ scattering. The structure functions $2 x F_{1, C C / N C}^{\nu N(\bar{\nu} N)}\left(x, Q^{2}\right), F_{2, C C / N C}^{\nu N(\bar{\nu} N)}\left(x, Q^{2}\right)$ and $x F_{3, C C / N C}^{\nu N(\bar{\nu} N)}\left(x, Q^{2}\right)$ contain all the information about the internal structure of the target. The cross sections for electron-neutrinos and muon-neutrinos are nearly identical, up to electroweak radiative corrections.

The SF depend on the $A$ and $Z$ of the target nucleus, on whether the beam is neutrino or antineutrino, and on whether the scattering is $\mathrm{CC}$ or NC. They can be experimentally extracted in principle by measuring the differential cross sections in fixed $x$ and $Q^{2}$ bins as a function of $y$ and then exploiting the $y$ dependences shown in Eq. 10 to fit for $2 x F_{1}, F_{2}$, and $x F_{3}$. In practice, the reduced $y$ coverage created by cuts on final state lepton energies and kinematic constraints limits this procedure, and various model-dependent alternatives 
have been assumed. For example, the SF $2 x F_{1}$ has rarely been measured in neutrino scattering; instead it has been related to $F_{2}$ through a model for the longitudinal structure function

$$
R_{L}\left(x, Q^{2}\right) \equiv \frac{F_{2}\left(x, Q^{2}\right)\left(1+4 M^{2} x^{2} / Q^{2}\right)}{2 x F_{1}\left(x, Q^{2}\right)}-1,
$$

with $R_{L}\left(x, Q^{2}\right)$ computed from QCD or taken from charged lepton scattering. Charged current interactions, with their observable lepton in the final state, can be much better reconstructed than NC interactions and so we will assume $\mathrm{CC} \mathrm{SF}$ in the discussion that follows.

Neutrino-nucleon scattering is the only DIS process that can provide measurements of the parity-violating $F_{3}$ structure functions, apart from the much less precise measurements in a different kinematic regime from HERA. The parity-conserving $2 x F_{1}$ and $F_{2}$ structure functions for neutrino-nucleon scattering probe different combinations of quarks to the analogous SFs defined for charged lepton DIS experiments.

A rough summary of the current knowledge of neutrino SF follows. A more complete review may be found in Ref. 12 .

(1) Measurements at an accuracy of a few percent exist for $n / p, \nu / \bar{\nu}$ averaged $\mathrm{CC} \mathrm{SF}$

$$
\begin{aligned}
F_{2, C C}\left(x, Q^{2}\right) & =\frac{1}{4} \sum_{k=\nu, \bar{\nu}} \sum_{N=n, p} F_{2, C C}^{k N}\left(x, Q^{2}\right), \\
x F_{3, C C}\left(x, Q^{2}\right) & =\frac{1}{4} \sum_{k=\nu, \bar{\nu}} \sum_{N=n, p} x F_{3, C C}^{k N}\left(x, Q^{2}\right),
\end{aligned}
$$

using iron targets for $10^{-3} \lesssim x \lesssim 0.7$ and $Q^{2} \lesssim 200 \mathrm{GeV}^{2}$. The $x$ and $Q^{2}$ ranges are highly correlated by the limited range of beam energies. These measurements assume a model for $R_{L}\left(x, Q^{2}\right)$. Uncertainties on $F_{2, C C}\left(x, Q^{2}\right)$ are dominated by systematic effects, while $x F_{3, C C}\left(x, Q^{2}\right)$ errors still contain a significant statistical contribution.

(2) Measurements at the $\sim 10 \%$ level exist for:

(a) the $n / p, \nu / \bar{\nu}$ averaged $R_{L}\left(x, Q^{2}\right)$ using an iron target;

(b) the $n / p$ averaged $x F_{3, C C}^{\nu}\left(x, Q^{2}\right)-x F_{3, C C}^{\bar{\nu}}\left(x, Q^{2}\right)$;

(c) $F_{2, C C}^{k p}\left(x, Q^{2}\right), F_{2, C C}^{k D}\left(x, Q^{2}\right)$ and $x F_{3, C C}^{k p}, x F_{3, C C}^{k D}$ for $k=\nu, \bar{\nu}$ and with $D=$ deuterium.

(3) No SF-oriented neutrino experiments are currently in operation and no new experiments are planned other than a possible $\nu \mathrm{MC}$ program.

The SF goal for an $\nu \mathrm{MC}$ is simple: to measure, over as wide a range of $x$ and $Q^{2}$ as possible, the six SF of Eq. 10, particularly for the proton and deuteron but also for other nuclear targets. 


\subsection{Measurement of Quark Parton Distribution Functions}

Structure functions provide much of the information used to deduce PDFs. In turn, the PDFs are crucial for all predictions of event rates at the Tevatron and LHC. To avoid nuclear complications, $\nu \mathrm{MC}$ SF should be extracted with proton and deuterium targets.

To leading order (LO) in QCD, the SF can be expressed in terms of nucleon $\mathrm{PDF}$ as [2:

$$
\begin{aligned}
F_{2, C C}^{\nu N}\left(x, Q^{2}\right) & =2\left[d^{N}\left(x, Q^{2}\right)+s^{N}\left(x, Q^{2}\right)+\bar{u}^{N}\left(x, Q^{2}\right)+\bar{c}^{N}\left(x, Q^{2}\right)\right], \\
F_{2}^{\bar{\nu} N}\left(x, Q^{2}\right) & =2\left[u^{N}\left(x, Q^{2}\right)+c^{N}\left(x, Q^{2}\right)+\bar{d}^{N}\left(x, Q^{2}\right)+\bar{s}^{N}\left(x, Q^{2}\right)\right], \\
x F_{3}^{\nu N}\left(x, Q^{2}\right) & =2\left[d^{N}\left(x, Q^{2}\right)+s^{N}\left(x, Q^{2}\right)-\bar{u}^{N}\left(x, Q^{2}\right)-\bar{c}^{N}\left(x, Q^{2}\right)\right], \\
x F_{3}^{\bar{\nu} N}\left(x, Q^{2}\right) & =2\left[u^{N}\left(x, Q^{2}\right)+c^{N}\left(x, Q^{2}\right)-\bar{d}^{N}\left(x, Q^{2}\right)-\bar{s}^{N}\left(x, Q^{2}\right)\right] ;
\end{aligned}
$$

and $x$ can be identified as the target's fractional 4-momentum carried by the struck quark. The Callan-Gross relation holds,

$$
2 x F_{1}^{\nu N(\bar{\nu} N)}\left(x, Q^{2}\right)=F_{2}^{\nu N(\bar{\nu} N)}\left(x, Q^{2}\right),
$$

implying that $F_{2}^{\nu N(\bar{\nu} N)}\left(x, Q^{2}\right)$ and $2 x F_{1}^{\nu N(\bar{\nu} N)}\left(x, Q^{2}\right)$ provide redundant parton information.

Measurements of the eight independent observables of Eq. 13 (two SF for each beam on two targets) represents more information than is available from charged lepton scattering but is not enough to specify the eighteen independent PDF for each target $N(u, d, s, c$, plus their antiquarks and the gluon

2 We adopt the convention that the PDF are given as parton probability functions multiplied by $x$, i.e. $u\left(x, Q^{2}\right)$ is $x$ times the probability of finding a $u$ quark with momentum fraction $x$. This is close to what is actually measured in experiments and is also the form provided in compilations such as PDFLIB[16]. 
for $n$ and $p$ ). Further constraints emerge from isospin symmetry:

$$
\begin{aligned}
u^{n}\left(x, Q^{2}\right) & =d^{p}\left(x, Q^{2}\right) \equiv d\left(x, Q^{2}\right), \\
d^{n}\left(x, Q^{2}\right) & =u^{p}\left(x, Q^{2}\right) \equiv u\left(x, Q^{2}\right), \\
\bar{u}^{n}\left(x, Q^{2}\right) & =\bar{d}^{p}\left(x, Q^{2}\right) \equiv \bar{d}\left(x, Q^{2}\right), \\
\bar{d}^{n}\left(x, Q^{2}\right) & =\bar{u}^{p}\left(x, Q^{2}\right) \equiv \bar{u}\left(x, Q^{2}\right), \\
s^{n}\left(x, Q^{2}\right) & =s^{p}\left(x, Q^{2}\right) \equiv s\left(x, Q^{2}\right), \\
\bar{s}^{n}\left(x, Q^{2}\right) & =\bar{s}^{p}\left(x, Q^{2}\right) \equiv \bar{s}\left(x, Q^{2}\right), \\
c^{n}\left(x, Q^{2}\right) & =c^{p}\left(x, Q^{2}\right) \equiv c^{p}\left(x, Q^{2}\right), \\
\bar{c}^{n}\left(x, Q^{2}\right) & =\bar{c}^{p}\left(x, Q^{2}\right) \equiv \bar{c}\left(x, Q^{2}\right), \\
g^{n}\left(x, Q^{2}\right) & =g^{p}\left(x, Q^{2}\right) \equiv g\left(x, Q^{2}\right) .
\end{aligned}
$$

The nine extra constraints of Eqs. 15 reduce the number of independent PDF to nine. More reduction occurs if one assumes

$$
\bar{s}\left(x, Q^{2}\right)=s\left(x, Q^{2}\right),
$$

which is supported by measurements of dimuon production in $\nu F e$ and $\bar{\nu} F e$ scattering $[17,18]$. Furthermore, at the $Q^{2}$ accessible to lower energy $\nu \mathrm{MCs}$, one can consistently adopt a three-flavor QCD scheme, whence

$$
c\left(x, Q^{2}\right)=\bar{c}\left(x, Q^{2}\right)=0
$$

these relations are supported by measurements of $\mathrm{NC}$ charm production in $\nu_{\mu} F e$ and $\bar{\nu}_{\mu} F e$ scattering $[19,20]$. This finally leaves six independent PDF: $\bar{u}\left(x, Q^{2}\right), \bar{d}\left(x, Q^{2}\right), s\left(x, Q^{2}\right), g\left(x, Q^{2}\right)$, and the valence distributions

$$
\begin{aligned}
& u_{V}\left(x, Q^{2}\right)=u\left(x, Q^{2}\right)-\bar{u}\left(x, Q^{2}\right), \\
& d_{V}\left(x, Q^{2}\right)=d\left(x, Q^{2}\right)-\bar{d}\left(x, Q^{2}\right) .
\end{aligned}
$$

At LO, the four deuterium SF can be combined to yield the total quark and valence quark distributions

$$
\begin{aligned}
F_{2, C C}^{\nu D}\left(x, Q^{2}\right) & =F_{2, C C}^{\bar{\nu}}\left(x, Q^{2}\right) \\
& =u\left(x, Q^{2}\right)+d\left(x, Q^{2}\right) \\
& +\bar{u}\left(x, Q^{2}\right)+\bar{d}\left(x, Q^{2}\right)+2 s\left(x, Q^{2}\right), \\
\frac{1}{2}\left[x F_{3, C C}^{\nu D}\left(x, Q^{2}\right)+F_{3, C C}^{\bar{\nu} D}\left(x, Q^{2}\right)\right] & =u_{V}\left(x, Q^{2}\right)+d_{V}\left(x, Q^{2}\right) .
\end{aligned}
$$

The four individual proton SF then allow separation of the $u$ and $d$ quark contributions. Sensitivity to $s\left(x, Q^{2}\right)$ emerges from

$$
x F_{3, C C}^{\nu D}\left(x, Q^{2}\right)-x F_{3, C C}^{\bar{\nu} D}\left(x, Q^{2}\right)=4 s\left(x, Q^{2}\right),
$$


and, in principle, from comparison to the charged lepton $F_{2, C C}^{\ell D}\left(x, Q^{2}\right)$,

$$
\frac{5}{18} F_{2, C C}^{\nu D}\left(x, Q^{2}\right)-F_{2}^{\ell D}\left(x, Q^{2}\right)=\frac{1}{3} s\left(x, Q^{2}\right) .
$$

Practical implementation of Eqs. 20 and 21 has been stymied by difficulties in controlling systematic errors, and current measurements of $s\left(x, Q^{2}\right)$ all come from semi-inclusive $\nu_{\mu} N$ and $\bar{\nu}_{\mu} N$ charm production.

The gluon PDF does not enter directly at LO for CC SF. It affects the QCD evolution of $F_{2}$ and $2 x F_{1}$, appears with next-to-leading order (NLO) cross section terms, and enters directly into semi-inclusive double heavy quark production through the $\mathrm{NC}$ and $\mathrm{CC}$ processes

$$
\begin{aligned}
& \nu_{\ell} N \rightarrow \nu_{\ell} c \bar{c} X \\
& \nu_{\ell} N \rightarrow \nu_{\ell} b \bar{b} X \\
& \nu_{\ell} N \rightarrow \ell^{-} c \bar{b} X
\end{aligned}
$$

as well as neutrino $J / \psi$ and $\Upsilon$ production[21].

\subsection{Tests of Perturbative QCD}

At a $\nu \mathrm{MC}$, data will be of sufficient precision to probe QCD to NLO, and perhaps to NNLO.

In practice, the data will be in the form of differential cross sections in $x$ and $y$ at different neutrino energies. The general procedure for a QCD analysis of the data consists of: (1) defining a favorable kinematic region where pQCD is expected to apply to within small corrections; (2) choosing a parameterization

of the six PDF $\vec{p}\left(x, Q_{0}^{2} ;\{\lambda\}\right)$, where $\vec{p}=\left(u_{V}, d_{V}, \bar{u}, \bar{d}, \bar{s}, g\right), Q_{0}^{2}$ is a reference $Q^{2}$ and $\{\lambda\}$ are a set of parameters describing the PDF; (3) using pQCD to fit the data over all $Q^{2}$ by varying $\{\lambda\}$.

The quality of such a fit to the data constitutes an immediate test of QCD. For example, QCD can be verified to NLO if NLO pQCD provides a better fit than LO. While contemporary practice dictates fitting with NLO pQCD, it is worth pointing out that neutrino data do not convincingly favor the higher order calculation over a LO interpretation. Assuming a good pQCD fit, one can then go on to extract the single parameter of QCD itself, which can taken to be the strong coupling constant evaluated at the reference $Q^{2}$ of the experiment $\alpha_{s}\left(Q_{0}^{2}\right)$.

As a technical comment, the fit procedure is admittedly somewhat complex, and the need to treat PDF parameterizations and QCD together arises from 
kinematic acceptance issues. The $Q^{2}$ value will anyway be limited to $Q^{2} \lesssim$ $2 M E$ and cuts on final state lepton energies may further limit the available $\left(x, Q^{2}, y\right)$ phase space, particularly at lower energy $\nu$ MCs. More precisely, if $P_{\ell}^{\min }$ and $E_{H A D}^{\min }$ represent the minimum acceptable final state lepton and hadron energies, then for a given $Q^{2}$, one has

$$
\begin{aligned}
& Q^{2} / 2 M\left(E-P_{\ell}^{\min }\right) \lesssim x \\
& E_{H A D}^{\min } / E \lesssim y \\
& \lesssim 1-P_{\ell}^{\min } / E
\end{aligned}
$$

This implies that it is impossible to span all of $x$ for a fixed $Q^{2}$ in order to extract the PDF of Eqs. 13. An interpolation scheme is needed to connect different regions of $x$ and $Q^{2}$ space; fortunately, pQCD provides just that scheme through the DGLAP equations[22-25].

More important even than the high statistics and potentially improved systematics promised by $\nu \mathrm{MCs}$ are the richness of evolution tests created by the availability of 12 proton and deuterium SF, even with the limited phase space. Examples include:

(1) $\frac{1}{2}\left[x F_{3, C C}^{\nu D}\left(x, Q^{2}\right)+x F_{3, C C}^{\bar{\nu} D}\left(x, Q^{2}\right)\right]$ : the "classic" non-singlet SF's evolution is independent of $g\left(x, Q^{2}\right)$ but suffers uncertainties from charm production.

(2) $\frac{1}{2}\left[F_{2, C C}^{\nu D}\left(x, Q^{2}\right)+F_{2, C C}^{\bar{\nu} D}\left(x, Q^{2}\right)\right]$ : the most precisely measurable SF usefully constrains $g\left(x, Q^{2}\right)$ and cross-checks charged lepton scattering.

(3) $F_{2, C C}^{\bar{\nu} D}\left(x, Q^{2}\right)-F_{2, C C}^{\bar{\nu} p}\left(x, Q^{2}\right)$ : a new combination for a $\nu \mathrm{MC}$; this difference is both independent of $g\left(x, Q^{2}\right)$ and charm production.

Sum rule-tests comprise some of the most accurately calculated observables in QCD. For example, the Gross Llewellyn Smith (GLS) sum rule [26,28] yields:

$$
\begin{aligned}
S_{G L S}\left(Q^{2}\right) & \equiv \int_{0}^{1} \frac{d x}{x}\left[x F_{3, C C}^{\nu D}\left(x, Q^{2}\right)+x F_{3, C C}^{\nu D}\left(x, Q^{2}\right)\right] d x \\
& =3\left[1-\frac{\alpha_{s}}{\pi}-a\left(N_{f}\right)\left(\frac{\alpha_{s}}{\pi}\right)^{2}\right. \\
& \left.-b\left(N_{f}\right)\left(\frac{\alpha_{s}}{\pi}\right)^{3}+O\left(\frac{\alpha_{s}^{4}}{\pi^{4}}\right)+O^{\prime}\left(\frac{M^{2}}{Q^{2}}\right)\right],
\end{aligned}
$$

with $a\left(N_{f}\right)$ and $b\left(N_{f}\right)$ are known functions of $Q^{2}$ and the specified number of active flavors $N_{f}$ used in the pQCD analysis. Corrections from order $\left(\alpha_{S} / \pi\right)^{4}$ pQCD [27] and order $M^{2} / Q^{2}$ higher twist effects have been calculated[29,30]. A $\nu \mathrm{MC}$ would further provide the targets and the statistics to evaluate for the first time with a precision sufficient to test QCD through the Adler sum[31]

$$
S_{A}\left(Q^{2}\right)=2 \int_{0}^{1} \frac{d x}{x}\left[F_{2, C C}^{\nu D}\left(x, Q^{2}\right)-F_{2, C C}^{\nu p}\left(x, Q^{2}\right)\right] d x
$$


assuming that $2 F_{2, C C}^{\nu D}\left(x, Q^{2}\right)=F_{2, C C}^{\nu p}\left(x, Q^{2}\right)+F_{2, C C}^{\nu n}\left(x, Q^{2}\right)$.

Structure function analyses in neutrino scattering are a complex business that requires a painstaking attention to systematic error sources. The current best QCD measurements - the evolution[32] of $x F_{3}$ and the GLS sum rule[33] - are limited by energy calibration uncertainties in the former and flux-related errors in the latter. Calibration uncertainties will be reduced at $\nu \mathrm{MCs}$ through use of lower mass particle spectrometers that allow better resolution and the possibility of using in situ sources such as $K_{S}^{0}$ and $J / \psi$ decay. The ability to simultaneously measure $\bar{\nu}_{e}$ and $\nu_{\mu}$ scattering provides built in cross-checks, and the $\bar{\nu}_{e}$ CC scattered electrons can be measured both magnetically and calorimetrically to cross-calibrate the spectrometer and calorimeter. Flux errors will be diminished considerably by the simplicity of the neutrino source compared to $\pi / K$ decay beams, which permits much more reliable monitoring. High rates will also allow direct flux measurement in the $\nu_{\mu} \bar{\nu}_{e}$ mode through use of the electroweak reactions $\nu_{\mu} e^{-} \rightarrow \mu^{-} \nu_{e}$ and $\bar{\nu}_{e} e^{-} \rightarrow \mu^{-} \bar{\nu}_{\mu}$.

\subsection{Heavy Quark Production}

The simple language of the quark parton model must be modified for $\nu N$ DIS events with a charm or beauty quark in the final state in order to take into account the non-negligible quark mass. This presents both a challenge and an opportunity to test the QCD formalism for making these corrections.

Perhaps the simplest and most widely used correction scheme is the essentially kinematic "slow rescaling" model of Georgi and Politzer [34], which amounts to a redefinition of the scaling variable $x$ through

$$
x \rightarrow \xi=x\left(1+\frac{m_{Q}^{2}}{Q^{2}}\right),
$$

where $m_{Q}$ is a heavy quark mass. One of the shortcomings of this prescription is that it fails to address ambiguities in the PDF that arise when one attempts a self-consistent pQCD treatment of heavy quark production.

Neutral current charm production illustrates this ambiguity. On the one hand, this can be treated as a "flavor-creation" process, $Z+g \rightarrow c \bar{c}$, which occurs at order $\alpha_{s}^{1}$ in the pQCD expansion. In this case, the appropriate scheme for calculation of QCD radiative corrections involves only three light flavors (the so-called three-flavor scheme), and all of the effects associated with the charm quark are accounted for perturbatively in the hard scattering. On the other hand, the charm quark can be considered to be "light" at high energies when $Q^{2} \gg m_{c}^{2}$ so the mass of the charm quark is much smaller than the relevant physical scale. In this case, one can view the charm quark as a parton with a 
corresponding parton distribution function (the so-called four-flavor scheme). In particular, the lowest order Wilson coefficient for charm production in this situation is a "flavor-excitation" process, $Z+c \rightarrow c$, which is of order $\alpha_{s}^{0}$. These two viewpoints must be reconciled in a consistent manner in order to avoid double counting.

The corresponding calculational problem for pQCD is the presence of a heavy quark mass scale that can be comparable to the $Q^{2}$ of the interaction. In the absence of this complication, the factorization theorem of QCD separates the high scale set by the value of transferred momentum, $\mu_{H}^{2} \sim Q^{2}$, from the low scale of hadronic physics, $\mu_{L}^{2} \sim \Lambda_{Q C D}^{2}$, and operator product expansion techniques can be used to sum the large logarithms of the form $\log \left(\mu_{h}^{2} / \mu_{l}^{2}\right)$ that multiply the expansion parameter, $\alpha_{s}$.

A practical recipe for incorporating heavy quark masses in the pQCD summations is provided by the ACOT [35] prescription, which treats the number of active flavors, $N_{f}$, as a scale-dependent quantity. In particular, it suggests the use of a three-flavor evolution for $\mu<m_{c}$ and a four-flavor evolution above $m_{c}$, with continuity at the break point. In this prescription, the parton distribution functions are labeled by the number of active flavors and the heavy quark parton distribution functions, $f_{Q / N}(\xi, \mu)$, vanish for $\mu_{H} \leq m_{Q}$ and satisfy the usual $\bar{M} S$ QCD evolution equation (with massless kernel functions) for $\mu_{h}>m_{Q}$.

Charm and beauty production at $\nu$ MCs will include large numbers of events with $Q^{2}$ above, below and around the effective $m_{Q}^{2}$ scale and should provide the most stringent tests from any experimental process of the pQCD formalism for heavy quark production. At that point, the experimental precision will likely require a more careful quantum field theoretical treatment of heavy quark masses.

Available rates at a $\nu \mathrm{MC}$ (Figs. 4 and 5) provide access to five different values for $m_{Q}: m_{c}\left(\right.$ from $\left.\nu_{\ell} N \rightarrow \ell^{-} c X\right), 2 m_{c}\left(\right.$ from $\left.\nu_{\ell} N \rightarrow \nu_{\ell} c \bar{c} X\right), m_{b}\left(\right.$ from $\nu_{\ell} N \rightarrow$ $\left.\ell^{-} \bar{b} X\right), m_{b}+m_{c}\left(\right.$ from $\left.\nu_{\ell} N \rightarrow \ell^{-} c \bar{b} X\right)$, and $2 m_{b}$ (from $\left.\nu_{\ell} N \rightarrow \nu_{\ell} b \bar{b} X\right)$. To the degree a consistent pair of values for $m_{b}$ and $m_{c}$ emerges, one would have established theoretical control over these production processes. Those mass values could also be compared to what QCD sum rules, heavy quark expansions and lattice QCD yield. They can also be used to calibrate models for heavy quark production that enter into other $\nu \mathrm{MC}$ analyses, such as the pQCD tests described above and precision $\sin ^{2} \theta_{W}$ measurements in inclusive NC scattering discussed in Sec. 4.

Other than the ubiquitous high rates, the main experimental asset for a $\nu \mathrm{MC}$ in these studies will be the opportunity to use vertexing targets. This should allow for lifetime tagging of heavy flavors that minimizes systematic effects 
due to fragmentation and decay uncertainties.

\subsection{Parton Distribution Functions at Large Bjorken $x$}

The unique level of quark-by-quark characterization of nucleon structure expected at $\nu \mathrm{MCs}$ will provide an invaluable reference source for many diverse analyses in collider and fixed target physics including, of course, other precision analyses at $\nu$ MCs. Precise measurements as $x \rightarrow 1$ are particularly relevant to the modeling of rates for interesting physics processes and backgrounds at hadron colliders because uncertainties at high $x$ and at the typical $Q^{2}$ values for $\nu \mathrm{MCs}$ will evolve to uncertainties at much lower $x$ as $Q^{2}$ increases to collider values. Uncertainties at high $x$ in current nucleon PDF derive from two sources: the ratio $d\left(x, Q^{2}\right) / u\left(x, Q^{2}\right)$ as $x \rightarrow 1$ and the role of higher twist corrections.

Analyses on present leptoproduction data sets that used hydrogen and deuterium targets have been unable to precisely pin down the high- $x$ behavior of $d(x) / u(x)$. For example, QCD fits [36] to the high- $x$ NMC data and the CDF $W$-decay asymmetry improve with the inclusion of a simple $x$-dependent correction for a $d(x) / u(x)$ ratio that asymptotically approaches 0.2 rather than zero for $x \rightarrow 1$. On the other hand, the recent CTEQ5 global QCD analysis [37] found that including this correction had little effect on the quality of their fits.

Besides the statistical and experimental uncertainties in the data sets, a complication with high $x$ analyses is the need to model nuclear binding effects in deuterium This issue can be avoided at a $\nu \mathrm{MC}$, where a high statistics exposure to a $H_{2}$ target alone could directly measure the $d(x) / u(x)$ ratio in protons as $x \rightarrow 1$ from the ratio of $\nu_{\ell} p$ to $\bar{\nu}_{\ell} p$ cross sections. Such a measurement would require only a small correction for the residual sea quark contributions at high $x$.

Measurement of quark PDFs at high $x$ is closely related to the question of the leading power corrections in the QCD perturbative expansion that are known as "higher twist effects". The $n^{\text {th }}$ order higher twist effects are proportional to $1 / Q^{2 n}$ and reflect the fact that quarks have transverse momentum within the nucleon and that the probe becomes larger as $Q^{2}$ decreases, thus increasing the probability of multi-quark participation in an interaction. As was the case with the $u / d$ ratio, different analyses of higher twist corrections in current data leave unresolved issues that would benefit from new experimental information.

An analysis by Milsztajn[38] that combined electroproduction data from SLAC with BCDMS muo-production data found that the relative size of the twist4 contribution rapidly increased above $x=0.4$ and was equal in magnitude 
to the leading $1 / \log Q^{2}$ term for $x>0.75$. The only measurements of this higher-twist term in neutrino experiments have been two low-statistics bubble chamber experiments: in Gargamelle [39] with freon and in BEBC with $\mathrm{NeH}_{2}$. Both bubble chamber analyses are complicated by nuclear corrections at high$x$. However, both found a twist-4 contribution that is smaller in magnitude and, most significantly, of opposite sign to that of the charged leptoproduction analysis.

In contrast, a CTEQ global QCD analysis that combines neutrino and charged lepton production analyses up to $x=0.75$, finds that no higher-twist term is required for values of $Q^{2}$ down to $0.7 \mathrm{GeV}^{2}$. However, this analysis uses a cut on the invariant mass of the hadronic system, $W>4 \mathrm{GeV}$, that could exclude the bulk of any twist- 4 contribution. Most recently, the Yang-Bodek analysis mentioned above [36] reanalyzed electroproduction data looking for a higher twist contribution. They find that by incorporating a NLO QCD analysis, as opposed to the LO analysis used by Milsztajn, the higher twist contribution becomes much smaller. However, they have also included the $d / u \rightarrow 0.2$ model in their extraction of higher twist, which will reduce the size of any extracted higher twist term.

From a more theoretical viewpoint, a recent CTEQ paper by Guo and Qiu [40] has predicted the leading $x$ dependence to the higher-twist term in $F_{2}\left(x, Q^{2}\right)$ in the region of large $x$ and found that the higher-twist contribution is different for $u$ and $d$ quarks. Were this to be true, then the $d(x) / u(x)$ analysis and the leading power correction analysis would be directly intertwined. Interestingly enough, this prediction only depends on two non-perturbative parameters and is therefore highly constrained. It could be tested at both CEBAF and a $\nu \mathrm{MC}$.

\subsection{Examining the Spin Structure of the Nucleon}

A unique new feature of $\nu$ MCs would be the availability of sufficiently intense beams to allow, for the first time, measurements of neutrino scattering off nucleons in polarized targets. This would provide access to the polarized nucleon structure functions for neutrino-nucleon scattering and could answer several currently unresolved questions about the spin structure of the nucleon.

Polarized structure functions (PSFs) can be represented approximately by the quark-parton model in terms of the differences between the parton densities

of quarks polarized parallel to the nuclear spin and those that are polarized 
anti-parallel:

$$
\begin{aligned}
& \delta q\left(x, Q^{2}\right)=q^{\uparrow \uparrow}\left(x, Q^{2}\right)-q^{\uparrow \downarrow}\left(x, Q^{2}\right), \\
& \delta \bar{q}\left(x, Q^{2}\right)=\bar{q}^{\uparrow \uparrow}\left(x, Q^{2}\right)-\bar{q}^{\uparrow \downarrow}\left(x, Q^{2}\right) \cdot \delta \bar{g}\left(x, Q^{2}\right)=\bar{g}^{\uparrow \uparrow}\left(x, Q^{2}\right)-\bar{g}^{\uparrow \downarrow}\left(x, Q^{2}\right) .
\end{aligned}
$$

In the naive (LO) quark-parton model, the polarized structure functions $g_{1}$ and $g_{3}$ have quark spin content corresponding to the quark content of the parity conserving and parity violating unpolarized structure functions $F_{1}$ and $F_{3}$, respectively:

$$
\begin{aligned}
& g_{1}^{\nu N}\left(x, Q^{2}\right)=\delta d^{N}\left(x, Q^{2}\right)+\delta s^{N}\left(x, Q^{2}\right)+\delta \bar{u}^{N}\left(x, Q^{2}\right)+\delta \bar{c}^{N}\left(x, Q^{2}\right), \\
& g_{1}^{\bar{\nu} N}\left(x, Q^{2}\right)=\delta u^{N}\left(x, Q^{2}\right)+\delta c^{N}\left(x, Q^{2}\right)+\delta \bar{d}^{N}\left(x, Q^{2}\right)+\delta \bar{s}^{N}\left(x, Q^{2}\right),
\end{aligned}
$$

and

$$
\begin{aligned}
& 2 x g_{3}^{\nu p}\left(x, Q^{2}\right)=-\left[\delta d\left(x, Q^{2}\right)+\delta s\left(x, Q^{2}\right)-\delta \bar{u}\left(x, Q^{2}\right)-\delta \bar{c}\left(x, Q^{2}\right)\right] \\
& 2 x g_{3}^{\bar{\nu} p}\left(x, Q^{2}\right)=-\left[\delta u\left(x, Q^{2}\right)+\delta c\left(x, Q^{2}\right)-\delta \bar{d}\left(x, Q^{2}\right)-\delta \bar{s}\left(x, Q^{2}\right)\right] .
\end{aligned}
$$

The other parity-conserving PSF, $g_{2}$, has no simple interpretation in the LO quark-parton model, as is also the case with the parity-violating $g_{4}$. The remaining PSF, $g_{5}$, is related to $g_{3}$ via $g_{5}=2 x g_{3}$ in the naive quark-parton model.

The levels of the different polarized quark PDF's can be regarded as quantifying the extent to which a parton of flavor $q$ "remembers" the polarization of its nucleon parent in interactions at a given $Q^{2}$. The nucleon spin, $\left(\frac{1}{2}\right)$, can be decomposed as:

$$
\frac{1}{2}=\frac{1}{2}\left(\Delta \Sigma\left(Q^{2}\right)+\Delta g\left(Q^{2}\right)+L_{q}\left(Q^{2}\right)+L_{g}\left(Q^{2}\right)\right),
$$

where $\Delta \Sigma=\Delta u+\Delta d+\Delta s+\ldots$ and $\Delta g$ are the integrated net quark and gluon helicities along the nucleon spin direction, e.g.,

$$
\begin{aligned}
& \Delta u\left(Q^{2}\right) \equiv \int_{0}^{1} \delta u\left(x, Q^{2}\right) d x, \\
& \Delta g\left(Q^{2}\right) \equiv \int_{0}^{1} \delta g\left(x, Q^{2}\right) d x .
\end{aligned}
$$

The $L_{q}$ and $L_{g}$ represent the relative orbital angular momenta of the quarks and gluons, respectively.

To date, the only charged lepton polarized DIS data comes from non-collider experiments, running at energies where photon exchange dominates. Thus, only the parity conserving polarized structure functions $g_{1}^{\ell N}$ and $g_{2}^{\ell N}$ have 
been studied so far. The results are surprising[?]. From the measured first moment of $g_{1}^{\ell N}$ structure function, it can be concluded that only $30 \%$ of the nucleon spin is carried by quarks if one assumes $\Delta g=0$, i.e., $\Delta \Sigma=0.3$. An additional conclusion in this simple parton model interpretation is that the strange sea is anti-aligned with the nucleon spin, $\Delta s=-0.1$. In the more realistic QCD-enhanced parton model, the QCD evolution of the quark distributions brings in contributions from gluon radiation $(q \rightarrow q g)$ and pairproduction $(g \rightarrow q \bar{q})$ that induce a non-zero gluon spin distribution. This latter conclusion is supported by pQCD analyses of the $Q^{2}$ evolution of the available data on $g_{1}^{\ell N}$, as is illustrated in Fig. 6 .

Although the decomposition of Fig. 6 is highly model dependent, the ability of neutrinos to select specific quark flavors should allow a polarized DIS experiment at a muon storage ring to greatly constrain such models.

\subsection{Experimental Setup and Measurement Technique}

An ideal polarized target would have a mass of at least $10 \mathrm{~kg}$, high polarizability, a large fraction $f$ of polarizable material, and the capability for polarizing both protons and neutrons. The solid HD compound material "ICE" appears to be a promising new technology [41]. Both the $H$ and the $D$ can be polarized, either in separate experimental runs or together. The expected polarization and dilution are $P_{H}=80 \%$ and $f_{H}=1 / 3$ for hydrogen, and $P_{D}=50 \%$ and $f_{D}=2 / 3$ for deuterium. A $7 \mathrm{~kg}$ (density $\rho_{t}=0.11 \mathrm{~g} / \mathrm{cm}^{3}$ ) polarized target with the qualities mentioned above can be built out of an ICE-target that is $20 \mathrm{~cm}$ in radius and $50 \mathrm{~cm}$ deep, perhaps mounted upstream from a general purpose neutrino detector like that in Fig. 2. Raw event rates in such a polarized target could be of order $10^{8}$ events per year, as shown in Table 2. A previous study for $\nu$ MCs [42] has shown that a $200 \mathrm{~kg}$ target of polarized butanol with $10 \%$ polarization could measure the strange sea polarization to about $3 \%$ in a one year run in a beam downstream of a $250 \mathrm{GeV}$ muon collider with a $10 \mathrm{~m}$ straight section.

Measurement of $g_{1}$ requires both $\nu$ and $\bar{\nu}$ beams and follows from the double cross section difference:

$$
A_{g_{1}}=\left\{\sigma_{\uparrow \uparrow}^{\nu N}-\sigma_{\uparrow \downarrow}^{\nu N}\right\}-\left\{\sigma_{\uparrow \uparrow}^{\bar{\nu} N}-\sigma_{\uparrow \downarrow}^{\bar{\nu} N}\right\}
$$

where $\sigma_{\uparrow \uparrow}^{\nu N}$ denotes, for example, the neutrino scattering off a target $N$ with its spin polarized parallel to the neutrino helicity. This difference should be measured as a function of $x$ and $y$ to test for effects of unwanted PSF such as $g_{2}$. For $g_{3}$ one evaluates the similar quantity

$$
A_{g_{3}}=\left\{\sigma_{\uparrow \uparrow}^{\nu N}-\sigma_{\uparrow \downarrow}^{\nu N}\right\}+\left\{\sigma_{\uparrow \uparrow}^{\bar{\nu} N}-\sigma_{\uparrow \downarrow}^{\bar{\nu} N}\right\}
$$




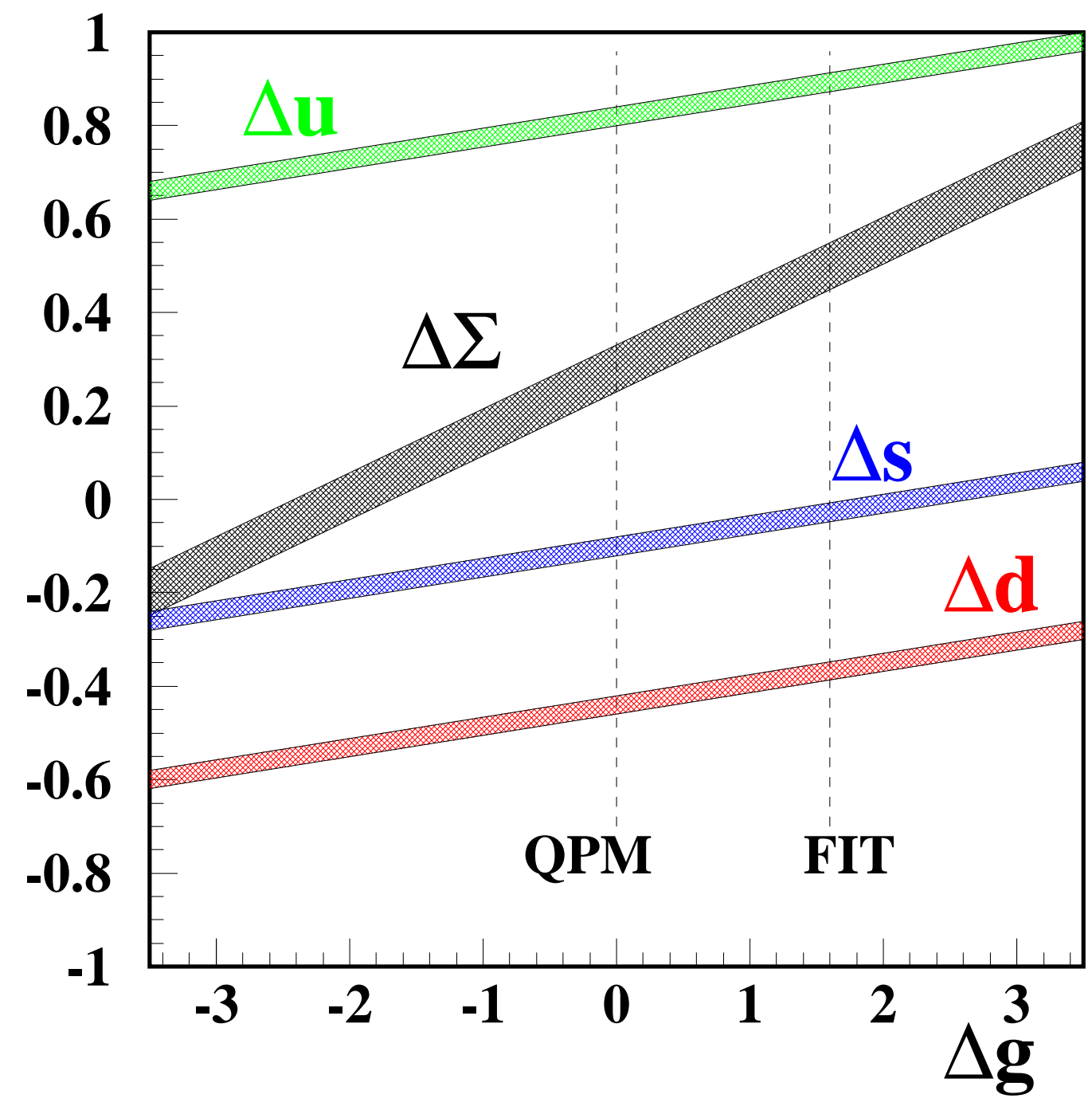

Fig. 6. Model-dependent decomposition of nucleon spin into contributions from quarks and gluons. The two vertical dotted lines show the naive QPM expectation and the results from a NLO fit to most of the available data on $g_{1}^{l}$.

Cross checks can be made with the target transversely polarized, where the suppression factors for the unwanted PSF are different.

\subsection{Applications of Polarized Parton Distribution Data from $\nu M C s$}

Measured values for $g_{1}$ from any nucleon target at a $\nu \mathrm{MC}$ would allow verification of the predicted decomposition:

$$
\int_{0}^{1} d x\left(g_{1}^{\nu N}+g_{1}^{\bar{\nu} N}\right)=\Delta \Sigma-C \Delta g
$$


where the factor $C$ is model dependent and a common choice is $C=N_{f} \alpha_{s} / 2 \pi$, and one has knowledge of $\Delta \Sigma$ from other data. In contrast to polarized charged lepton scattering, this determination can be done without relying on low energy input from beta decay data augmented by $\mathrm{SU}(3)$ symmetry.

For another application, one notes that $g_{3}, g_{4}$ and $g_{5}$ probe only non-singlet combinations of parton densities so that they do not get a contribution from the gluon density. Then, for scattering off an isoscalar target:

$$
x g_{3}^{\nu N}-x g_{3}^{\bar{\nu} N}=\delta c+\delta \bar{c}-\delta s-\delta \bar{s}
$$

(This is the polarized target analog of Eq. 20.) Assuming that $\delta c \ll \delta s$, this provides a measure of the level of polarization of the strange quark sea. Like $x F_{3}$, the non-singlet PSF, $g_{3}, g_{4}$ and $g_{5}$, have QCD evolutions containing no contribution from gluons at lowest order. Comparison of the non-singlet functions with the singlet SF $g_{1}$ and $F_{2}$ should therefore provide an indirect means for measuring the gluon contribution $\Delta g$.

As was the case for unpolarized targets, final state quark flavor tagging should provide additional semi-inclusive structure functions to augment the information on quark content from inclusive polarized structure functions. Probably the most important example will be studies of the strange spin contribution via charm production. Figures 4 and 5 show that approximately $5 \%$ of the events from a $50 \mathrm{GeV}$ muon storage ring will have charm in the final state, with $20 \%$ of these decaying semi-leptonically. An experiment with 20 million neutrino interactions thus will have $2 \times 10^{5}$ semi-leptonic charm events before kinematic cuts, which should be sufficient for a precise measurement of $\delta s$ and $\delta \bar{s}$.

\subsection{Studying Nuclear Effects with Neutrinos}

Nuclear effects in DIS have been studied extensively using muon and electron beams but have only been glanced at for neutrinos in low-statistics bubble chamber experiments. Neutrino experiments with high statisticss have formerly only been possible using heavy nuclear targets such as iron calorimeters and, for these targets, nuclear effects in $\nu N$ interactions have typically been considered as problems to overcome rather than as a source of physics insights. This section reviews the physics of nuclear QCD that is relevant to neutrino interactions and shows that $\nu$ MCs could instead provide experimental conditions where a great deal of interesting knowledge could be added to this field by using a variety of heavy nuclear targets as well as $H_{2}$ and $D_{2}$.

Nuclear studies at a $\nu \mathrm{MC}$ could use a general purpose detector such as that in Fig. 2 if it could be designed with interchangeable targets. Alternatively, 
a detector dedicated to nuclear studies could be used, perhaps with a geometry similar to that of the Fermilab E-665 Tevatron muon experiment[43]. For example, it could consist of a liquid $\mathrm{H}_{2}$ or $\mathrm{D}_{2}$ target followed by a rotating support of targets with different $A$ interspersed with tracking chambers, and then by an appropriate calorimeter/muon spectrometer. A $\nu \mathrm{MC}$ should be easily capable of supplying the event sample sizes required to examine the predicted nuclear effects. For example, one can consider a one-year exposure of each target to the beam in the $50 \mathrm{GeV} \nu \mathrm{MC}$ scenario of Table 1 . Then $10^{7}$ events would be acquired in targets subtending out to the $1 / \gamma_{\mu}$ characteristic angular size of the neutrino beam and that had lengths of $18 \mathrm{~cm}$ for $D_{2}, 1.4$ $\mathrm{cm}$ for graphite and only $0.16 \mathrm{~cm}$ for tungsten.

Different types of nuclear effects arise on passing through four distinct regions in Bjorken $x$ :

(1) "shadowing" for $x<0.1$,

(2) "anti-shadowing" for $0.1<x<0.2$,

(3) the "EMC Effect" for $0.2<x<0.7$, and

(4) "Fermi motion" for $x>0.7$.

These regions will now be discussed in turn.

\subsubsection{Low $x$ : PCAC and Nuclear Shadowing}

In the shadowing region, $x<0.1$, there are several effects where a neutrino probe could provide different insights to charged lepton probes. Considering first the limit as $Q^{2} \rightarrow 0$, the vector current is conserved and goes to zero but the axial-vector part of the weak current is only partially conserved (PCAC) and $F_{2}\left(x, Q^{2}\right)$ approaches a non-zero constant value as $Q^{2} \rightarrow 0$. According to the Adler theorem [31], $\sigma_{\nu N}$ can be related to to $\sigma_{\pi N}$ at $Q^{2}=0$. A $\nu \mathrm{MC}$ should be able to address the question of what effect a nuclear environment has on the Adler theorem.

The region of vector meson dominance (VMD) is reached in nuclear scattering of charged leptons ( $\ell^{ \pm} A$ scattering) in the low- $x$ shadowing regime as $Q^{2}$ increases from 0 but remains below of order $10 \mathrm{GeV}^{2}$. The physics concept of VMD is the dissociation of a virtual photon into a $q-\bar{q}$ pair, one of which interacts strongly with the "surface" nucleons of the target nucleus. Thus the interior nucleons are shadowed by the surface nucleons. Neutrino scattering should involve not only a VMD effect (though now with dissociation of a virtual $W$ rather than a photon) but also additional contributions from axial-vector mesons such as the $A_{1}$. Further, there should be additional non-perturbative effects that appear as nuclear shadowing (mainly in large nuclei) and which involve gluon recombination from nucleons neighboring the struck nucleon that shift the parton distributions towards higher values of $x$. 
Boros et al. [44] predict that the resulting shadowing effects in $\nu_{\ell} A$ scattering will be roughly $1 / 2$ that measured in $\ell^{ \pm} A$ scattering. A more quantitative analysis by Kulagin[45] uses a non-perturbative parton model to predict that shadowing in $\nu_{\ell} A$ scattering to be either equal to or slightly above that in $\ell^{ \pm} A$ scattering at $Q^{2}=5 \mathrm{GeV}^{2}$. It also attempts to determine the quark flavor dependence of shadowing effects by separately predicting shadowing for $F_{2 N C}^{\nu A}\left(x, Q^{2}\right), F_{2 N C}^{\nu A}\left(x, Q^{2}\right)$ and $x F_{3 C C}^{\nu A}\left(x, Q^{2}\right)$.

The predictions of Kulagin should be testable at a $\nu \mathrm{MC}$, as is indicated by Fig. 7. This shows a simulation for a $\nu \mathrm{MC}$ data sample of the predicted measured ratios of the values for $F_{2}(x)$ and $F_{3}(x)$ on a calcium target divided by those on a deuterium target:

$$
\begin{aligned}
& R_{2 A}(x) \equiv F_{2}\left(x, Q^{2}\right)[C a] / F_{2}\left(x, Q^{2}\right)\left[D_{2}\right], \\
& R_{3 A}(x) \equiv F_{3}\left(x, Q^{2}\right)[C a] / F_{3}\left(x, Q^{2}\right)\left[D_{2}\right],
\end{aligned}
$$

where the ratios are an event-weighted average over the experimental $Q^{2}$ range. As can be seen, the predicted difference between the shadowing on sea and valence quarks is clearly visible down to $x=0.02-0.03$.

\subsubsection{Mid $x$ : Anti-shadowing and the EMC Effect}

Drell-Yan experiments have also measured nuclear effects and their results are quite similar to DIS experiments in the shadowing region. However, in the anti-shadowing region where $R_{2 A}$ makes a brief but statistically significant excursion above 1.0 in DIS, Drell-Yan experiments see no effect. This could be an indication of difference in nuclear effects between valence and sea quarks.

Eskola et al. [46] have quantified this difference using a model which predicts that the differences between nuclear effects in $x F_{3 C C}^{\nu A}\left(x, Q^{2}\right)$ and $F_{2 C C}^{\nu A}\left(x, Q^{2}\right)$ should persist through the anti-shadowing region as well. Taking the work of Kulagin and Eskola together implies that nuclear effects in $x F_{3 C C}^{\nu A}\left(x, Q^{2}\right)$ should be quite dramatic, with more shadowing than $F_{2 C C}^{\nu A}\left(x, Q^{2}\right)$ at lower $x$ followed by $R_{3 A}$ rising fairly rapidly to yield significant anti-shadowing around $x=0.1$. The $50 \mathrm{GeV} \nu \mathrm{MC}$ experiment assumed for Figure 7 should be able to measure anti-shadowing effects and the difference between shadowing effects in $F_{2}\left(x, Q^{2}\right)$ and $x F_{3}\left(x, Q^{2}\right)$ at a statistical level of about 6 standard deviations.

\subsubsection{High x: Multi-quark Cluster Effects}

Analyses from DIS experiments of $F_{2}\left(x, Q^{2}\right)$ in the "Fermi-motion" region, $x>0.7$, have required few-nucleon correlation models and multi-quark cluster models to fit the data. These models boost the momentum of some of the quarks, producing a high- $x$ tail in $F_{2}\left(x, Q^{2}\right)$ that is predicted to behave as 


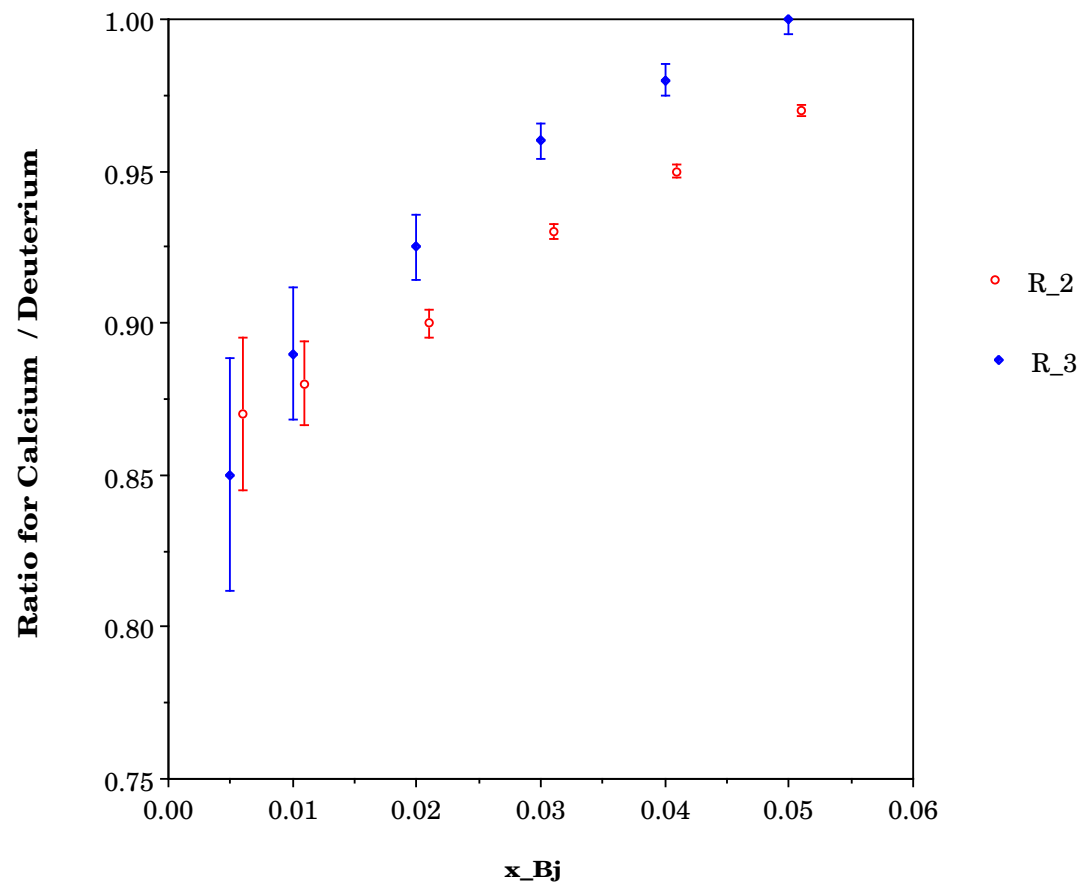

Fig. 7. Simulated predictions for the ratios of both the $F_{2}$ and $x F_{3}$ structure functions between calcium and deuterium, plotted as a function of Bjorken $x$ and assuming the theoretical model of Kulagin [45] and data samples from a $\nu \mathrm{MC}$. The sizes of the error bars correspond approximately to 1-year exposures for both targets at the $50 \mathrm{GeV} \nu \mathrm{MC}$ of Table 1 .

$e^{-a x}$. However, fits to $\mu C a$ [47] and $\nu F e$ [48] have obtained two different values for the fitted constant $a: a_{\mu C a}=16.5 \pm 0.5$ and $a_{\nu F e}=8.3 \pm 0.7 \pm$ 0.7 , respectively. This was considered surprising because of the expectation that any few-nucleon-correlation or multi-quark effects would have already saturated by carbon. A high statistics data sample from a $\nu \mathrm{MC}$, with precise kinematic reconstruction of the Bjorken $x$ values for each event, could go a long way towards resolving the dependence of the value of $a$ on the nucleus and on the lepton probe.

\section{Studies of the CKM Quark Mixing Matrix}




\subsection{Introduction}

The CKM quark mixing matrix comprises a set of fundamental parameters of the Standard Model that reflect the dynamics shaping the generation of particle masses. Furthermore, it provides essential input for predictions on various $\mathrm{CP}$ asymmetries in $B$ decays which should be sharpened as much as possible in order to increase their sensitivity to the presence of new physics processes.

It is likely that $\nu \mathrm{MCs}$ could make a very substantial contribution to measurements of quark mixing, with systematically unique measurements of four of the nine moduli in the CKM matrix that should be comparable to or better than the best future measurements at other experiments. The relevant moduli are $\left|V_{c d}\right|,\left|V_{c s}\right|,\left|V_{u b}\right|$, and $\left|V_{c b}\right|$, to be extracted from charm production off $d$ and $s$ quarks, and from beauty production off $u$ and $c$ quarks, respectively. If muon colliders ever reach the $100 \mathrm{TeV}$ energy center-of-mass range then their $\nu \mathrm{MCs}$ could further provide a unique opportunity for precise direct measurements of the CKM elements involving the top quark [14].

This section is organized as follows. Section 3.1.1 gives a summary of the current experimental status and future expectations for before the advent of $\nu \mathrm{MCs}$. Section 3.1.2 then gives a general overview of the method for extracting CKM information at $\nu$ MCs . Sections 3.2 and 3.3 then give more specific details on $\nu \mathrm{MC}$ experimental analyses involving the production of charm and bottom quarks, respectively. A further analysis that may have potential for a measurement of CKM parameters - diffractive charmed vector meson production - is briefly addressed in subsection 3.4. Finally, subsection 3.5 summarizes all of these expected improvements from $\nu$ MCs to our knowledge of the CKM matrix.

\subsubsection{Current Experimental Knowledge of the Relevant CKM Matrix Ele- ments, and Future Expectations}

Table 3 presented the current [15] experimental values and uncertainties for the absolute squares of the interesting CKM matrix elements for $\nu \mathrm{MCs}: V_{c d}$, $V_{c s}, V_{u b}$ and $V_{c b}$. The first two of these can be more tightly constrained by adding the assumption of three family unitarity, so improved measurements of these elements can be considered as testing these unitarity conditions.

The values for $\left|V_{c d}\right|$ and $\left|V_{c s}\right|$ have been extracted from charm production in deep inelastic scattering supplemented by information gathered in semileptonic $D$ decays, while $\left|V_{c b}\right|$ and $\left|V_{u b}\right|$ have been obtained from semileptonic $B$ decays. 
In the next few years, exclusive semileptonic charm decays like $D \rightarrow \ell \nu K /$ $K^{*} / \pi / \rho$ will be measured more precisely. However it is not clear at present whether theoretical technologies like QCD simulations on the lattice will improve sufficiently to fully utilize such data for extracting more accurate values of $\left|V_{c s}\right|$ and $\left|V_{c d}\right|$.

At present $\left|V_{c b}\right|$ is measured with about a $5 \%$ theoretical uncertainty and a $5 \%$ or better experimental uncertainty. (For comparisons with the uncertainties quoted in Table 3 recall that the fractional uncertainty in the square of the modulus is twice that of the modulus itself.) The two most reliable determinations are from the semileptonic $B$ width and the form factor for $B \rightarrow \ell \nu D^{*}$ at zero recoil. One can reasonably expect the theoretical uncertainty in these processes to go down to about $2 \%$ [50].

The situation is considerably less satisfactory for $\left|V_{u b}\right|$. Most of the analyses employed so far contain a large dependence on theoretical models, the accuracy of which is hard to evaluate. One can hope that studies of both exclusive and inclusive semileptonic $B$ decays done at the $B$ factories will yield values for $\left|V_{u b}\right|$ with a theoretical accuracy of no more than $10 \%$ by 2005 . However, this is not guaranteed.

It is highly desirable to improve on this situation by obtaining more precise and reliable values for these CKM parameters. The main reason is that they are theoretically expected to be a consequence of the generation of quark masses which in turn reflects dynamics operating at presumably ultra-high energy scales. It turns out that qualitatively quite distinct "textures" for the Yukawa couplings assumed to apply at GUT scales lead to CKM parameters that, due to differing renormalization effects, are numerically relatively similar at electroweak scales. Secondly, various CP asymmetries in $B$ decays will be measured presumably to better than $5 \%$ over the next ten years; and their predicted values depend crucially on the size of $V_{u b}, V_{c b}$ (and $V_{t d}$ ). To exploit the discovery potential for new physics to the fullest one wants to match up experimental and theoretical accuracy.

To be more specific:

- One wants at least to confirm the values for $\left|V_{c s}\right|$ and $\left|V_{c b}\right|$ by a systematically different method.

- One would like to determine a precise value for $\left|V_{c d}\right|$ without imposing threefamily-unitarity.

- It is an important goal to extract the value of $\left|V_{u b}\right|$ with considerably less than $10 \%$ uncertainty.

It appears that $\nu \mathrm{MCs}$ are up to these tasks and possibly more. Indeed, if a future $\nu \mathrm{MC}$ in the $100 \mathrm{TeV}$ energy range were ever to be realized, then even top quark production could be studied and this would uniquely allow the 
extraction [14] of $V_{t d}$ and $V_{t s}$ in a relatively straightforward way!

\subsubsection{Extracting CKM Matrix Elements in $\nu M C s$ : an Overview}

The experimental quantities used for all the quark mixing measurements in $\nu \mathrm{MCs}$ are the production cross sections and kinematic distributions of heavy flavor final states from CC interactions. These are related back to the quark couplings through a parameterization of the initial state quark distributions and a model of the production processes that includes threshold suppressions due to the quark masses. Measurements of CKM matrix elements at $\nu \mathrm{MCs}$ will be analogous to, but vastly superior to, current neutrino measurements of $\left|V_{c d}\right|^{2}$ that use dimuon events for final state tagging of charm quarks, which are reviewed elsewhere[12]. This improvement is due to the overall higher rate of interactions, the ability to tag charm inclusively by vertexing, and the capability of fully reconstructing the event kinematics for charm decays to hadrons.

In principle, the struck quark can be converted into any of the three final state quarks that differ by one unit of charge. In practice, production of the heavy top quark is kinematically forbidden except from neutrinos at energies above about $16 \mathrm{TeV}$, and the production of other quark flavors is influenced by their mass. After correcting for this kinematic suppression, the Standard Model predicts the probability for the interaction to be proportional to the absolute square of the appropriate element in the CKM matrix. The most relevant theoretical uncertainty lies in the treatment of the production thresholds. Measuring these cross sections at different energies and separately for CC and $\mathrm{NC}$ reactions should help in understanding the threshold behavior.

\subsection{Analyses Involving Charm Production: the Extraction of $V_{c d}$ and $V_{c s}$}

The extraction of $\left|V_{c d}\right|$ will be the cleanest of the four CKM measurements at $\nu$ MCs discussed here and is the one CKM element whose modulus is already best determined in neutrino-nucleon scattering. It is measured from charm production off valence $d$ quarks in isoscalar targets, and this valence quark distribution can be accurately determined from nucleon structure function measurements.

Figures 4 and 5 of Sec. 1 show that charm production occurs in $\sim 7 \%$ of the CC interactions from $100 \mathrm{GeV}$ neutrinos, and will occur at slightly lower/higher rates for lower/higher energies according to the changing level of mass threshold suppression. This implies that realistic charmed event sample sizes might reach the $10^{8}$ level. 
The feature of the $\left|V_{c d}\right|$ analysis that makes it systematically rather clean is that charm production from valence quarks occurs for neutrinos but not for antineutrinos. Valence quarks have a harder $x$ distribution than sea contributions from both $s$ and (at a lower level) $d$ quarks. It is also helpful that the $s$ and $d$ seas seen by neutrinos are also closely equal to their antiquark counterparts that are probed by antineutrinos, as would be verified in structure function analyses of the $\nu \mathrm{MC}$ data set ( Sec. 2). This allows use of the $\bar{\nu}$ sample as an effective background subtraction for the sea contributions to the charm production by neutrinos.

To illustrate, one can measure scattering from an isoscalar target $D$ with $\nu / \bar{\nu}$ beams and extract the ratio

$$
r_{c}(x, y, E)=\frac{d \sigma_{\mu c X}^{\nu D} / d x d y-d \sigma_{\mu c X}^{\bar{\nu} D} / d x d y}{d \sigma_{\mu X}^{\nu D} / d x d y-d \sigma_{\mu X}^{\bar{\nu} D} / d x d y},
$$

where $d \sigma_{\mu c X}^{\nu D(\bar{\nu} D)} / d x d y$ represent the charm production cross sections, and $d \sigma_{\mu c X}^{\nu D(\bar{\nu} D)} / d x d y$ denote the total inclusive CC cross sections. To leading order in QCD, this ratio can be computed from

$$
\frac{1}{r_{c}^{L O}(x, y, E)}=\frac{\left(q\left(x, Q^{2}\right)-\bar{q}\left(x, Q^{2}\right)\right)\left(\left|V_{u d}\right|^{2}-(1-y)^{2}\right)}{\left|V_{c d}\right|^{2}\left(q\left(\xi, Q^{2}\right)-\bar{q}\left(\xi, Q^{2}\right)\right)(1-y+x y / \xi)}+1
$$

where $q\left(x, Q^{2}\right)=\left(u\left(x, Q^{2}\right)+d\left(x, Q^{2}\right)\right) / 2, \xi=x\left(1+m_{c}^{2} / Q^{2}\right)$, and $m_{c}$ is the charm quark mass. Note that $r_{c}^{L O}(x, y, E)$ depends only on the precisely measured $\left|V_{u d}\right|^{2}$, the well understood valence quark distribution, $q\left(\xi, Q^{2}\right)-$ $\bar{q}\left(\xi, Q^{2}\right)$, the charm mass, and $\left|V_{c d}\right|^{2}$. Because the valence distribution peaks at relatively high $x$, threshold effects associated with $m_{c}$ are minimized. Nextto-leading-order calculations are more complicated but leave these features intact.

As in analyses of today's neutrino experiments, the $d$ and $s$ contributions to the charm event sample will in practice be separated from one another in a fit involving the measured $x$ distributions of the events and with the charm quark mass as a fitted parameter. With such huge statistics, the statistical uncertainty in the measurement will be only at the $10^{-4}$ level so the measurement will be dominated by systematic uncertainties. Major contributions to the uncertainty are likely to be:

- Estimation of the charm tagging efficiency. This error is minimized by the clean, high efficiency charm tagging that is possible with high performance vertexing and a negligible $B$ hadron background[9].

- The charm production model. It is a very attractive feature that the measurement is effectively of the ratio of charm production to the total production in each kinematical bin. Therefore, one is sensitive only to the kinematical suppression of charm states and not to the initial parton distribution 
functions. Mass threshold corrections that are applied according to theoretical models can be checked and calibrated from the trends in the data itself over the range of kinematical bins. Therefore, this uncertainty should initially decrease with increasing sample size.

- Estimation of the extent to which the sea is symmetric in quarks versus antiquarks. This uncertainty is lessened because the signal is largely at higher $x$ than the background. If helpful, a cut in $x$ value could be applied to the fit to further reduce this uncertainty.

With experimental handles on the major systematic uncertainties, it may be guessed that the irreducible theoretical uncertainty on $\left|V_{c d}\right|^{2}$ due to violations of quark-hadron duality might be at the percent level.

A measurement of $\left|V_{c s}\right|^{2}$ presents more challenges because this matrix element always appears in combination with the strange quark PDF $s\left(x, Q^{2}\right)$, which is difficult, but not impossible, to measure separately. Perhaps the best scheme ${ }^{3}$ involves a simultaneous analysis of inclusive charged current scattering and semi-inclusive charm production[51] on deuterium targets. The inclusive CC cross section is of the form

$$
\frac{d \sigma_{\mu X}^{\nu D(\bar{\nu} D)}}{d x d y}=\frac{d \sigma_{\mu c X}^{\nu D(\bar{\nu} D)}}{d x d y}+\frac{d \sigma_{\mu \phi X}^{\nu D(\bar{\nu} D)}}{d x d y}
$$

where $d \sigma_{\mu q X} / d x d y$ represents the part of the cross section with no charm in the final state ( $b$ production is neglected). To leading order

$$
\begin{aligned}
\frac{\pi}{2 G_{F}^{2} M E} \frac{d \sigma_{\mu c X}^{\nu D}}{d x d y} & =\left[\left|V_{c s}\right|^{2} s\left(\xi, Q^{2}\right)+\left|V_{c d}\right|^{2} q\left(\xi, Q^{2}\right)\right]\left(1-\frac{m_{c}^{2}}{2 M E \xi}\right) \\
\frac{\pi}{2 G_{F}^{2} M E} \frac{d \sigma_{\mu c X}^{\bar{\nu} D}}{d x d y} & =\left[\left|V_{c s}\right|^{2} \bar{s}\left(\xi, Q^{2}\right)+\left|V_{c d}\right|^{2} \bar{q}\left(\xi, Q^{2}\right)\right]\left(1-\frac{m_{c}^{2}}{2 M E \xi}\right) \\
\frac{\pi}{2 G_{F}^{2} M E} \frac{d \sigma_{\mu q X}^{\nu D}}{d x d y} & =\left|V_{u d}\right|^{2} q\left(x, Q^{2}\right)+\left|V_{u s}\right|^{2} s\left(x, Q^{2}\right) \\
& +\bar{q}\left(x, Q^{2}\right)(1-y)^{2} \\
\frac{\pi}{2 G_{F}^{2} M E} \frac{d \sigma_{\mu q X}^{\bar{\nu} D}}{d x d y} & =\left|V_{u d}\right|^{2} \bar{q}\left(x, Q^{2}\right)+\left|V_{u s}\right|^{2} \bar{s}\left(x, Q^{2}\right) \\
& +q\left(x, Q^{2}\right)(1-y)^{2}
\end{aligned}
$$

Measuring $d \sigma_{\mu X}^{\nu(\bar{\nu})} / d x d y$ and $d \sigma_{\mu c X}^{\nu(\bar{\nu})} / d x d y$ gives $d \sigma_{\mu q X}^{\nu(\bar{\nu})} / d x d y$. The $y$ dependencies of the non-charm cross sections allow measurement of $q\left(x, Q^{2}\right)$ and $\bar{q}\left(x, Q^{2}\right)$. Assuming that $\left|V_{u d}\right|^{2}$ and $\left|V_{u s}\right|^{2}$ contribute negligible error, and that $s\left(x, Q^{2}\right)=$ $\bar{s}\left(x, Q^{2}\right)$, one then extracts $s\left(x, Q^{2}\right)$ from the Cabibbo suppressed $s \rightarrow u$ pieces of $d \sigma_{\mu q X}^{\nu(\bar{\nu})} / d x d y$, and then $\left|V_{c s}\right|^{2}$ (and $\left|V_{c d}\right|^{2}$ ) from $d \sigma_{\mu c X}^{\nu(\bar{\nu})} / d x d y$.

$\overline{3}$ Methods to extract $s\left(x, Q^{2}\right)$ discussed in Sec. 2 assume a known CKM matrix. 
This procedure will require very high statistics and extreme care with theoretical and experimental systematic errors as it turns on the $\left|V_{u s}\right|^{2} s\left(x, Q^{2}\right)$ term in the cross section, which contributes only $\sim 0.15 \%$ of the total cross section. Inclusive and charm cross sections must be cross-normalized to high accuracy, and the expressions (45-48) must be generalized to include higher order QCD and possible non-perturbative effects.

Additionally, it may also be possible to measure the total strange quark content of the nucleon in NC "leading particle tagging" with a $\phi(1020)$ final state [9], although no experience with this method yet exists in neutrino physics.

In the end, it will likely be difficult to compete with the indirect constraint on $\left|V_{c s}\right|^{2}$ from precise measurements of the hadronic width of the $W, \Gamma_{W}^{h}$, at LEP2, the Tevatron and the LHC. This observable is, to leading order,

$$
\Gamma_{W}^{h}=\frac{1}{3} \Gamma_{W}^{t o t} \sum_{Q=u, c} \sum_{q=d, s, b}\left|V_{Q q}\right|^{2}
$$

where $\Gamma_{W}^{t o t}$ is the total hadronic width. Because $\left|V_{u b}\right|^{2}$ is small and all other terms in the sum can be measured precisely elsewhere, a strong $\left|V_{c s}\right|^{2}$ constraint emerges.

\subsection{Analyses Involving Bottom Production: the Extraction of $V_{u b}$ and $V_{c b}$}

Measurements of $V_{u b}$ and $V_{c b}$ from the two transitions with a $b$ quark in the final state $-u \rightarrow b$ and $c \rightarrow b$, respectively - require analyses that are conceptually similar to those for charm discussed in the preceding subsection. Figures 4 and 5 show that statistics for CC $b$ quark production to be of order $10^{4}$ events for a total sample of $10^{10}$ inclusive events, depending on the energy of the muon storage ring and the consequent threshold suppression due to the $b$ quark mass. Besides the threshold suppression, the main reasons why the $B$ production levels are so low for the two processes are that, in the one case, the production from $u$ quarks is suppressed by $\left|V_{u b}\right|^{2} \sim \mathcal{O}\left(10^{-5}\right)$, while production from charm quarks is inhibited by $\left|V_{c b}\right|^{2} \simeq 1.6 \times 10^{-3}$ and the extra mass of the $\bar{b} c$ final state.

Given the nearly optimal vertexing geometry possible at a $\nu \mathrm{MC}$, separating out much of the fractionally small $b$ hadron event sample[9] from the charm background should be feasible. Assuming that the $B$ tagging efficiency and purity are well known, then the $b$ production analysis should be a relatively straightforward copy of that for charm, involving a simultaneous fit to the $u$ and $c$ contributions to $B$ production from neutrino and antineutrino scattering. 
In analogy to charm production, the resolving power of the fit arises because antineutrino (but not neutrino) interactions give a high $x$ contribution from $u$ valence quarks, while $c \rightarrow b$ transitions will be equal for $\nu$ and $\bar{\nu}$ and typically at lower $x$. It is again easy to see the effect at LO in QCD, as follows.

Define, in analogy to Eq. 42,

$$
r_{b}(x, y, E)=\frac{d \sigma_{\mu b X}^{\nu D} / d x d y-d \sigma_{\mu b X}^{\bar{\nu} D} / d x d y}{d \sigma_{\mu X}^{\nu D} / d x d y-d \sigma_{\mu X}^{\bar{\nu} D} / d x d y}
$$

where now $d \sigma_{\mu b X}^{\nu D(\bar{\nu} D)} / d x d y$ represents the beauty production cross sections. QCD then predicts

$$
\frac{1}{r_{b}^{L O}(x, y, E)}=-\frac{\left(q\left(x, Q^{2}\right)-\bar{q}\left(x, Q^{2}\right)\right)\left(\left|V_{u d}\right|^{2}-(1-y)^{2}\right)}{\left|V_{u b}\right|^{2}\left(q\left(\xi^{\prime}, Q^{2}\right)-\bar{q}\left(\xi^{\prime}, Q^{2}\right)\right)(1-y)\left(1-x y / \xi^{\prime}\right)}+1
$$

with $\xi^{\prime}=x\left(1+m_{b}^{2} / Q^{2}\right)$ and $m_{b}$ the $b$ quark mass. As was the case with charm, $r_{b}^{L O}(x, y, E)$ depends only on the high $x$ valence quarks, which helps reduce the more substantial suppression associated with the higher value of $m_{b}$.

Reasonably accurate estimates of the measurement precisions would require both a more detailed study and a knowledge of the expected event sample size. The precision of the $\left|V_{u b}\right|^{2}$ measurement may approach the statistical limit of around $1 \%$ for a total neutrino event sample of order $10^{10}$ events.

The $c \rightarrow b$ transition can (unlike $s \rightarrow c$ ) be identified experimentally by the presence of soft $c$ quark observed in association with the $\bar{b}$ quark in $\nu$ scattering. At $\nu \mathrm{MC}$ energies, it is reasonable to assume that $\bar{b} c$ production will be dominated by $W$-gluon fusion, and a good knowledge of the gluon PDF at high $x$ will be required. This may well limit the $\left|V_{c b}\right|^{2}$ measurement accuracy to the few percent level.

It is widely expected that $\left|V_{c b}\right|$ will be known from $B$ decays in other experiments to a few percent or better by 2005 [50]. Given this, an alternative and maybe more useful analysis strategy for a $\nu \mathrm{MC}$ might be to combine the $V_{c b}$ analysis data at the $\nu \mathrm{MC}$ with the value of $V_{c b}$ as extracted from semileptonic $B$ decays in other experiments, with the purpose of analyzing the mass suppression in beauty production close to threshold, or in constraining the gluon PDF. This information could then be used as input to the extraction of $V_{u b}$ as sketched above. 


\section{$3.4 V_{c d} / V_{c s}$ via Diffractive Charmed Vector Meson Production}

It is possible to diffractively produce charmed vector mesons via $W$-bosonpomeron scattering[52,53]:

$$
\begin{aligned}
& \nu_{\mu} A \rightarrow \mu^{-}\left(W^{+} \mathcal{P}\right) A \rightarrow \mu^{-} D_{S}^{*+} A, \\
& \nu_{\mu} A \rightarrow \mu^{-}\left(W^{+} \mathcal{P}\right) A \rightarrow \mu^{-} D^{*+} A .
\end{aligned}
$$

The $D_{S}^{*+}$ cross section is of order $0.01 \mathrm{fb} /$ nucleus, while that for $D^{*+}$ should be smaller by a factor of $\left|V_{c d} / V_{c s}\right|^{2}$. For $10^{10} \mathrm{CC}$ events, one expects of order $1.5 \times 10^{6} D_{S}^{*}$ events and $7500 D^{*}$ events. If the $W-D_{S}^{*}$ and $W-D^{*}$ dynamical couplings are identical, the relative rate can measure the ratio of the CKM matrix elements.

The experimental signature is fairly unique: a two- or three-prong muon-meson vertex with a $D^{+} / D^{0}$ or $D_{S}^{+}$secondary. The cross section will peak at low momentum transfer and there should be no evidence for nuclear breakup.

The chief theoretical uncertainty likely has to do with evaluating SU(3)breaking effects in the $W$-vector meson couplings and this may limit the potential for CKM studies from this process. Again, an alternative perspective can be taken: to use the information available on $\left|V_{c d} / V_{c s}\right|^{2}$ to analyze the $S U(3)$ pattern of diffractive $D^{*}$ vs. $D_{s}^{*}$ production.

\subsection{Improved Knowledge of the CKM Matrix from $\nu M C s$}

The Particle Data Group[15] assigns the following uncertainties to the four CKM parameters that were the main focus of our discussion:

$$
\begin{aligned}
& \left|\Delta V_{c d}\right|_{\text {now }}=7 \% \quad[3 \%] \\
& \left|\Delta V_{c s}\right|_{\text {now }}=15 \% \quad[2 \%] \\
& \left|\Delta V_{\text {ub }}\right|_{\text {now }}=30 \% \\
& \left|\Delta V_{c b}\right|_{\text {now }}=8 \%
\end{aligned}
$$

where the numbers for $V_{c d}$ and $V_{c s}$ quoted in square brackets hold after 3-family unitarity has been imposed.

The future before a $\nu \mathrm{MC}$ could contribute can be sketched as follows:

- Some improvements can be expected over the next several years in direct extractions of $V_{c d}$ and $V_{c s}$ (i.e. those that do not impose 3-family unitarity constraints). However it is very unlikely that they could come close to the 2 - $3 \%$ level. 
- A combination of more detailed data on semileptonic $B$ decays and further refinement of heavy quark expansions will yield very significant improvements in $V_{c b}$ and $V_{u b}$; not unreasonable expectations are

$$
\left|\Delta V_{c b}\right|_{p r e-\nu M C} \simeq 3 \%,\left|\Delta V_{u b}\right|_{p r e-\nu M C} \simeq 10-15 \%
$$

Our discussion suggests that potential $\nu \mathrm{MC}$ analyses could have an essential impact on central aspects of the Standard Model by meeting the goals stated in the beginning of this section:

- provide a systematically different determination of $\left|V_{c s}\right|$ and $\left|V_{c b}\right|$ that is as good as can be achieved in charm and beauty decay studies:

$$
\left|\Delta V_{c s}\right|_{\nu M C} \sim \mathcal{O}(\text { few } \%),\left|\Delta V_{c b}\right|_{\nu M C} \sim \mathcal{O}(\text { few } \%) ;
$$

- yield a value for $\left|V_{c d}\right|$ through direct observation that is about as good as otherwise achieved only through imposing 3-family-unitarity

$$
\left|\Delta V_{c d}\right|_{\nu M C} \sim \mathcal{O}(1 \%)
$$

- lower the theoretical uncertainty in $\left|V_{u b}\right|$ considerably:

$$
\left|\Delta V_{u b}\right|_{\nu M C} \sim \mathcal{O}(1 \%)
$$

This would enable us to predict various $C P$ asymmetries in $B$ decays with order $1 \%$ accuracy, thus calibrating the experimental results expected from next generation experiments like LHC-B and BTeV and allowing us to exhaust the discovery potential for new physics in $B$ decays;

- improve dramatically our numerical information on the CKM parameters involving top quarks if a very high energy $\nu \mathrm{MC}$ could be built.

\section{Precision Electroweak Studies}

\subsection{Introduction}

Neutrino scattering is a natural place to study the structure of the weak interaction. Historically, it has played an important role in establishing both the basic structure of the weak interaction, particularly with the discovery of neutral currents [54], and in providing the first precision tests of electroweak unification [55]. It is reasonable, as the high intensity neutrino beams at future $\nu \mathrm{MCs}$ offer the promise of a new level of statistics in high energy neutrino interactions, that one considers a new generation of experiments to probe the weak interaction. 
At the same time, given the ambitious collider physics programs of LEP I, SLD, LEP II and the Tevatron, which will have been completed at the time of a $\nu \mathrm{MC}$, and given the physics program of the LHC that will be ongoing, the goals of such experiments must be correctly focused.

\subsubsection{Knowledge at the Time of $\nu M C s$}

Between today and the advent of a $\nu \mathrm{MC}$, it is safe to assume that little improvement will have been achieved in the amazingly precise measurements of $Z^{0}$ decay and production parameters [56]. However, it is very likely that LEP II and the Tevatron Run II will have produced a measurement of $M_{W}$ to a $20 \mathrm{MeV} / c^{2}$ precision[58,59] and that the mass of the top quark will be known with a precision of $1 \mathrm{GeV} / c^{2}[59]$.

Furthermore, LEP-II has found experimental hints of a possible Higgs boson at a mass around $115 \mathrm{GeV} / c^{2}$ which, although far from being a sure bet, might possibly be confirmed at either the Tevatron or the LHC. Alternatively, one of these hadron colliders might instead discover the Higgs at a higher mass or else something completely different. Of course, the odds of such a discovery are much higher if a Higgs exists close to the value hinted at by the LEP-II events, as present electroweak fits also seem to suggest [56].

An observation of a Standard Model Higgs, along with a precise prediction of its mass driven by the $W$ and top quark mass measurements, will make an elegant pièce de resistance of the electroweak physics program at the energy frontier for the period between 1990 and 2010.

During this time, there may also be improvements in low energy tests of the electroweak Standard Model. Atomic parity violation experiments may be able to yet again improve significantly in their ability to provide precision tests of weak interactions if experiments utilizing trapped unstable Francium become possible[60]. Also, SLAC E-158[61], a proposed polarized Moller scattering experiment, may be able to probe $\sin ^{2} \theta_{W}$ at a precision of 0.0008 at $Q^{2} \sim$ $10^{-2} \mathrm{GeV}^{2}$.

\subsubsection{Goals of Fixed-Target Electroweak Physics Programs}

The primary goals of a low energy test of a high energy theory remain largely the same as they have been in the past. Unification of the interactions of the on-shell weak bosons with their low energy manifestations in weak interactions at high precision remains an appealing and elegant test of the model.

The low energy experiments also allow access to some aspects of the theory that cannot be readily observed at high energies. For example, a demonstration 
of the running of the weak coupling strength now only awaits precise low energy data [62], given the high precision at the scale of the weak boson masses. Another powerful use for low energy data is the sensitivity to interference between new physics and tree-level processes. For example, if $Z^{\prime}$ bosons are discovered at the energy frontier then observation of the interference between the Standard Model $Z$ and the $Z^{\prime}$ at low energy may be one of the most powerful tests constraining models that relate the two interactions.

\subsubsection{Electroweak Processes with Neutrinos}

To provide a precision test of the Standard Model, a process must be reasonably common and precisely calculable. The two useful neutrino interaction processes for these sorts of studies are measurements of $\sin ^{2} \theta_{W}$ through neutrino-nucleon DIS (neutrino-quark scattering), and neutrino-electron elastic and quasi-elastic scattering. The former, of course, wins on large cross section, but the latter wins on simplicity of target and therefore minimal theoretical uncertainties. The possibilities for using these processes are described below.

Electroweak processes not considered here include neutrino tridents - i.e. three lepton final states resulting from internal conversion of a virtual photon which are effectively a test of boson-boson scattering. While these processes are interesting, they do not provide a stable basis on which to form a precision probe of the model because of large theoretical uncertainties in the cross section. See Sec. 5 for further discussion of this and other processes.

\subsection{Elastic and Quasi-Elastic Neutrino-Electron scattering}

Perhaps the most promising reaction for measuring $\sin ^{2} \theta_{W}$ at a $\nu \mathrm{MC}$ is $\nu-e$ scattering. Neutrino-electron scattering possesses one significant advantage over DIS for precision electroweak studies, namely that the target is point-like and its structure does not introduce uncertainties in extracting the parameters of the fundamental interaction from the observed cross sections. 


\subsubsection{Survey of Neutrino-Electron Scattering Processes}

Several $\nu-e$ scattering reactions will occur in the muon and electron neutrino and antineutrino beams at a $\nu \mathrm{MC}$ :

$$
\begin{aligned}
& \nu_{\mu} e^{-} \rightarrow \nu_{\mu} e^{-} \\
& \nu_{\mu} e^{-} \rightarrow \nu_{e} \mu^{-}, \\
& \bar{\nu}_{\mu} e^{-} \rightarrow \bar{\nu}_{\mu} e^{-} \\
& \nu_{e} e^{-} \rightarrow \nu_{e} e^{-} \\
& \bar{\nu}_{e} e^{-} \rightarrow \bar{\nu}_{e} e^{-}, \\
& \bar{\nu}_{e} e^{-} \rightarrow \bar{\nu}_{\mu} \mu^{-}, \bar{\nu}_{\tau} \tau^{-}, \bar{u} d \ldots
\end{aligned}
$$

Reactions 62 and 64 are purely neutral-current processes mediated by the exchange of a $Z^{0}$. Reaction 65 has both charged current ( $W^{ \pm}$exchange) and neutral current components, and reaction 66 has neutral current $t$-channel and charged-current $s$-channel components. Reactions 63 and 67 can result in the production of a single muon and therefore have a significant muon mass threshold in the cross section. Figure 8 shows the Feynman diagrams for these processes.

Because of the small ratio of the electron to proton mass, the cross section for neutrino-electron scattering is much smaller than that for neutrino-nucleon DIS. The leading order differential cross section for neutrino-electron elastic scattering with respect to $y=E_{e} / E_{\nu}$ is given by

$$
\frac{d \sigma}{d y}\left(\nu e^{-} \rightarrow \nu e^{-}\right)=\frac{G_{F}^{2} s}{\pi}\left[g_{L}^{2}+g_{R}^{2}(1-y)^{2}\right]
$$

where the center-of-momentum energy, $s$, is well appoximated by $s \simeq 2 m_{e} E_{\nu}$ when $E_{\nu} \gg m_{e}$, where terms of $\mathcal{O}\left(m_{e} / E_{\nu}\right)$ are neglected and where $g_{L}$ and $g_{R}$ are process dependent because of their exchange in the neutral current process under $\nu \leftrightarrow \bar{\nu}$ and because of the addition of the charged current process for electron neutrino induced reactions. The values of $g_{L}$ and $g_{R}$ are given in Table 4. Numerical values for the cross sections after integrating over $y$ are:

$$
\sigma\left(\nu e^{-} \rightarrow \nu e^{-}\right)=1.6 \times 10^{-41} \times E_{\nu}[G e V] \times\left[g_{L}^{2}+\frac{1}{3} g_{R}^{2}\right]
$$

where the values for the final term are given in the final column of Table 4. Radiative corrections for this process have been calculated to 1-loop [63], and theoretical techniques exist to extend this calculation to higher orders.

The differential cross section for inverse muon decay, Eq. 63, is

$$
\frac{d \sigma}{d y}\left(\nu_{\mu} e^{-} \rightarrow \nu_{e} \mu^{-}\right)=\frac{G_{F}^{2}\left(s-m_{\mu}^{2}\right)}{4 \pi},
$$



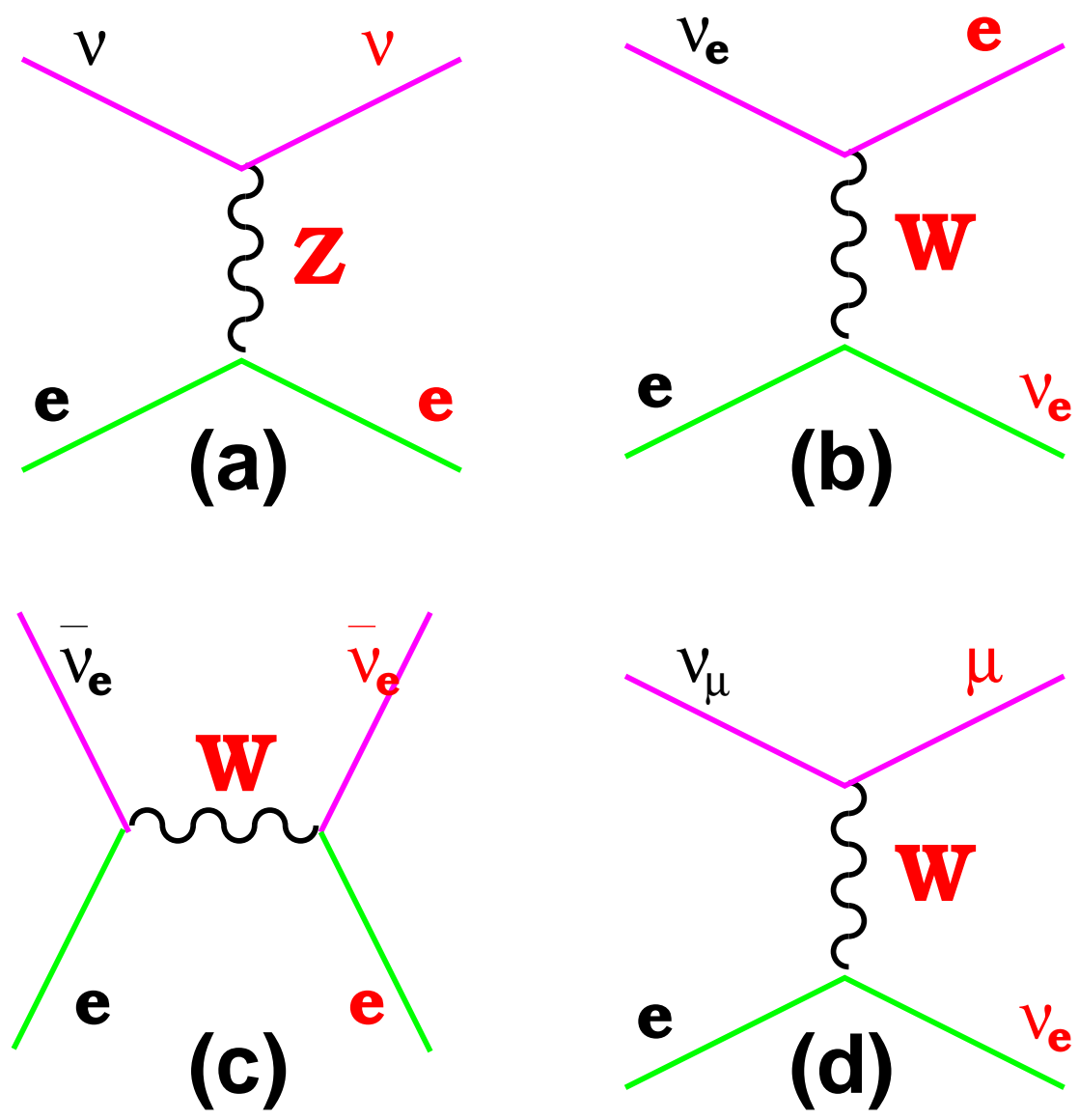

Fig. 8. Feynman diagrams contributing to the $\nu-e$ scattering processes of Eqs. 62 to 66: (a) NC $\nu$-e elastic scattering (for Eqs. 62, 64, 65 and 66) (b) $\mathrm{CC} \nu_{e} e$ scattering (for Eq. 65), (c) $\mathrm{CC} \bar{\nu}_{e} e$ annihilation (for equation 66), and (d) inverse muon decay (for Eq. 64).

Table 4

\begin{tabular}{|c|c|c|c|}
\hline Reaction & $g_{L}$ & $g_{R}$ & $g_{L}^{2}+\frac{1}{3} g_{R}^{2}$ \\
\hline$\nu_{\mu} e^{-} \rightarrow \nu_{\mu} e^{-}$ & $-\frac{1}{2}+\sin ^{2} \theta_{W}$ & $\sin ^{2} \theta_{W}$ & 0.091 \\
$\bar{\nu}_{\mu} e^{-} \rightarrow \bar{\nu}_{\mu} e^{-}$ & $\sin ^{2} \theta_{W}$ & $-\frac{1}{2}+\sin ^{2} \theta_{W}$ & 0.077 \\
$\nu_{e} e^{-} \rightarrow \nu_{e} e^{-}$ & $\frac{1}{2}+\sin ^{2} \theta_{W}$ & $\sin ^{2} \theta_{W}$ & 0.551 \\
$\bar{\nu}_{e} e^{-} \rightarrow \bar{\nu}_{e} e^{-}$ & $\sin ^{2} \theta_{W}$ & $\frac{1}{2}+\sin ^{2} \theta_{W}$ & 0.231 \\
\hline
\end{tabular}

The coupling coefficients, $g_{L}$ and $g_{R}$, in Eq. 68 for the neutrino-electron scattering processes of Eqs. 62, 64, 65 and 66, respectively. The numerical values in the final column correspond to the combination of couplings that appears in the tree-level total cross section for $\sin ^{2} \theta_{W}=0.23$.

and the differential cross section for $\bar{\nu}_{e} e^{-} \rightarrow \bar{\nu}_{\mu} \mu^{-}$is

$$
\frac{d \sigma}{d y}\left(\bar{\nu}_{e} e^{-} \rightarrow \bar{\nu}_{\mu} \mu^{-}\right)=\frac{G_{F}^{2}\left(s-m_{\mu}^{2}\right)}{4 \pi}\left[\frac{s}{s-m_{\mu}^{2}} y(1-y)-\frac{m_{\mu}^{2}}{s}\right] .
$$




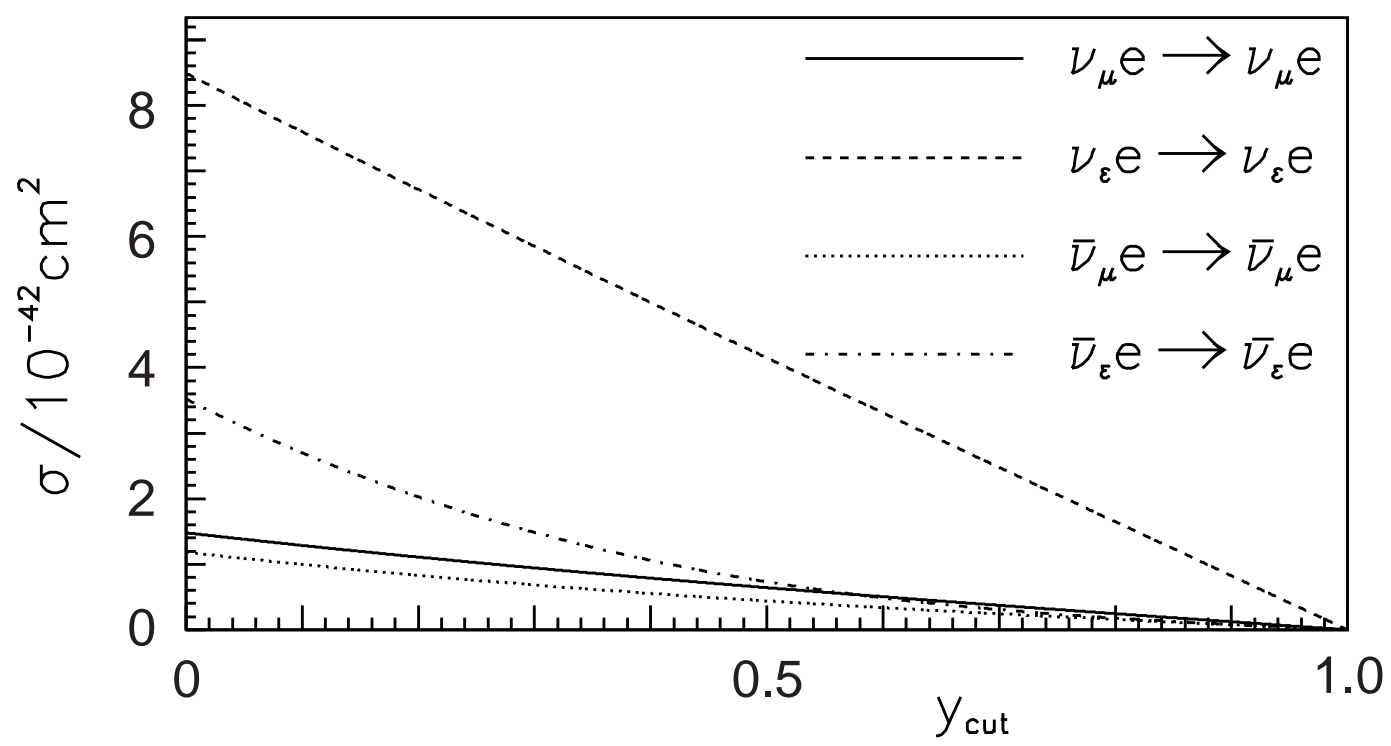

Fig. 9. Integral cross section for neutrino-electron scattering processes above any chosen cut on the inelasticity variable, $y>y_{\text {cut }}$, and assuming $E_{\nu}=30 \mathrm{GeV}$.

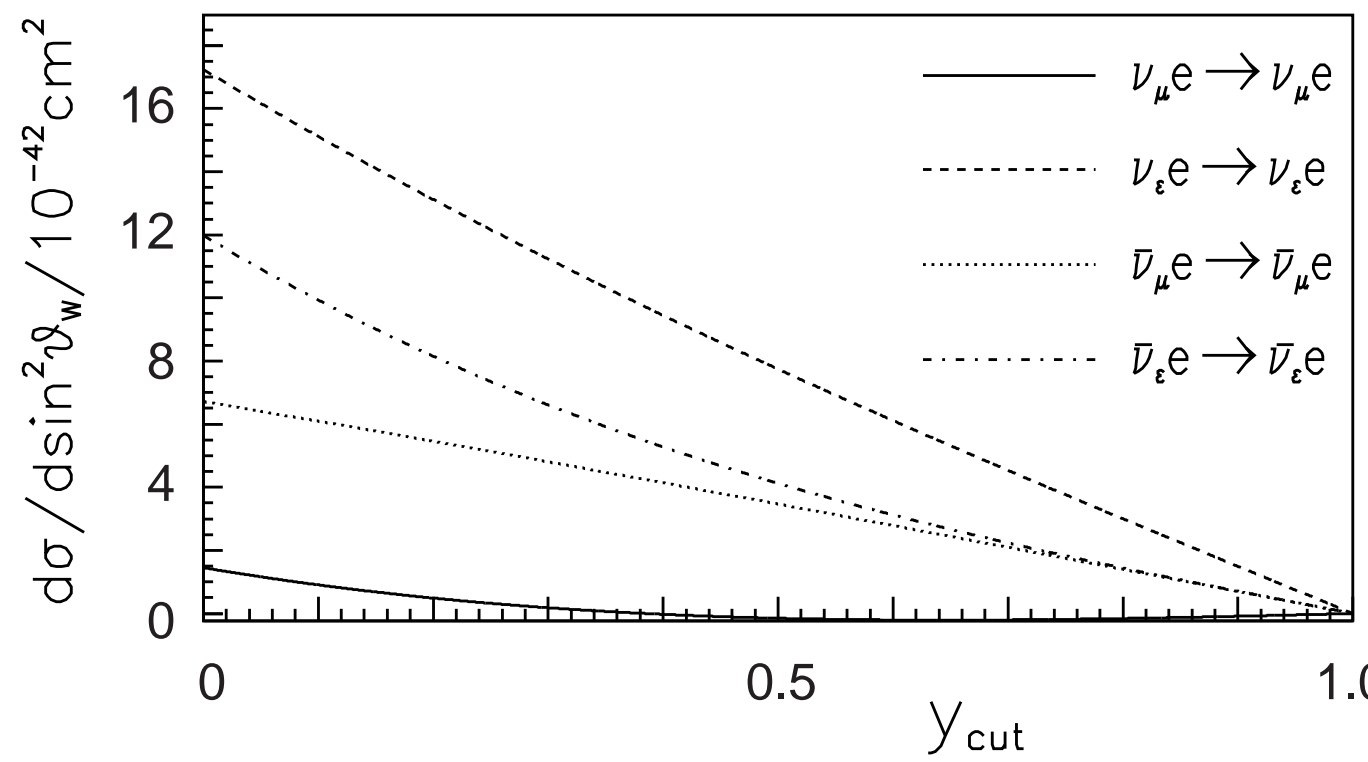

Fig. 10. Change in the integral cross section with respect to $\sin ^{2} \theta_{W}$ for neutrino-electron scattering processes above $y>y_{\text {cut }}$, assuming $E_{\nu}=30 \mathrm{GeV}$.

\subsubsection{Current Measurements of $\sin ^{2} \theta_{W}$ from Neutrino-Electron Scattering}

The best measurement of neutrino-electron scattering to date was performed in the CHARM II experiment in the CERN Sp $\bar{p} \mathrm{~S}$ neutrino beam. The beam was predominantly $\nu_{\mu}$ and $\bar{\nu}_{\mu}$, with an event sample of 2000 events in each of the $\nu_{\mu}$ and $\bar{\nu}_{\mu}$ beams. This led to a measurement of the weak mixing angle of $[64]$

$$
\sin ^{2} \theta_{W}=0.2324 \pm 0.0058 \text { (stat) } \pm 0.0059 \text { (syst) }
$$


Not surprisingly, systematic errors primarily result from normalization and background uncertainties.

\subsubsection{Overview of the Measurement Technique at a $\nu M C$}

The signature for $\nu-e$ scattering is a single electron with very low transverse momentum with respect to the neutrino beam direction, $p_{t} \lesssim \sqrt{m_{e} E_{\nu}}$. Therefore, the measured quantity to be converted to a cross section is the number of observed events consisting of a forward-going electron track with no hadronic activity and with an energy above some defined threshold value, $E_{\text {cut }}$.

In order to convert the event count to a cross section, the detector efficiency must be determined, backgrounds must be estimated and subtracted, and the integrated neutrino flux must measured and/or calculated.

In the discussion that follows, it will be seen that the physics sensitivity, backgrounds and flux normalization procedures will all differ markedly between the $\nu_{\mu} \bar{\nu}_{e}$ and $\bar{\nu}_{\mu} \nu_{e}$ beams. Experimental runs with the latter beam will have a greater statistical sensitivity to $\sin ^{2} \theta_{W}$ but the $\nu_{\mu} \bar{\nu}_{e}$ beam will provide two experimental advantages: (1) the possibility of flux normalization using the muons produced from inverse muon decay and by $\bar{\nu}_{e}-e^{-}$annihilation processes; and (2) the background from quasi-elastic electron neutrino scattering produces positrons rather than electrons, which can potentially be distinguished from the signal electrons by determining their charge sign.

\subsubsection{Statistical Sensitivity}

The number of signal interactions is related to the cross section for the process, $\sigma\left(E_{\nu}\right)$, and to the neutrino flux through the fiducial volume of the target, $\Phi\left(E_{\nu}\right)$, through

$$
N_{\nu-e} \propto \int \theta\left(y E_{\nu}-E_{c u t}\right) \sigma\left(E_{\nu}\right) \Phi\left(E_{\nu}\right) d E_{\nu}
$$

where the theta function is zero (one) for an argument less than (greater than) zero and the proportionality factor is determined by the mass depth of the target.

Figure 9 shows the integral cross section for the reactions above $y>y_{\text {cut }}$, and Fig. 10 shows the change in the cross section above $y>y_{\text {cut }}$ as a function of $\sin ^{2} \theta_{W}$. (A value of $y_{\text {cut }}$ relatively close to zero should likely be attainable by using a dedicated detector for this analysis.)

The statistical sensitivity to $\sin ^{2} \theta_{W}$ in any given channel is proportional to $\sqrt{\sigma} /\left(d \sigma / d \sin ^{2} \theta_{W}\right)$, and is shown in Fig. 11. In a neutrino beam produced by 


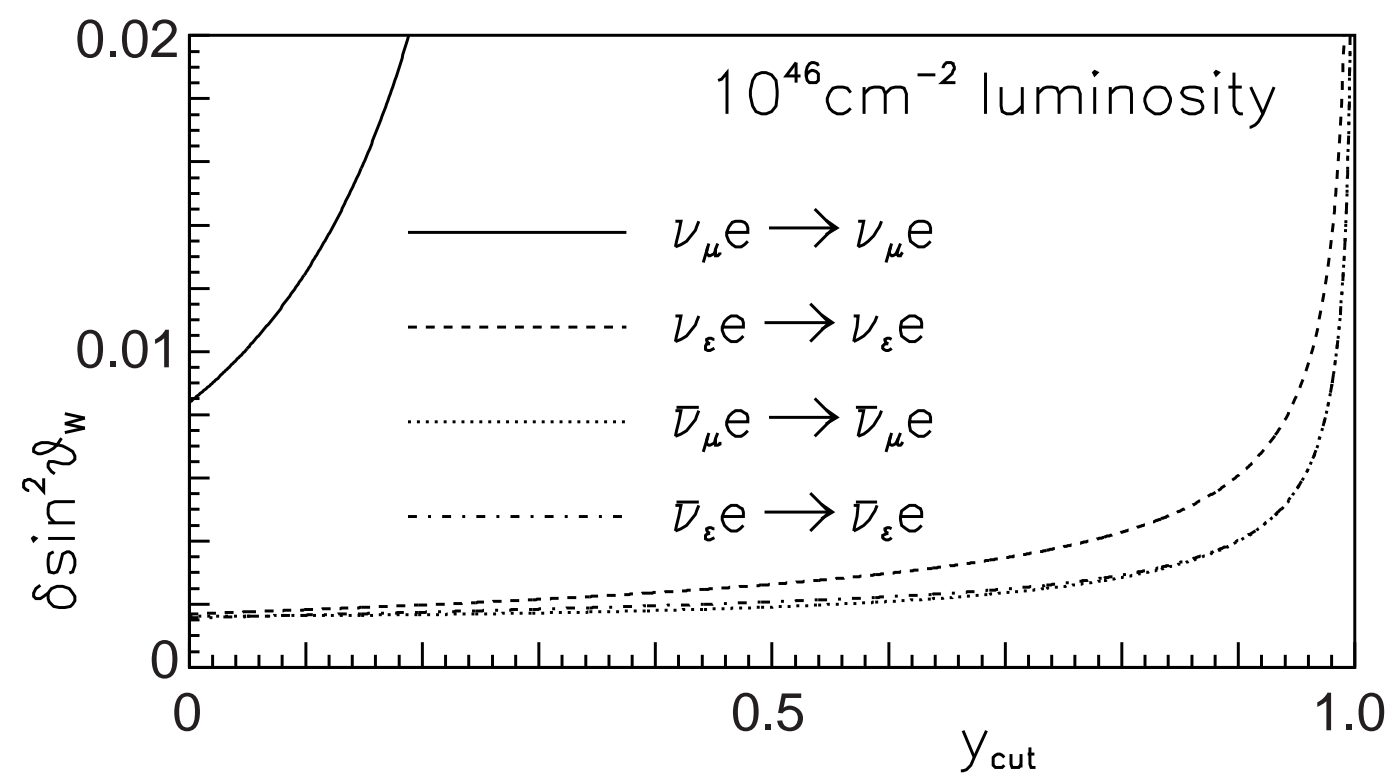

Fig. 11. Statistical uncertainty in $\sin ^{2} \theta_{W}$ for any chosen value of $y_{\text {cut }}$, from neutrino-electron scattering in beams of either $\nu_{\mu}, \nu_{e}, \bar{\nu}_{\mu}$ or $\bar{\nu}_{e}$. An integrated luminosity of $10^{46} \mathrm{~cm}^{-2}$ at a beam energy of $E_{\nu}=30 \mathrm{GeV}$ has been assumed. The values can be scaled to other neutrino energies by noting that the measurement's statistical uncertainty, for a given integrated neutrino flux through a specified detector, is proportional to the inverse square root of the average energy.

muon decays, the observed rate of visible electrons will include scattering of both neutrinos and antineutrinos.

Note that an undesirable feature of the $\mu^{-}$beam for measuring $\sin ^{2} \theta_{W}$ is that the dependences of the integral cross sections on $\sin ^{2} \theta_{W}$ for $\nu_{\mu}$ and $\bar{\nu}_{e}$ have opposite signs. The resulting sensitivity in integral cross sections for beams from $\mu^{ \pm}$decay is shown in Fig. 12, using the adequate approximation that the muon and electron type neutrino fluxes are assumed equal.

As an aside for a neutrino beam from a polarized muon beam, the exact sensitivity for this sort of summed measurement would depend on beam polarization, particularly for the $\mu^{-}$beam. Due to potential uncertainties in measuring the level of any muon beam polarization, better measurements of $\sin ^{2} \theta_{W}$ may well be obtained from muon beams where the polarization is identically zero or can at least be shown to average to zero over the course of a fill.

\subsubsection{Detector Design and Background Rejection}

The best types of detectors for detecting the signal of low $p_{t}$ single electrons are likely to be based around kiloton-scale active targets with inherent tracking capabilities and a high-rate capability. In order to minimize the level of confusion between the signal process and background events with $\gamma \rightarrow e^{-} e^{+}$, 


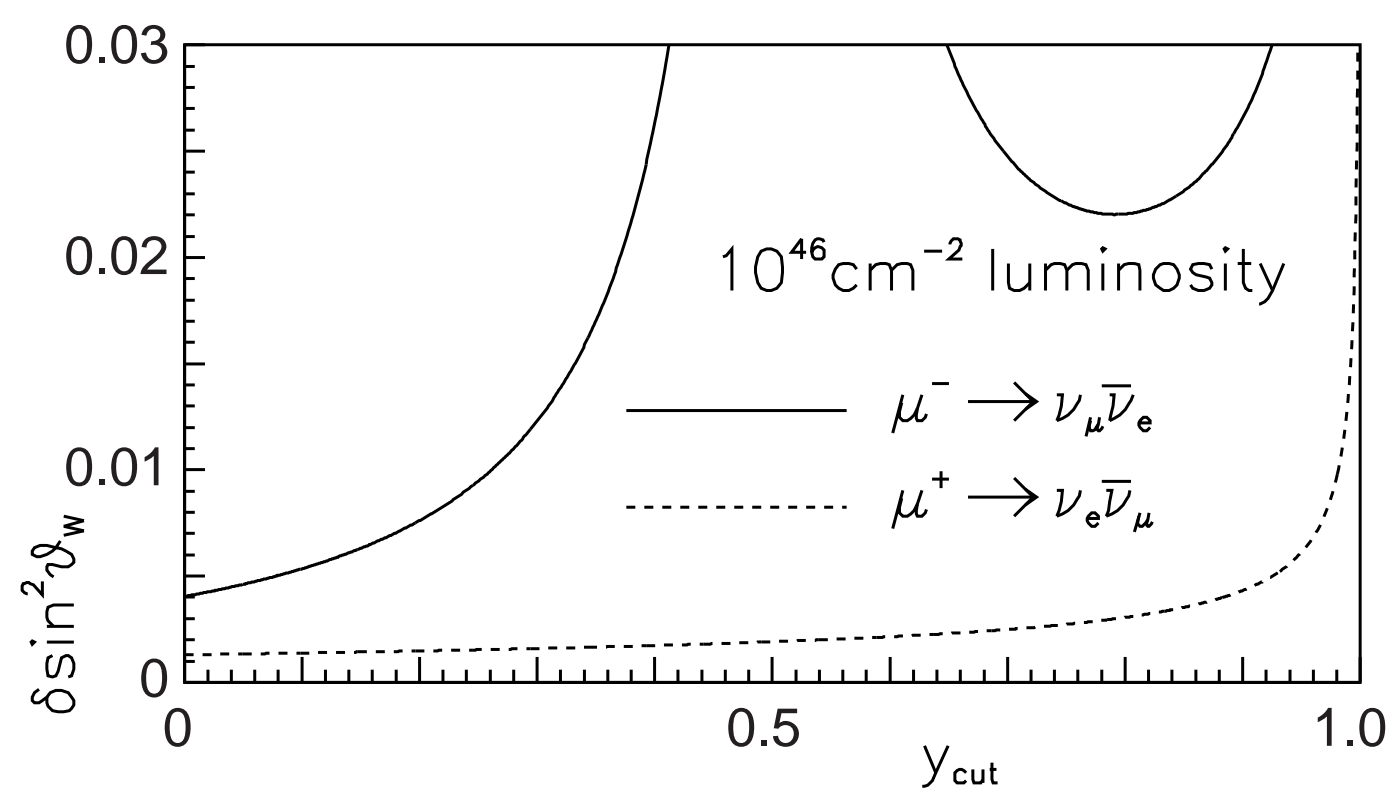

Fig. 12. Same as Fig. 11 except the event statistics has been summed over the experimentally indistinguishable contributions from the two neutrino components in the $\mu^{+}$-induced $\bar{\nu}_{\mu} \nu_{e}$ beams and the $\mu^{-}$-induced $\nu_{\mu} \bar{\nu}_{e}$ beams produced from muon storage rings.

the target should be composed of only low-Z elements in order to maximize the radiation length, and should contain very little dead material. The incorporation of a magnetic field to identify the lepton charge would further be helpful so as to reduce backgrounds from $\bar{\nu}_{e}$ charged current interactions, and a lepton charge measurement would also provide a cross-check of sign-symmetric detector backgrounds, such as $\gamma \rightarrow e^{+} e^{-}$.

For a detector with all these capabilities, rare low $p_{t}$ backgrounds such as coherent single $\pi^{0}$ production, which were a significant problem in the high-mass CHARM II neutrino detector[64], should not be difficult to identify and/or subtract on a statistical basis.

In order to also remove quasi-elastic $\nu_{e} N$ scattering backgrounds, which cannot be separated by electron charge sign identification in the $\bar{\nu}_{\mu} \nu_{e}$ beam, the detector will need to be capable of resolving the different $p_{t}$ distributions: $\nu_{e}$ quasi-elastic scattering off nucleons has a characteristic $p_{t}$ scale of $\sqrt{m_{N} E_{\nu}}$, i.e. larger by a factor of $\sqrt{m_{N} / m_{e}} \simeq 43$ than the signal process. In this case, signal and background suppression can be achieved by fitting the observed single electron $p_{t}$ distribution, which therefore must be measured with a $p_{t}$ resolution much better than $\sqrt{m_{N} E_{\nu}}$. To give a numerical example, the quasielastic cross section off nucleons at $E_{\nu}=30 \mathrm{GeV}$ on an isoscalar target is approximately 5 times greater than the inverse muon decay cross section and, in this case, a $p_{t}$ cut at $100 \mathrm{MeV}$ would leave a well-measured background of about $10 \%$ under the inverse muon decay peak. 


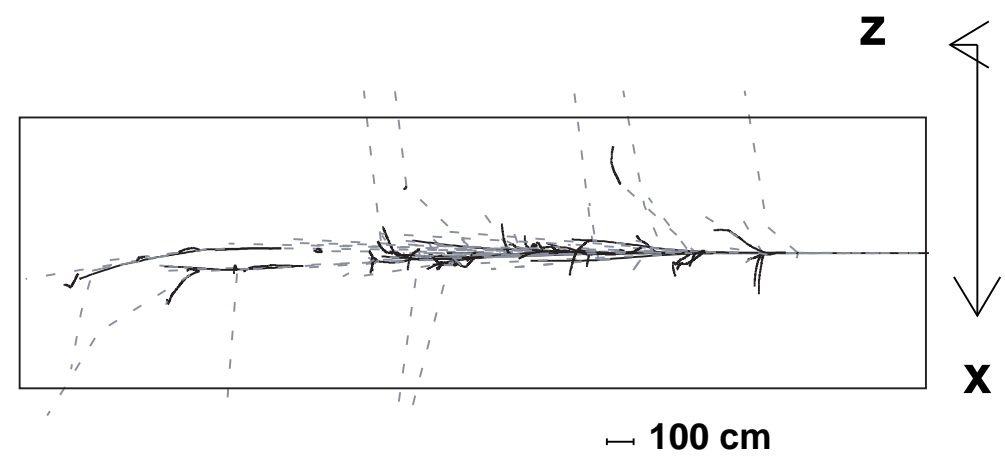

Fig. 13. Monte-carlo generated simulation of a high energy neutrino-electron scattering interaction in liquid helium. The distorted scale is indicated by the 10:1 ratio in the relative lengths of the $\mathrm{x}$ and $\mathrm{z}$ axes. The solid lines are electrons and positrons and the dashed lines are photons that would not be seen in the detector. The view is perpendicular to both the beam direction and to a 0.1 Tesla magnetic field. See text for further details.

These demanding and specialized requirements suggest a using dedicated detector rather than, e.g., the general purpose detector of Fig. 2. A natural choice is a time projection chamber (TPC) filled with a one or other of several candidate low-Z liquids.

A TPC using the lowest-Z element, liquid hydrogen, may unfortunately be ruled out because of insufficient electron mobility, although the possibilities for liquid hydrogen TPCs are again attracting some attention [65]. Liquid helium also suffers from poor mobility and potentially difficult operation because it lacks the ability to self-quench; however, it deserves further consideration because it has a radiation length of $7.55 \mathrm{~m}$, allowing very well resolved events.

Fig. 13 gives an example of a Monte Carlo-generated event in liquid helium. The interaction occurs at mid-height at the right-hand side of the figure and, in this typical case, the primary electron track travels easily sufficient distance to establish its initial vertex, direction, sign and the absence of extra tracks emerging from the vertex. Since the event is contained, the primary electron's energy could also be cross-checked calimetrically.

Liquid methane appears to be another good candidate for the TPC medium as its favorable electron transport properties have led to it being suggested for TPC detectors of up to several kilotons [66]. It is liquid at atmospheric pressure between -182.5 and -161.5 degrees centigrade and has a density of $0.717 \mathrm{~g} / \mathrm{cm}^{3}$ and a radiation length of $65 \mathrm{~cm}$. Heavier alkanes that are liquid at room temperature, such as octane, would be superior for safety and convenience if their electron transport properties were found to be acceptable.

Even if most DIS events are trivial to distinguish from the signal events, the detector must still be able to cope with the high interaction rate from 
the dominant background of DIS neutrino-nucleon interactions, which has a cross section three orders of magnitude larger than the signal processes. This problem is made even worse for the long drift times typical of the TPC geometry because the interactions from up to hundreds of turns (depending on experimental details) may pile up in the TPC read-out. This suggests the need for additional fast read-out of the events, which could plausibly come from, e.g., planes of scintillating fibers within the TPC volume.

Clearly, any such detector for the neutrino-electron scattering analysis must satisfy very stringent experimental requirements and its design and construction will be major projects.

\subsubsection{Flux Normalization for Neutrino-Electron Elastic Scattering}

Normalization of the cross section is also a significant issue since this probe of weak couplings is only as good as the normalization of the beam flux.

Normalization to the muon beam flux itself is a possibility which would work for both $\nu_{\mu} \bar{\nu}_{e}$ and $\bar{\nu}_{\mu} \nu_{e}$ beams. Theoretical predictions of the decay process would likely not limit this normalization technique. Instead, the ultimate accuracy should depend on uncertainties in the measured number of muons in the ring and on the beam beam dynamics, such as spot sizes, orbits, divergences and polarization if this doesn't average to zero. It would require a detailed analysis for a precisely specified muon ring design to determine whether or not muon beam measurements and modeling could predict $\nu \mathrm{MC}$ neutrino fluxes at the $10^{-4}$ level required to be useful for this analysis.

For the $\nu_{\mu} \bar{\nu}_{e}$ beam, an alternative candidate for normalization is single muon production in neutrino-electron scattering through the processes of Eqs. 63 and 67. Like the signal, the absolute cross sections for these normalization processes are also extremely accurately predictable but with the crucial difference that they don't depend on $\sin ^{2} \theta_{W}$.

It is conceivable that this absolute normalization in the $\nu_{\mu} \bar{\nu}_{e}$ beam could then also be transferred to the $\bar{\nu}_{\mu} \nu_{e}$ beam by using the ratio of quasi-elastic events to provide a relative normalization between the two beam types.

\subsubsection{Sensitivity to New Physics Processes}

For a $\mu^{-}$beam, the cross section calculations above show that, if the very challenging experimental systematic uncertainties can be satisfactorily addressed then sensitivities of approximately $\delta \sin ^{2} \theta_{W} \sim 0.0007$ would be reached for an integrated luminosity of $10^{46} \mathrm{~cm}^{-2}$ and a mean neutrino beam energy of $30 \mathrm{GeV}$, which corresponds to approximately $1.5 \times 10^{9}$ DIS charged current 
events (c.f. Table 2).

The $\mu^{+}$beam's statistical sensitivity would be about a factor of three better, with the caveat that beam flux normalization and experimental backgrounds are both even more challenging. With an integrated luminosity of $10^{46} \mathrm{~cm}^{-2}$, expected event sample sizes would be approximately $1.5 \times 10^{6}$ for a $\mu^{-}$beam and $3 \times 10^{6}$ for a $\mu^{+}$beam. Normalization and background uncertainties must therefore be kept at the few times $10^{-4}$ level in order to achieve this precision.

Such a measurement could be used to probe for hints of physics beyond the Standard Model by, for example, interpreting it in terms of a sensitivity to a high mass contact interaction with a Lagrangian of the form

$$
\mathcal{L}=\sum_{H \in\{L, R\}} \frac{ \pm 4 \pi}{\Lambda_{H_{e} H_{\nu}}^{ \pm}}\left(\Lambda_{H_{e} H_{\nu}}^{ \pm}\right) \bar{e}_{H_{e}} \gamma^{\mu} e_{H_{e}} \bar{\nu}_{H_{\nu}} \gamma_{\mu} \nu_{H_{\nu}}
$$

where the $H$ indices represent helicity states of the electron and neutrino. The statistics given above would probe contact interactions at mass scales $\Lambda \sim 10 \mathrm{TeV}$, again assuming that statistical uncertainties dominate.

\section{3 $\sin ^{2} \theta_{W}$ from Deep Inelastic Scattering}

Measurements of $\sin ^{2} \theta_{W}$ in neutrino-nucleon DIS experiments, using the neutrino beams that have been available from $\pi / K$ decays, have already provided an excellent testing ground for the Standard Model. Nucleons, however, make for a most unappealing and difficult target, and it is necessary to consider ratios of observable processes in order to make sense of the results.

\subsubsection{Previous Measurements}

The CCFR $\nu$ experiment extracted $\sin ^{2} \theta_{W}[67,68]$ through a measurement of the ratio of the cross sections for $\mathrm{NC}$ and $\mathrm{CC}$ interactions, as expressed in the Llewellyn-Smith formula [69] :

$$
R^{\nu(\bar{\nu})}=\frac{\sigma_{N C}^{\nu(\bar{\nu})}}{\sigma_{C C}^{\nu(\bar{\nu})}}=\rho^{2}\left(\frac{1}{2}-\sin ^{2} \theta_{W}+\frac{5}{9} \sin ^{4} \theta_{W}\left(1+\frac{\sigma_{C C}^{\bar{\nu}(\nu)}}{\sigma_{C C}^{\nu(\bar{\nu})}}\right)\right)
$$

where the value of the parameter $\rho$ depends on the nature of the Higgs sector and has the value $\rho=1$ in the Standard Model. This method, although it removed much of the uncertainty due to QCD effects in the target, does leave some rather large uncertainties associated with heavy quark production from the quark sea of the nucleon target. 
The successor experiment, NuTeV (FNAL-E815), has improved upon the CCFR measurement by using separate neutrino and antineutrino beams. Separation of neutrino and antineutrino neutral current events allows the utilization of the Paschos-Wolfenstein relationship [70]:

$$
R^{-}=\frac{\sigma_{N C}^{\nu}-\sigma_{N C}^{\bar{\nu}}}{\sigma_{C C}^{\nu}-\sigma_{C C}^{\bar{\nu}}}=\frac{R^{\nu}-r R^{\bar{\nu}}}{1-r}=\rho^{2}\left(\frac{1}{2}-\sin ^{2} \theta_{W}\right)
$$

where

$$
r=\frac{\sigma(\bar{\nu}, C C)}{\sigma(\nu, C C)} \simeq 0.5
$$

$R^{-}$is, by construction, sensitive only to scattering from valence quarks in the proton, and this considerably reduces the theoretical uncertainties associated with the target. NuTeV has presented a preliminary result[71] of

$$
\sin ^{2} \theta_{W}=0.2253 \pm 0.0019(\text { stat }) \pm 0.0010 \text { (syst) }
$$

There were two dominant systematic uncertainties in the CCFR experiment: 1) $\nu_{e}$ flux and 2) CC charm production. These two major systematic uncertainties in CCFR were reduced in the $\mathrm{NuTeV}$ experiment by utilizing signselected neutrino beams, leaving event statistics as the dominant remaining uncertainty.

These past neutrino fixed target experiments used dense calorimetric neutrino targets in order to increase the interaction rate, and such targets did not allow one to distinguish between electron-neutrino-induced charged current interactions (CC) and neutral current (NC) interactions. Such experimental set-ups would be fatal for $\nu \mathrm{MC}$ analyses with 2-component $\nu_{\mu} \bar{\nu}_{e}$ and $\bar{\nu}_{\mu} \nu_{e}$ beams and, as will be discussed further; a high performance tracking target such as that in Fig. 2 is instead indicated.

\subsubsection{The Experimental Extraction of $\sin ^{2} \theta_{W}$}

As in previous neutrino experiments, the measured quantity used to determine $\sin ^{2} \theta_{W}$ at $\nu \mathrm{MCs}$ will be a ratio of NC to CC DIS events. However, the NuTeVstyle ratio of Eq. $76, R^{-}$, will not be accessible in the 2 -component beams at $\nu \mathrm{MCs}$ because NC events from neutrinos and those from antineutrinos will not be distinguishable on an event-by-event basis. Instead, the experimentally accessible NC-to-CC event ratios for both the $\nu_{\mu} \bar{\nu}_{e}$ and $\bar{\nu}_{\mu} \nu_{e}$ beams essentially correspond to linear combinations of the Llewellyn-Smith ratios for neutrinos and antineutrinos that were given in equation 75 . 


\begin{tabular}{|c|c|c|}
\hline SOURCE OF UNCERTAINTY & NuTeV & $\nu \mathrm{MC}$ \\
\hline DATA STATISTICS & 0.00190 & $<0.0001$ \\
\hline$\nu_{e}$ flux Modeling & 0.00045 & irrelevant \\
\hline Transverse Vertex Position & 0.00040 & negligible \\
\hline Event Energy Measurements & 0.0051 & irrelevant \\
\hline Event Length & 0.0037 & irrelevant \\
\hline Primary lepton ID & N.A. & $<0.00020(?)$ \\
\hline TOTAL EXP. SYST. & 0.00078 & negligible(?) \\
\hline Charm Production & negligible & $<0.00030$ \\
\hline Higher Twist & 0.00011 & $<0.00011$ \\
\hline Longitudinal Cross Section & 0.00004 & negligible \\
\hline Charm Sea, $( \pm 100 \%)$ & 0.00002 & negligible \\
\hline$\sigma^{\bar{\nu}} / \sigma^{\nu}$ & 0.00021 & negligible \\
\hline Non-Isoscalar Target & 0.00017 & $>0.00010$ \\
\hline Structure Functions & 0.00010 & negligible \\
\hline Rad. Corrections & 0.00051 & $<0.00050$ \\
\hline TOTAL PHYSICS MODEL & $\sim 0.00069$ & $<0.00050$ \\
\hline "TOTAL UNCERTAINTY & 0.00220 & $<0.00050$ \\
\hline Equivalent $\Delta M_{W}$ & $110 \mathrm{MeV} / \mathrm{c}^{2}$ & $<25 \mathrm{MeV} / \mathrm{c}^{2}$ \\
\hline
\end{tabular}

Table 5

Estimates of $\sin ^{2} \theta_{W}$ uncertainties in a $\nu \mathrm{MC}$ analysis, compared to those for the $\mathrm{NuTeV}$ preliminary result [71]. See the text for details on how the estimated uncertainties from $\nu \mathrm{MCs}$ were arrived at.

The relevant ratios for $\nu \mathrm{MCs}$ will be:

$$
R_{\mu^{-}}=\frac{\sigma\left(\nu_{\mu}, N C\right)+\sigma\left(\bar{\nu}_{e}, N C\right)}{\sigma\left(\nu_{\mu}, C C\right)+\sigma\left(\bar{\nu}_{e}, C C\right)}=\frac{R_{\nu}+g r R_{\bar{\nu}}}{1+g r}
$$

for the $\nu_{\mu} \bar{\nu}_{e}$ beam, and

$$
R_{\mu^{+}}=\frac{\sigma\left(\bar{\nu}_{\mu}, N C\right)+\sigma\left(\nu_{e}, N C\right)}{\sigma\left(\bar{\nu}_{\mu}, C C\right)+\sigma\left(\nu_{e}, C C\right)}=\frac{R_{\nu}+g^{-1} r R_{\bar{\nu}}}{1+g^{-1} r}
$$

for the $\bar{\nu}_{\mu} \nu_{e}$ beam, with $r$ previously defined in Eq. 77 and $g$ the energyweighted flux ratio between $\bar{\nu}_{e}$ and $\nu_{\mu}$ in a $\nu_{\mu} \bar{\nu}_{e}$ beam or - equivalently for a 
non-polarized beam - between $\nu_{e}$ and $\bar{\nu}_{\mu}$ in a $\bar{\nu}_{\mu} \nu_{e}$ beam:

$$
g \equiv \frac{<x>^{e}}{<x>^{\mu}}=\frac{\int \Phi\left(E_{\bar{\nu}_{e}}\right) E_{\bar{\nu}_{e}} d E_{\bar{\nu}_{e}}}{\int \Phi\left(E_{\nu_{\mu}}\right) E_{\nu_{\mu}} d E_{\nu_{\mu}}}=\frac{\int \Phi\left(E_{\nu_{e}}\right) E_{\nu_{e}} d E_{\nu_{e}}}{\int \Phi\left(E_{\bar{\nu}_{\mu}}\right) E_{\bar{\nu}_{\mu}} d E_{\bar{\nu}_{\mu}}} .
$$

Equations 79 and 80 have made use of lepton universality, which implies that $\nu_{e} N$ scattering cross sections become equal to those for $\nu_{\mu} N$ at energy scales well above the electron and muon masses. The second of the two equations differs from the first only in the replacement of $g$ by $g^{-1}$.

An analytic calculation [9] gives the value $g=6 / 7$ for the neutrino beam produced from an idealized pencil beam of unpolarized muons. It follows that the numerical values of the measurements from the $\nu_{\mu} \bar{\nu}_{e}$ and $\bar{\nu}_{\mu} \nu_{e}$ beams will be nearly identical:

$$
R_{\mu^{-}} \simeq 0.330 ; \quad R_{\mu^{+}} \simeq 0.332
$$

where we have used the predictions from Eq. 75 of $R^{\nu}=0.317$ and $R^{\bar{\nu}}=0.359$ for $\sin ^{2} \theta_{W}=0.225$. Of more experimental relevance, the statistical sensitivities to $\sin ^{2} \theta_{W}$ are also nearly identical, as is indicated by the logarithmic derivatives:

$$
\frac{1}{R_{\mu^{-}}} \frac{d R_{\mu^{-}}}{d \sin ^{2} \theta_{W}}=-1.55 ; \quad \frac{1}{R_{\mu^{+}}} \frac{d R_{\mu^{+}}}{d \sin ^{2} \theta_{W}}=-1.47 .
$$

The numerical similarities between the complementary variables $R_{\mu^{-}}$and $R_{\mu^{+}}$, from $\nu_{\mu} \bar{\nu}_{e}$ and $\bar{\nu}_{\mu} \nu_{e}$ beams respectively, mean that the two measurements can be regarded as nearly identical from a physics standpoint but with slightly different experimental systematics due to the approximate interchange of electron and muon energy spectra in the CC final states.

Because of the different kinematics for neutrino vs. antineutrino interactions, the CC event sample from the $\nu_{\mu} \bar{\nu}_{e}$ beam will contain a softer spectrum of primary muons than electrons and vice versa for the $\bar{\nu}_{\mu} \nu_{e}$ beam. The comparison of two theoretically similar measurements with different experimental challenges will be a valuable cross-check on the analyses. In this respect, it is helpful that muon storage rings are likely [9] to have the capability to reverse the polarity of the ring to choose between $\nu_{\mu} \bar{\nu}_{e}$ or $\bar{\nu}_{\mu} \nu_{e}$ beams at any given time.

\subsubsection{Detector Requirements}

Any $\nu N$ DIS measurements of $\sin ^{2} \theta_{W}$ at a $\nu \mathrm{MC}$ would be expected to be a large experimental extrapolation from today's measurements. The most demanding requirement on the detector for this analysis will be the ability to 
efficiently distinguish $\mathrm{CC}$ events, with their primary electrons or muons, from the purely hadronic events produced in $\mathrm{NC}$ interactions.

The large component of electron (anti)neutrinos in both the $\bar{\nu}_{\mu} \nu_{e}$ and $\nu_{\mu} \bar{\nu}_{e}$ beams at $\nu \mathrm{MCs}$ rules out use of traditional calorimetric neutrino target/detectors since these cannot easily distinguish $\nu_{e}$-induced $\mathrm{CC}$ interactions from $\mathrm{NC}$ interactions.

In contrast, a CCD-based tracking target and general purpose detector such as that of Fig. 2 appears to be well suited to the requirements for this analysis because of its expected good performance [9] in identifying both primary muons and electrons and its further ability to control backgrounds from secondary electrons or background muons from pion decays that fake primary leptons. Even so, it might even be helpful if the electron identification capabilities of such a detector were further bolstered by incorporating transition radiation detectors directly downstream from the tracking detectors.

\subsubsection{Estimated Uncertainties}

Table 5 displays a comparison between the uncertainties in the $\nu N$ DIS measurements of $\sin ^{2} \theta_{W}$ from today's most precise measurement [71] and rough estimates of the corresponding uncertainties from a $\nu \mathrm{MC}$ measurement, which will now be discussed in turn.

The statistical uncertainty from the roughly one million events at the $\mathrm{NuTeV}$ experiment was the largest uncertainty in that $\sin ^{2} \theta_{W}$ analysis. Table 2 suggests $\nu \mathrm{MC}$ event statistics of $10^{9}$ events or more, corresponding to a reduction in statistical uncertainty by at least a factor of 30 and pushing the absolute statistical uncertainty in $\sin ^{2} \theta_{W}$ to below 0.0001 .

Turning now to experimental uncertainties, the NuTeV uncertainty of 0.0004 due to $\nu_{e}$ flux was relevant only for calorimetric neutrino targets and will no longer exist for the tracking target/detectors discussed for $\nu \mathrm{MCs}$ that will be capable of distinguishing, on an event-by-event basis, between NC interactions and the $\mathrm{CC}$ interactions of $\nu_{e}$ 's. Uncertainties from energy scale and event length in the $\mathrm{NuTeV}$ analysis will also be irrelevant for $\nu \mathrm{MC}$ detectors because they were associated with NuTeV's statistical event-length method of separating NC from CC events.

The improved $\nu \mathrm{MC}$ method of identifying primary leptons on an event-byevent basis will instead have to contend with uncertainties in the identification efficiencies for the primary leptons that distinguish CC from NC events. Every misidentification moves that event between the numerator and denominator of the experimental ratios of Eqs. 79 and 80, so it is clear that the fractional uncertainty in the level of misidentifications must be reduced to well below 
the $10^{-3}$ magnitude desired for the fractional uncertainty in $\sin ^{2} \theta_{W}$. Some confidence that this might be achievable comes from the very high lepton identification efficiencies for the detector scenario discussed above.

Both the rejection of backgrounds and the positive identification of the primary lepton are generally more difficult for low energy leptons, so the $\sin ^{2} \theta_{W}$ measurement would benefit from using cuts on the minimum lepton energy. The value of this energy cut must be balanced against increasing theoretical uncertainties as progressively more of the event sample is cut away. In any case, estimation of the identification efficiency for primary leptons may well be the largest experimental uncertainty in a measurement dominated by theoretical uncertainties.

An improved understanding of several potential theoretical uncertainties will be required to attain a $\sin ^{2} \theta_{W}$ measurement that could be meaningfully interpreted as equivalent to a sub-25 $\mathrm{MeV} W$ mass uncertainty. In particular, calculations and/or measurements to minimize the charm production uncertainty, higher twist effects, radiative corrections, and uncertainties in the longitudinal structure function $R_{L}$ will need to be dealt with.

In this respect, it is helpful that several of the theoretical uncertainties can be calibrated using the same $\nu \mathrm{MC}$ event sample as used for the $\sin ^{2} \theta_{W}$ analysis. Hence, the enormous increase in statistical power of $\nu \mathrm{MCs}$ over today's neutrino experiments should also help to minimize some of the systematic uncertainties in Table 5.

Good examples of theoretical uncertainties that are amenable to experimental calibration are the large uncertainty due to charm mass effects and the related uncertainties in estimating the charm and strange seas of the nucleons. A Nextto-Leading-Order (NLO) fit to the charm mass from CCFR [17] obtained $m_{c}=$ $1.71 \pm 0.19 \pm 0.02 \mathrm{GeV} / c^{2}$, corresponding to a charm production uncertainty in the CCFR $\sin ^{2} \theta_{W}$ measurement of $\delta \sin ^{2} \theta_{W}=0.003$. Since the statistics at a $\nu \mathrm{MC}$ might be three or more orders of magnitude larger than in the CCFR experiment, Table 5 somewhat arbitrarily chooses an improvement by a factor of 10 on the CCFR uncertainty. This is less improvement than the factor of 30 or more that would be predicted from straightforward statistical scaling but a careful analysis would be required to establish the actual level at which residual theoretical uncertainties set in that cannot be calibrated away using the experimental data.

Strange quark mass effects in $\nu_{\ell} \bar{u} \rightarrow \ell^{-} \bar{s}$ and $\nu_{\ell} s \rightarrow \nu_{\ell} s$ provide a much smaller theoretical effect that fell below the uncertainty threshold for the $\mathrm{NuTeV}$ analysis but whose corrections and uncertainties would need to be checked for a $\nu \mathrm{MC}$ analysis. Although presumably a very small correction, its effects are difficult to reliably establish because lattice gauge calculations predict that 
$m_{s}$ has an awkward value: $m_{s} \simeq 300 \mathrm{MeV} \simeq \Lambda_{Q C D}$.

Higher twist effects are assessed as one of the larger theoretical uncertainties in today's measurements. However, a recent study [72] indicates that most of the higher twist effects might be able to be reinterpreted as higher order QCD corrections that can be determined from the structure functions measured in the $\nu \mathrm{MC}$ data sample. For the theoretical precision required at a $\nu \mathrm{MC}$ measurement, it may be necessary to evaluate and correct for the residual small effects from "radiative higher twist processes" such as $\nu_{\ell} n \rightarrow \gamma \ell^{-} p$. The radiative photon present in $\mathrm{CC}$ events generates a $\mathrm{CC} / \mathrm{NC}$ asymmetry and can boost the apparent $Q^{2}$ of events to high enough values to evade cuts designed to suppress higher twist corrections.

As was already mentioned in Sec. 2, cancellations of theoretical uncertainties by applying isospin invariance relations are very important for reducing the uncertainties in $\sin ^{2} \theta_{W}$ and in other analyses in $\nu N$ DIS experiments and this is the motivation for using neutrino targets that are approximately isoscalar. This theoretical handle was useful for both the CCFR and $\mathrm{NuTeV}$ analyses, using a neutrino target detector with a neutron excess of $(N-Z) /(N+Z)=$ $0.0567 \pm 0.0005$. Isospin invariance relations should be even more applicable for, e.g., a CCD target at a $\nu \mathrm{MC}$ since the silicon substrate of CCD's has a neutron excess of only $0.3 \%$ and even the residual target components will have small non-isoscalarities: aluminum conductor has a neutron excess of $3.6 \%$ and the dominant carbon component of the support structure has an excess of less than $0.1 \%$.

Indeed, the $\nu \mathrm{MC}$ neutrino target might be sufficiently isoscalar that uncertainties due to the neutron excess might fall below those due to nucleon isospinviolating effects[73] arising from electromagnetic and quark-mass contributions that break the generally assumed isospin invariance relations between the up and down quarks in protons and neutrons: $u_{p}\left(x, Q^{2}\right)=d_{n}\left(x, Q^{2}\right)$ and $u_{n}\left(x, Q^{2}\right)=d_{p}\left(x, Q^{2}\right)$. Such effects will be present even in a deuterium target [9] or other pure isoscalar targets.

The theoretical uncertainty due to electroweak radiative corrections in the $\mathrm{NuTeV}$ experiment, $\delta \sin ^{2} \theta_{W}=0.00051$, deserves further theoretical attention for a $\nu \mathrm{MC}$ since it does not appear to be amenable to data-based reductions from the improved statistics and experimental conditions at a $\nu \mathrm{MC}$ and so it might well dominate the total uncertainty at a $\nu \mathrm{MC}$ if the theoretical calculations are not improved upon, as will now be discussed.

The current state of electroweak radiative corrections suffers from the fact that only one attempt at a complete calculation exists, by Bardin and Dokuchaeva[74], which, curiously, is unpublished. This calculation includes electroweak effects to one loop, is lengthy and complicated, and it would benefit from independent 
confirmation. Further, it has several theoretical shortcomings, as follows. The Bardin-Dokuchaeva result depends explicitly on quark masses, which introduces a spurious model dependence. Similar calculations of EW radiative corrections in $W$ mass production in $\bar{p} p$ annihilation show that quark mass effects can be absorbed into parton distributions and fragmentation functions[75]. The calculation is only approximately valid to leading log in QCD. In particular, it neglects scaling violation effects in important diagrams involving muon bremsstrahlung. It also neglects contributions from longitudinal partons and effects from the target mass. Further, it does not incorporate heavy quark effects in CC and NC charm production and, finally, it is not differential in the final state radiated photon momentum vector.

A $\nu \mathrm{MC}$ measurement of $\sin ^{2} \theta_{W}$ would benefit greatly from a new EW radiative correction program with the aim of reducing the residual error on the effective $W$ mass to $\pm 1 \mathrm{MeV}$. To reach this level, it may be necessary to re-sum large lepton logs, to include order $\alpha \alpha_{S}$ contributions, and to apply EM radiative corrections to input parton distribution function sets. Such a program should also provide the capability for the explicit generation of $\gamma \mu q$ final states.

\subsubsection{Comparisons of Expected Precisions from $\sin ^{2} \theta_{W}$ Measurements in Dif- ferent Experimental Processes}

To summarize the content of Table 5, the uncertainty on a $\nu \mathrm{MC}$ DIS measurement of $\sin ^{2} \theta_{W}$ might well be equivalent to on the order of a $25 \mathrm{MeV}$ uncertainty on the $W$ mass and could perhaps improve on this if the theoretical uncertainties due to radiative corrections can be controlled.

In order to put this in the context of collider measurements at the energy frontier, Fig. 14 adds the inferred $W$ mass information from a $\nu \mathrm{MC}$ determination of $\sin ^{2} \theta_{W}$ to a plot showing the expected status of collider $W$ and top quark mass measurements by the year 2010. Although the level of the bands and the actual slope of the curves might change depending on other parameters in the measurements, this figure gives a good idea of the level of accuracy one might expect.

The most precise measurement of $M_{W}$ by the year 2010 is expected to come from direct measurements at TeV33. The contour represents the $68 \%$ confidence level from the TeV33 expectations of $\delta M_{W}=20 \sim 30 \mathrm{MeV} / c^{2}$ and $\delta M_{t}=1 \sim 2 \mathrm{GeV} / c^{2}$, with $\int \mathcal{L} d t=10 \mathrm{fb}^{-1}$ and using the traditional $M_{T}$ method [76]. As can be seen in Fig. 14, since the errors from both the direct measurements and the $\nu \mathrm{MC}$ are going to be extremely small, and the $\nu \mathrm{MC}$ measurement provides the Standard Model-based band in $M_{W}-M_{T}$ plane, the measurements would be complementary to each other in testing the Standard Model by providing an independent prediction of the Standard Model 


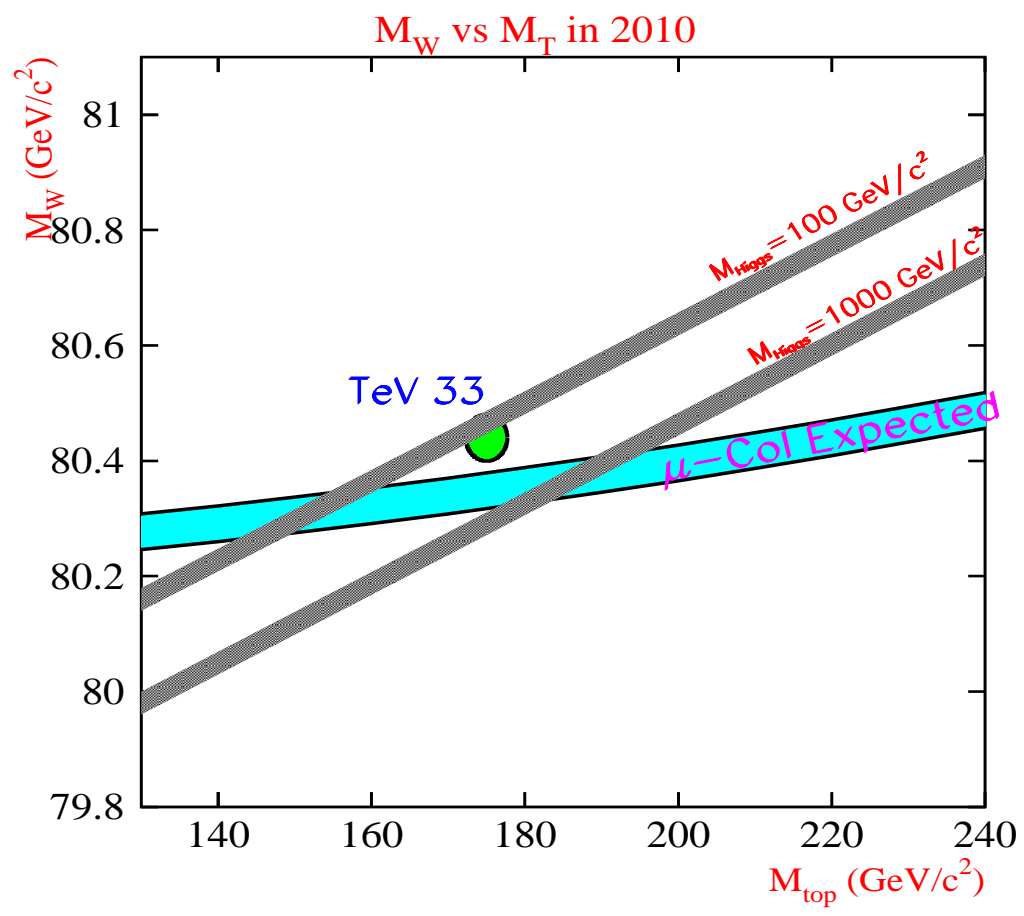

Fig. 14. Expected future restrictions in the size of the allowed regions in a plot of $W$ mass vs. top quark mass, following experiments at $\mathrm{TeV} 33$ and at a $\nu \mathrm{MC}$, as illustrated by the thicknesses of the bands and ellipse. The exact positions of the shaded regions are for illustrative purposes only.

Higgs mass to better than $\sim 20 \%$.

\subsection{Summary on $\sin ^{2} \theta_{W}$ Measurements at $\nu M C s$}

This section has demonstrated that precision tests of the electroweak section of the Standard Model can be expected to play an important part in the physics program of $\nu$ MCs. Two possible tests were discussed, one each from neutrino-nucleon scattering and neutrino-electron scattering.

The former process will allow tests of unparalleled statistical accuracy but will likely suffer from substantial QCD uncertainties and, perhaps, also from experimental systematic uncertainties. By contrast, neutrino-electron scattering may be a statistical challenge even at a $\nu \mathrm{MC}$, but it offers the possibility of a very clean measurement if experimental systematics associated with normalization and backgrounds can be well enough controlled by an appropriate design of the detector and analysis. 


\section{$5 \quad$ Rare and Exotic Processes}

Despite the impressive direct and indirect searches for new physics available at the higher center of mass energies at the Tevatron, LEP, HERA and LHC colliders, some searches remain unique to neutrino experiments and these could be improved quite significantly with the much higher event statistics and improved experimental conditions promised at $\nu \mathrm{MCs}$. This section presents several examples of such processes, emphasizing the complementarity of these studies to already existing programs.

Additionally, two rare processes, $\bar{\nu}_{e} e^{-}$annihilation and $W / Z-\gamma$ scattering, present novel tests of low energy features of the Standard Model.

\subsection{New Physics Sensitivity}

\subsubsection{Flavor Changing Neutral Currents}

Besides testing current predictions of the Standard Model, a $\nu \mathrm{MC}$ offers opportunities to search for new phenomena in yet unexplored physical regions. An example is neutral current production of a single heavy quark:

$$
\begin{aligned}
& \nu_{\mu} N \rightarrow \nu_{\mu} c X_{C=0}, \quad \nu_{\mu} N \rightarrow \nu_{\mu} \bar{c} X_{C=0}, \\
& \nu_{\mu} N \rightarrow \nu_{\mu} b X_{B=0}, \quad \nu_{\mu} N \rightarrow \nu_{\mu} \bar{b} X_{B=0},
\end{aligned}
$$

which would signal the presence of flavor changing neutral current (FCNC) processes. These reactions can provide important constraints on new physics as they occur in the Standard Model only at the one loop level while new physics effects can occur at both the tree level and one loop level. Examples of the former include new intermediate bosons with FCNC quark couplings while examples of the latter include a wide class of new physics models such as supersymmetry and technicolor. Some of these models are already constrained from other measurements[84]. Unfortunately, gains in sensitivity to this type of new physics increase only slowly with event statistics. For instance, in the models with new tree-level FCNC interactions, such as string-inspired models with neutral $Z^{\prime}$ bosons, the FCNC rate due to $Z^{\prime}$ exchange is proportional to $1 / M_{Z^{\prime}}^{4}$ and so sensitivity to $M_{Z^{\prime}}$ only improves at best as the fourth root of rate increases.

The FCNC vertices $\nu \nu s b$ that contribute to equation (83) will be extensively studied in exclusive and inclusive $B$ decays at the $B$ factories as well as at CERN [83]. ALEPH has obtained the best bound so far: $B R(B \rightarrow \nu \bar{\nu} X) \leq$ $7.7 \times 10^{-4}$. Within the Standard Model one predicts a value of $\sim 4 \times 10^{-5}$. In contrast, studies of the $\nu \nu d b$ vertex will be extremely challenging even at 
future $B$ factories. Processes that involve neutrinos, such as $B \rightarrow X_{s} \nu \bar{\nu}$ and $\nu_{\mu} N \rightarrow \nu_{\mu} b X_{B=0}$, have the considerable advantage over the corresponding FCNC processes involving charged leptons that their rates are mostly determined by short-distance physics, which ensures the robustness of the theoretical predictions.

, in the event of any observed signal, would

for the irreducible Standard Model background.

Single $b$ quark production is enhanced in the Standard Model through the GIM mechanism acting with high mass intermediate top quark states:

$\sigma\left(\nu N \rightarrow \nu b X_{B=0}\right) \propto G_{F}^{4}\left(m_{t}^{4} / M_{W}^{4}\right) \ln \left(m_{t}^{4} / M_{W}^{4}\right)\left(m_{t}^{2} / M_{W}^{2}\right)\left[\left|V_{t d}\right|^{2} D+\left|V_{t s}\right|^{2} S\right] \eta\left(m_{b}^{2} / 2 M E\right)$

(with next-to-leading order QCD corrections available [84]) where $D$ and $S$ represent the down and strange quark contributions from the nucleon, respectively, $\eta\left(m_{b}^{2} / 2 M E\right)$ represents a kinematic threshold suppression from the heavy $b$ quark mass, and the contributions from $D$ and $S$ are likely to be similar. Even so, Eq. 84 predicts the FCNC with a $\nu \nu d b$ vertex to occur only at the level of $10^{-8}$ of the event sample even for $\nu \mathrm{MCs}$ at high enough energies for the $B$ threshold effects to become small. Most likely, therefore, the Standard Model backgrounds will instead come from other processes that have been experimentally misidentified and goal of the analyses will be to search for new physics effects that enhance the FCNC event sample to considerably above the predicted background level.

For single charm quark production, the irreducible Standard Model backgrounds will almost certainly be negligible since, besides the CKM factors, the production amplitude is suppressed in the Standard Model by $m_{b}^{4} m_{t}^{4}$ relative to single $b$ production, although with the caveat that the prediction is more sensitive to long-distance QCD effects that are not currently calculable from first principles. Again, experimentally misidentified events will dominate the backgrounds.

A high performance detector with excellent vertex tagging, lepton identification and reconstruction of event kinematics will be required to cope with large background levels involving both $\mathrm{CC}$ and $\mathrm{NC}$ allowed production of charm or beauty. The allowed CC channels,

$$
\begin{aligned}
& \nu_{\mu} N \rightarrow \mu^{-} c X, \\
& \bar{\nu}_{e} N \rightarrow e^{+} b X,
\end{aligned}
$$

will be most dangerous as $y \rightarrow 1$ and so the very low energy final state muon or electron can escape detection. This background could be suppressed to some 
extent by imposing a cut on the minimum allowed transverse momentum, $p_{t}$, in the event, which can be large for the signal NC events when the neutrino has a large $p_{t}$ but should be zero within the detector resolution for charged current events. Neutral current production of heavy quark pairs

$$
\begin{aligned}
& \nu_{\mu} N \rightarrow \nu_{\mu} c \bar{c} X, \\
& \nu_{\mu} N \rightarrow \nu_{\mu} b \bar{b} X
\end{aligned}
$$

forms the other background when one or other of the heavy quark decays is not picked up by the vertex detector because it occurs too quickly or goes into an unfavorable final state. This background in particular makes setting any stringent limits from FCNC production of charm difficult at $\nu \mathrm{MCs}$, even with an excellent vertexing geometry such as that shown in Fig. 3.

The situation is more promising with $B$ decays, since almost every $B$ decay gives two chances for detection: at the primary $B$ decay vertex and at the decay of the daughter charm hadron. Also, charged current $b$ production may be accompanied by a $\bar{c}$, and this information can be used as well.

Even for FCNC $B$ production at a $\nu \mathrm{MC}$, the only interesting limit may well be for the subset of FCNC $B$ production that occurs off a valence $d$ quark, i.e. involving the FCNC vertices $\nu \nu d b$. This restriction provides two important additional experimental handles: (1) kinematically, almost all of the NC background events will be at relatively low Bjorken $x$ while the PDF for $d$ valence quarks extends to high Bjorken $x$, and $(2) d$ valence quarks will produce $B^{-}$ mesons approximately half the time but never a $B^{+}$, while the $B^{\text {'s forming }}$ the NC background are sign symmetric.

Further discussion on the experimental and theoretical issues for $\nu \nu d b$ FCNC searches at $\nu \mathrm{MCs}$ is given elsewhere[14].

\subsubsection{Generic Four-Fermion Operators}

Neutrino-nucleon processes at low momentum transfer are sensitive to generic four-fermion contact terms produced by the high energy neutral current interactions. Other, flavor changing, couplings are well constrained by the limits on processes like $\pi \rightarrow e \nu$. These four-fermion interactions can be generated in a variety of new physics scenarios. Examples include, but are not limited to, supersymmetric theories with $\mathcal{R}$-parity non-conservation, new vector bosons $Z^{\prime}$ which appear in many superstring-motivated models, models with TeVscale gravity, and quark compositeness models. For instance, the low energy

remnant of a generic high energy electron-quark neutral current interaction 
can be represented by non-zero coupling constants, $\eta$, in the Lagrangian:

$$
\begin{aligned}
\mathcal{L}_{N C}= & \sum_{q}\left[\eta_{L L}^{e q}\left(\overline{e_{L}} \gamma_{\mu} e_{L}\right)\left(\overline{q_{L}} \gamma^{\mu} q_{L}\right)+\eta_{R R}^{e q}\left(\overline{e_{R}} \gamma_{\mu} e_{R}\right)\left(\overline{q_{R}} \gamma^{\mu} q_{R}\right)\right. \\
& \left.+\eta_{L R}^{e q}\left(\overline{e_{L}} \gamma_{\mu} e_{L}\right)\left(\overline{q_{R}} \gamma^{\mu} q_{R}\right)+\eta_{R L}^{e q}\left(\overline{e_{R}} \gamma_{\mu} e_{R}\right)\left(\overline{q_{L}} \gamma^{\mu} q_{L}\right)\right]
\end{aligned}
$$

A similar equation can be written for direct neutrino-quark interactions. One can use $S U(2)$ symmetry to relate $\nu$ and $e$ couplings:

$$
\begin{aligned}
& \eta_{L L}^{\nu u}=\eta_{L L}^{e d}, \\
& \eta_{L L}^{\nu d}=\eta_{L L}^{e u} \\
& \eta_{L R}^{\nu u}=\eta_{L R}^{e u} \\
& \eta_{L R}^{\nu d}=\eta_{L R}^{e d}
\end{aligned}
$$

so that $\nu N$ interactions can be used to constrain the $\eta$ 's in the Lagrangian of Eq. 89.

A particular example of a high-energy model that leads to a low-energy Lagrangian of this type is provided by R-parity-violating $(\mathbb{R})$ SUSY, with the Lagrangian:

$$
\begin{aligned}
\mathcal{L}_{\mathcal{R}} & =\lambda_{i j k}^{\prime}\left(\tilde{e}_{L}^{i} \overline{d_{R}^{k}} u_{L}^{j}+\tilde{u}_{L}^{j} \overline{d_{R}^{k}} e_{L}^{i}+\tilde{d}_{R}^{k *} \overline{e_{L}^{i c}} u_{L}^{j}\right. \\
& \left.-\tilde{\nu}_{L}^{i} \overline{d_{R}^{k}} d_{L}^{j}-\tilde{d}_{L}^{j} \overline{d_{R}^{k}} \nu_{L}^{i}-\tilde{d}_{R}^{k *} \overline{\nu_{L}^{i c}} d_{L}^{j}\right)+ \text { h.c. }
\end{aligned}
$$

At low values of transferred momenta, this Lagrangian can be approximated in terms of local four-fermion interactions:

$$
\begin{aligned}
\mathcal{L}_{e d} & =\frac{\left(\lambda_{1 j 1}^{\prime}\right)^{2}}{m_{\tilde{u}_{L}^{j}}^{2}} \overline{e_{L}} d_{R} \overline{d_{R}} e_{L}+\frac{\left(\lambda_{1 j 1}^{\prime}\right)^{2}}{m_{\tilde{d}_{L}^{j}}^{2}} \overline{\nu_{L}} d_{R} \overline{d_{R}} \nu_{L} \\
& =\left(-\frac{\left(\lambda_{1 j 1}^{\prime}\right)^{2}}{2 m_{\tilde{u}_{L}^{j}}^{2}} \overline{e_{L}} \gamma^{\mu} e_{L}-\frac{\left(\lambda_{1 j 1}^{\prime}\right)^{2}}{2 m_{\tilde{d}_{L}^{j}}^{2}} \overline{\nu_{L}} \gamma^{\mu} \nu_{L}\right) \overline{d_{R}} \gamma_{\mu} d_{R}
\end{aligned}
$$

Assuming that the squarks of first two generations are degenerate and imposing $S U(2)$ symmetry constraints gives

$$
\eta_{L R}^{e d}=-\frac{\left(\lambda_{1 j 1}^{\prime}\right)^{2}}{2 m_{\tilde{u}_{L}^{j}}^{2}}=-\frac{\left(\lambda_{1 j 1}^{\prime}\right)^{2}}{2 m_{\tilde{d}_{L}^{j}}^{2}}=\eta_{L R}^{\nu d}
$$

Indeed, the best constraint on this coupling, $\eta_{L R}^{e d}<0.07_{-0.24}^{+0.24}$ already comes from the analysis of neutrino-nucleon scattering experiments [85]. Data from $\nu \mathrm{MCs}$ should complement new constraints on the new physics contributions that will become available from new Tevatron, LHC and DESY analyses. 


\subsubsection{Heavy Neutral Lepton Mixing}

Another opportunity for $\nu \mathrm{MCs}$ to significantly improve on already existing bounds on new physics from neutrino experiments is provided by the ability to search for the existence of neutral heavy leptons [86]. In several models [87,88], neutral heavy leptons are considered heavy isosinglets that interact and decay by mixing with their lighter neutrino counterparts. The high intensity neutrino beams created by $\nu \mathrm{MCs}$ provide an ideal setting to search for neutral heavy leptons with a mass below $100 \mathrm{MeV}$.

It is postulated that neutral heavy leptons, $L_{0}$, could be produced from muon decays when one of the neutrinos from the decay mixes with its heavy, isosinglet partner. The expression for the number of neutral heavy leptons produced in a muon beam is:

$$
\begin{aligned}
N_{L_{0}} & =N_{\nu} \times B r\left(\frac{\mu \rightarrow L_{0} \nu e}{\mu \rightarrow \nu \nu e}\right) \times \epsilon \times e^{-L / \gamma c \tau} \\
& \times B r\left(\frac{L_{0} \rightarrow \text { detectable }}{L_{0} \rightarrow \text { total }}\right) \times\left(1-e^{-\delta l / \gamma c \tau}\right) .
\end{aligned}
$$

Here $N_{\nu}$ is the number of neutrinos produced from muon decays, $\operatorname{Br}(\mu \rightarrow$ $\left.L_{0} \nu e / \mu \rightarrow \mu \nu e\right)$ is the branching ratio of muons decaying into neutral heavy leptons versus ordinary muon decays, $L$ is the distance from the beam-line to the detector, $\delta l$ is the length of the detector, $\epsilon$ is the combined detector efficiency and geometric efficiency, $\tau$ is the $L_{0}$ lifetime, and $\operatorname{Br}\left(L_{0} \rightarrow\right.$ detectable $/ L_{0} \rightarrow$ total) is the branching ratio for the neutral heavy lepton decaying via a detectable channel (mainly, $L_{0} \rightarrow \nu e e$ ).

Note here that the muon has two possible ways of producing $L_{0}$ 's:

$$
\begin{aligned}
& \mu^{-} \rightarrow L_{0}+\bar{\nu}_{e}+e^{-} \\
& \mu^{-} \rightarrow \nu_{\mu}+\bar{L}_{0}+e^{-} .
\end{aligned}
$$

The branching ratio for each of these reactions is given by:

$$
\begin{aligned}
& B r\left(\mu \rightarrow L_{0} \bar{\nu}_{e} e / \mu \rightarrow \nu_{\mu} \nu_{e} e\right)=\left|U_{\mu L}\right|^{2}\left(1-8 x_{m}^{2}+8 x_{m}^{6}-x_{m}^{8}+12 x_{m}^{4} \ln x_{m}^{2}\right) \\
& \operatorname{Br}\left(\mu \rightarrow \nu_{\mu} \bar{L}_{0} e / \mu \rightarrow \nu_{\mu} \nu_{e} e\right)=\left|U_{e L}\right|^{2}\left(1-8 x_{m}^{2}+8 x_{m}^{6}-x_{m}^{8}+12 x_{m}^{4} \ln x_{m}^{2}\right) .
\end{aligned}
$$

Here $x_{m}$ is defined as $m_{L_{0}} / m_{\mu}$ and $\left|U_{(\mu, e) L}\right|^{2}$ is defined as the coupling constant between the specific type of neutrino and the neutral heavy lepton.

Once produced, a neutral heavy lepton of such low mass will either decay via 
three neutrinos $\left(L_{0} \rightarrow \nu \nu \nu\right)$, or through the channels:

$$
\begin{aligned}
& L_{0} \rightarrow \nu_{\mu}+e^{+}+e^{-} \\
& L_{0} \rightarrow \nu_{e}+e^{+}+e^{-} .
\end{aligned}
$$

The first decay mode involves only the neutral current, whereas the second contains a mixture of both neutral and charged current interactions. The branching ratios for decay processes (99) and (100) have been calculated[89]. Note that the number of $L_{0}$ 's detectable at the $\nu \mathrm{MC}$ depends roughly on $U^{4}$.

Using the above model with some additional assumptions, one can estimate the number of neutral heavy leptons produced at the $\nu \mathrm{MC}$ that then decay to two electrons and a neutrino and are detected in an experiment. The plots in Fig. 15 show limits for the coupling constants at a $\nu \mathrm{MC}$ as a function of the $L_{0}$ mass and for a number of different muon energies and detector distances. All the plots assume a pure, unpolarized muon beam containing $10^{20}$ muons/year with straight sections such that 25 percent of the muons will decay to neutrinos pointing towards the detector. The detector parameters are based on the detector for the $\mathrm{NuTeV} L_{0}$ searches[90-92]: 3 meters in diameter and 30 meters in length and with enough resolution to detect the $e^{+} e^{-}$vertex from the $L_{0}$ decay. Finally, it is assumed that backgrounds are negligible.

It is seen from Fig. 15 that one achieves the best limits from using relatively low energy muon beams. This is a significant improvement over previous neutral heavy lepton searches, where limits fail to reach below $6.0 \times 10^{-6}$ in the low mass region.

The $\nu$ MC may prove to be an ideal location to continue the search for neutral heavy leptons. The high intensity neutrino beam allows for a neutral heavy lepton search to be sensitive to coupling constants in the low mass region. In addition, such a neutral heavy lepton program could easily interface with an already existing detector utilizing the neutrino beam. It is also clear that a neutral heavy lepton search would receive the most benefit at lower muon energies, and thus would yield best results at lower energy $\nu \mathrm{MCs}$.

\subsection{Studies of Low Energy QCD}

\subsection{1 $\bar{\nu}_{e} e^{-}$Annihilation}

Section 4.2 showed that neutrino-electron elastic and quasi-elastic scattering can be used to extract the electroweak parameter $\sin ^{2} \theta_{W}$ from purely leptonic interactions. This section instead discusses the neutrino-electron annihilation 

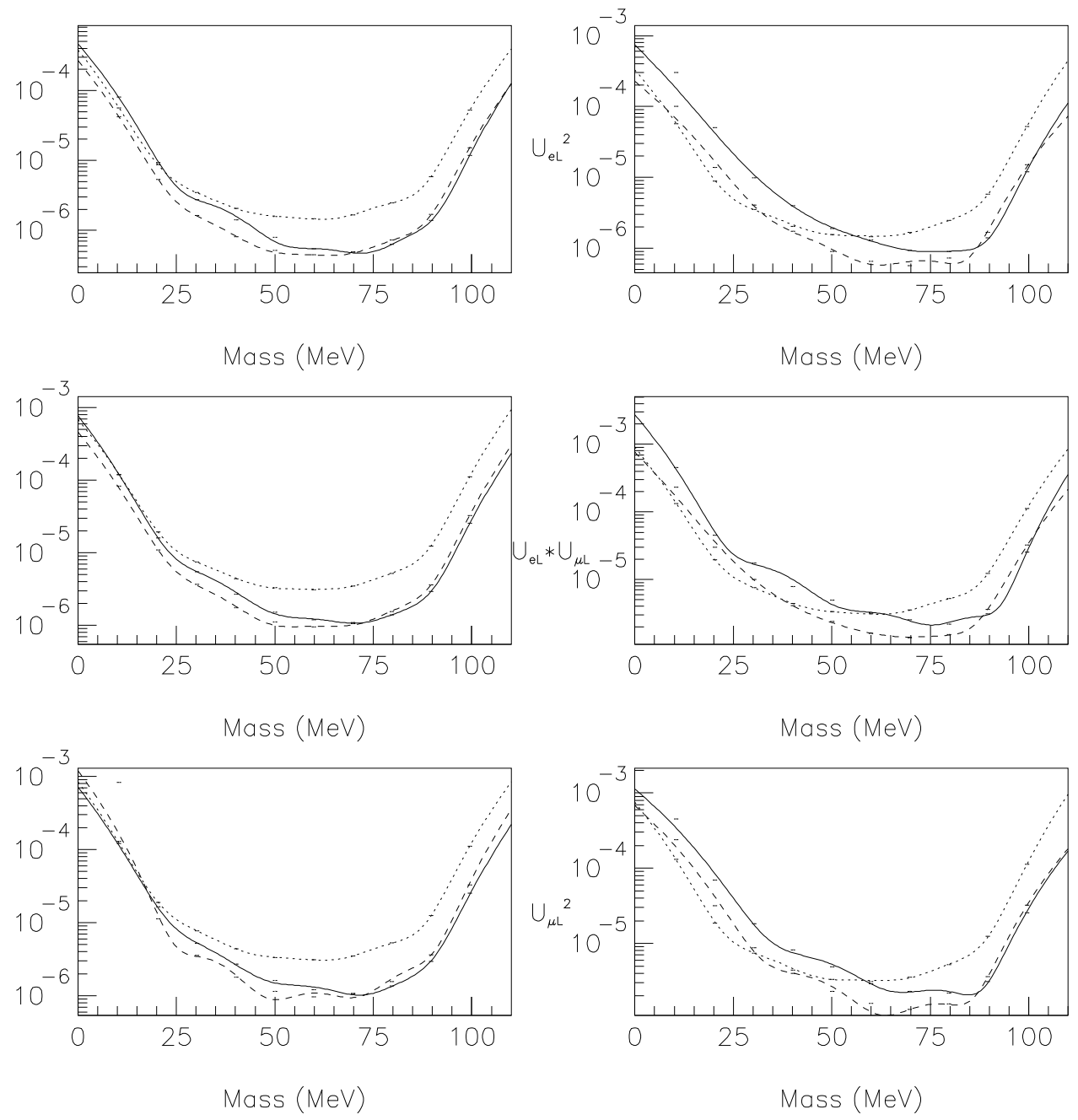

Fig. 15. Plots for limits on the (from top to bottom) coupling values $\left|U_{e L}\right|^{2},\left|U_{\mu L} U_{e L}\right|$ and $\left|U_{\mu L}\right|^{2}$ as a function of $L_{0}$ mass for one year of running under the assumptions given in the text. The solid, dashed, and dotted lines represent $\nu \mathrm{MC}$ energies of 10 , 20 , and $250 \mathrm{GeV}$ respectively. The plots on the left show limits for $L=12 \mathrm{~m}$, and the plots on the right show limits for $L=1 \mathrm{~km}$.

processes of Eq. 67,

$$
\bar{\nu}_{e} e^{-} \rightarrow \bar{\nu}_{\mu} \mu^{-}, \bar{\nu}_{\tau} \tau^{-}, \bar{u} d \ldots
$$

Electron antineutrino-electron annihilation is expected to show considerable complexity once the center of mass energy exceeds the threshold for annihilation into hadronic final states. The process can be compared to $e^{+} e^{-} \rightarrow$ hadrons but with the $\rho, \omega, \phi$ and other low-lying vector resonances replaced by the $\pi^{-}, \rho^{-}$, and $a_{1}^{-}$. The axial component of the weak current produces coupling to axial vector resonances and, at low energy, to the pion. These latter couplings are absent in electron-positron annihilation and thus the weak annihilation of the electron offers a novel complement to electron-positron physics. Direct 
Table 6

\begin{tabular}{|c|c|}
\hline final state & $\mathbf{E}_{\nu}(\mathbf{G e V})$ \\
\hline $\bar{\nu}_{\mu} \mu^{-}$ & 11 \\
\hline$\gamma \pi^{-}$ & 19 \\
\hline$\pi^{0} \pi^{-}$ & 76 \\
\hline$(\pi \pi \pi)^{-}$ & 172 \\
\hline$\gamma K^{-}$ & 240 \\
\hline$(K \pi)^{-}$ & 358 \\
\hline$(K \pi \pi)^{-}$ & 446 \\
\hline
\end{tabular}

Threshold neutrino energies for various hadronic final states.

measurements of $\bar{\nu}_{e} e^{-}$annihilation would complement the detailed studies of hadronic tau decays that currently provide some of the most powerful tests of QCD at low momentum transfer.

These measurements bridge two asymptotic limits of QCD: the perturbative regime operative at high $Q^{2}$ and the chiral limit $\left(m_{u}=m_{d}=m_{s}=0\right)$ that is approached at low $\sqrt{s}$, and would provide some important information about the QCD spectral functions used in reducing the theoretical hadronic uncertainties in studies of $C P$-violation in kaon decays, particularly in the interpretation of $\epsilon^{\prime} / \epsilon$ measurements [77,78]. Unfortunately, since the $\tau^{-} \nu_{\tau}$ threshold occurs at $E_{\nu}=3.1 \mathrm{TeV}$, there is a long way to go in neutrino energy in order to completely overlap the $\sqrt{s}$ region probed by tau decays.

Table 6 summarizes neutrino energy thresholds for $s$-channel final states. The thresholds for all channels are quite high. Nevertheless, one can see that a $50 \mathrm{GeV}$ muon storage ring could provide access to the lowest lying final states and explore the interesting region near $\sqrt{s}=m_{\pi}$ to provide a clean test of PCAC, and a $250 \mathrm{GeV}$ muon beam would extend the reach up to the threshold for kaon production.

\subsection{2 $W^{*} / Z^{*}$-photon Scattering}

Just as intense neutrino beams open up the possibility of directly exploring weak annihilation of leptons in analogy to $e^{+} e^{-}$annihilation, so too does one acquire access to the analog of two-photon physics: the scattering of virtual $W$ or $Z$ beams from quasi-real photons in the Coulomb field of the nucleus. The general reaction is of the form

$$
\nu_{\ell} A \rightarrow f_{\ell} F \bar{f} A,
$$


where $F, \bar{f}$ are fermions, and $f_{\ell}=\nu_{\ell}$ or $\ell^{-}$. For $F \bar{f}=\nu_{\ell} \ell^{+}$both CC and NC diagrams contribute. Otherwise $f_{\ell}=\ell^{-}$proceeds through $W \gamma$ scattering and $f_{\ell}=\nu_{\ell}$ through $Z \gamma$ scattering.

The creation of lepton pairs in the Coulomb field of the nucleus is sensitive to $W-Z$ interference and provides a direct test of the Standard Model. Previous experimental observations are ambiguous[79,80,53]. The purely coherent part of the cross section for $\nu_{\ell_{1}} A \rightarrow \ell_{1}^{-} \nu_{\ell_{2}} \ell_{2}^{+}$at asymptotically large energies is $[81,82]$

$$
\begin{aligned}
\sigma_{\ell_{1} \ell_{2}}(E \rightarrow \infty) & =\frac{4 Z^{2} \alpha^{2} G_{F}^{2}}{9 \pi^{3}} E K_{\ell_{1} \ell_{2}}(E, A)(1-\chi) \\
K_{\ell_{1} \ell_{2}}(E, A) & =\frac{5 \pi \beta(A)}{32}\left(\log \frac{2 E \beta(A)}{\rho_{\ell_{1} \ell_{2}}}+\frac{1}{3} \log \frac{2 E \beta(A)}{\rho_{\ell_{1} \ell_{2}}}-R_{\ell_{1} \ell_{2}}\right) .
\end{aligned}
$$

$K_{\ell_{1} \ell_{2}}(E, A)$ is a reaction and nucleus-dependent form factor with $\beta(A) \simeq$ $A^{-1 / 3} \mathrm{fm}^{-1}=6 A^{-1 / 3} \mathrm{GeV}$ set by the nuclear size, and $\rho_{\ell_{1} \ell_{2}}, \rho_{\ell_{1} \ell_{2}}, R_{\ell_{1} \ell_{2}}$ are simple functions of either $\beta(A)$ or final state fermion masses. The factor $Z^{2} \alpha^{2}$ reflects the coherent electromagnetic nature of the process; and the scale of the cross section is set by $G_{F}^{2} \beta_{I} E$. Since the effective center-of-mass energy is $\sqrt{s} \simeq \sqrt{2 \beta_{I} E}$ and $\beta_{I} \gg m_{e}$, all possible leptonic final states are accessible (including those with $\tau^{ \pm}$) to a neutrino beam derived from a $250 \mathrm{GeV}$ muon beam.

The factor $\chi$ incorporates effects of neutral currents, including interference in the $\ell^{+} \ell^{-}$final states. A nice electroweak test is to measure $\chi$ through the ratios

$$
\frac{\sigma_{\mu \mu}\left(\nu_{\mu} A \rightarrow \mu^{-} \mu^{+} \nu_{\mu} A\right)}{\sigma_{\mu e}\left(\nu_{\mu} A \rightarrow \mu^{-} e^{+} \nu_{e} A\right)}=(1-\chi) \frac{K_{\mu \mu}(E, A)}{K_{\mu e}(E, A)}
$$

where $K_{\mu \mu}(E, A) / K_{\mu e}(E, A)$ will depend only weakly on nuclear form factor and energy.

Hadronic resonances are also possible. We expect similar states to those produced in $\gamma \gamma$ collisions, namely $0^{-+}, 0^{++}, 2^{++}, \ldots$ with $I=0$ and $I=1$. Some Cabibbo-allowed examples include

$$
\begin{aligned}
& \nu_{e} A \rightarrow e^{-} \pi^{+} A \\
& \nu_{e} A \rightarrow \nu_{e} \pi^{0} A, \nu_{e} \eta A, \nu_{e} \eta^{\prime} A \\
& \nu_{e} A \rightarrow e^{-} a_{1}^{+} A \\
& \nu_{e} A \rightarrow \nu_{e} f_{0} A, \nu_{e} a_{1}^{0} A \\
& \nu_{e} A \rightarrow e^{-} D_{S}^{+} A .
\end{aligned}
$$

Unfortunately, some of these hadronic resonances do not provide a unique experimental signature. Single pion production, for example, can occur through 
the diffractive process $\nu_{e} A \rightarrow e^{-} \pi^{+} A$ mediated by a collision of the pion component of the virtual $W$ boson with a pomeron from the nucleus or nucleon.

Experimental backgrounds that have to be controlled for these studies include coherent meson production mediated by $W / Z$-pomeron scattering. Especially tricky are the diffractive $D_{s}^{*} / D_{s}$ channels where the $D_{s}$ undergoes two-body $\tau$ decay,

$$
\nu_{\ell} A \rightarrow \ell^{-} D_{s}^{+*} / D_{s}^{+} A \rightarrow \ell^{-}(\gamma) \tau^{+} \nu_{\tau} \rightarrow \ell^{-}(\gamma) \ell^{\prime+} \nu_{\tau} \bar{\nu}_{\tau} \nu_{\ell^{\prime}}
$$

Also of concern are backgrounds from inclusive charged current charm production, $\nu_{\mu} N \rightarrow \mu^{-} c X$, where the charm quark fragments into a charmed hadron which takes nearly all of the event's hadronic energy and then decays semi-leptonically into a final state where leptons carry nearly all the energy.

\subsection{Conclusions on Rare and Exotic Processes at $\nu M C s$}

Rare processes that could be studied at a $\nu \mathrm{MC}$ would probe, both directly and indirectly, an energy range from fractions of a $\mathrm{GeV}$ to above the $\mathrm{TeV}$ scale. This would provide important information complementary to the existing results in some areas (e.g., in low energy QCD studies, FCNC, contact interactions) and could substantially improve current bounds on the parameters of some new physics models (e.g. heavy neutral lepton searches).

\section{Charm Decay Physics}

\subsection{Introduction}

A $\nu \mathrm{MC}$ will constitute a rather impressive charm factory. Figures 4 and 5 of Sec. 1 show that one can expect between $2 \times 10^{8}$ and $2 \times 10^{9}$ well-reconstructed charm events in a total event sample of $10^{10}$ events, depending on the $\nu \mathrm{MC}$ energy. Several species of charmed hadrons should be produced, with measured [93] relative production fractions for the more common charmed hadrons of:

$$
D^{0}: D^{+}: D_{S}^{+}: \Lambda_{C}^{+}=0.60: 0.20: 0.10: 0.10
$$

Also, the $\Sigma_{C}^{++}$and $\Sigma_{C}^{+}$are expected[94] to have comparable production cross sections to $\Lambda_{C}^{+}$. The charmed-strange baryons $\Xi_{C}^{+}$and $\Xi_{C}^{0}$ should be produced at levels down by a factor of a few and $\Omega_{c}$ should be still less common. 
The ratios of Eq. 105 are relatively independent of the neutrino energy for energies above $10 \mathrm{GeV}$ and are for production from neutrinos; the corresponding antiparticles containing anti-charm will be produced from antineutrinos in similar ratios, although with differences in the absolute cross sections and kinematic distributions. The large asymmetry between $D^{0}$ and $D^{+}$production is due to the prevalence of $D^{*}$ production with its preference for decays into $D^{0}$.

As well as providing good all-around event reconstruction, $\nu \mathrm{MCs}$ will have two other distinct and important experimental advantages over all other types of charm facilities. Firstly, reconstruction of the charm decay vertex should be superior to that at any collider experiment, particularly for the reconstruction of the challenging 1-prong charm decays, as was illustrated by Fig. 3. Secondly, a uniquely pure and efficient tag of whether the production flavor is charm or anti-charm is provided by the $100 \%$ correlated sign of the primary lepton from the interaction:

$$
\begin{aligned}
& \nu_{\ell} q \rightarrow \ell^{-} c \\
& \bar{\nu}_{\ell} \bar{q} \rightarrow \ell^{+} \bar{c} .
\end{aligned}
$$

This section discusses several areas for charm decay physics at $\nu \mathrm{MCs}$ where these experimental capabilities should be important. The theoretical interest of each measurement will be discussed, and brief summaries of the expected experimental techniques and sensitivities at $\nu \mathrm{MCs}$ will be included. Expected relative strengths and weaknesses of $\nu \mathrm{MCs}$ compared to other future charm facilities will also be touched on. However, detailed numerical predictions for measurement precisions await more extensive feasibility studies than have been performed for this report.

There are also possibilities for $B$ decay physics using the neutrinos from multiTeV muon colliders[14,9], where $b \bar{b}$ production in neutral current interactions should be at the level of $10^{-3}$ of the total cross section. Associated production of $b \bar{c}$ and $c \bar{b}$ can also be studied, however the relevant production cross-sections are suppressed by approximately two orders of magnitude compared to the $b \bar{b}$ production cross section.

\subsection{Theoretical Motivation for Charm Physics}

It is clear that, from the point of view of Standard Model electroweak physics, charm decays represent a decidedly dull affair. First, the relevant CKM parameters are reasonably well known, for the smallness of $\left|V_{c b}\right|$ and $\left|V_{u b}\right|$ constrains $V_{c s}$ and $V_{c d}$ very tightly through three-family unitarity (see Sec. 3). Second, $D^{0}-\bar{D}^{0}$ oscillations proceed slowly. Third, $C P$ asymmetries are small due to 
the fact that both decaying and final state particles contain quarks of only the first two generations. Finally, rare charm decay rates are tiny and, again, are dominated by long-distance effects.

These apparent vices can, however, be turned into virtues. Since the weak dynamics apparently hold no secrets, one can employ charm decays as a laboratory to study QCD in the interface of perturbative and non-perturbative dynamics. Also, precisely because the Standard Model promises us no drama in charm decays, one can conduct searches for $D^{0}-\bar{D}^{0}$ oscillations, $C P$ violation and rare charm decays as probes for new physics with almost no background from the Standard Model.

\subsection{Probing Strong Interactions through Charm Decays}

Improved measurements of charm decays are needed for phenomenological and theoretical reasons even in the absence of new physics, for the following reasons:

- to improve the data base needed for analyzing $B$ decays one needs more precise measurements of the absolute branching ratios of charm hadrons;

- measurements of the leptonic decay rates $D_{(s)} \rightarrow \ell \nu$ are required for determining the meson decay constants; these decay constants give us quantitative insight into the dynamics of heavy-light bound state systems and can be used for tuning the lattice QCD methods and a more reliable evaluation of $B^{0}-\bar{B}^{0}$ oscillations;

- more precise studies of inclusive semileptonic $D, D_{s}, \Lambda_{c}$, etc. decays would provide us with valuable novel insights into the inner workings of QCD and at the same time sharpen our tools for a quantitative treatment of $B$ decays.

\subsubsection{Absolute Charm Branching Ratios}

As the discussion about the charm content in the final state of $B$ decays illustrates, a significant bottleneck in the detailed analysis of beauty decays of the $b \rightarrow c$ type is currently caused by the uncertainties in the absolute branching ratios of charm hadron decays to specified final states, in particular of $D_{s}, \Lambda_{c}$ and $\Xi_{c}$. A $\nu \mathrm{MC}$ should be well suited to obtaining these branching ratios, as we now discuss. This information will be useful even if it is obtained only after the next generation of $B$ experiments have accumulated their samples.

As what is typically the less difficult part of the measurements, the expected excellent particle identification and event reconstruction at $\nu \mathrm{MCs}$ should give good capabilities for determining relative branching ratios for each hadron. The more difficult task of obtaining the production normalization factors to 
convert these to absolute branching ratios should then be achieved by fitting the experimental decay length distributions in a procedure that was studied for the COSMOS (E803) neutrino experiment at Fermilab.

The COSMOS technique[95] envisions fitting normalization factors to the several known decay exponentials - one for each charmed hadron species - in the observed neutral and charged distributions for the variable $x=d / p$, with $d$ the charmed hadron distance to the decay vertex and $p$ its reconstructed momentum. It is helpful that the exponential decay constants in this variable are well separated for both the charged and neutral hadron distributions:

$$
\begin{aligned}
x\left(D^{+}\right) & =170 \mu \mathrm{m} /(\mathrm{GeV} / \mathrm{c}) \\
x\left(D_{S}^{+}\right) & =71 \mu \mathrm{m} /(\mathrm{GeV} / \mathrm{c}) \\
x\left(\Xi_{C}^{+}\right) & =43 \mu \mathrm{m} /(\mathrm{GeV} / \mathrm{c}) \\
x\left(\Lambda_{C}^{+}\right) & =27 \mu \mathrm{m} /(\mathrm{GeV} / \mathrm{c}),
\end{aligned}
$$

and

$$
\begin{aligned}
& x\left(D^{0}\right)=67 \mu \mathrm{m} /(\mathrm{GeV} / \mathrm{c}) \\
& x\left(\Xi_{C}^{0}\right)=12 \mu \mathrm{m} /(\mathrm{GeV} / \mathrm{c}) \\
& x\left(\Omega_{C}^{0}\right)=7 \mu \mathrm{m} /(\mathrm{GeV} / \mathrm{c})
\end{aligned}
$$

Auxiliary information for the fit will be available from particle identification in the detector. In particular, the presence of a proton in the final state will reliably indicate the decay of a baryon rather than a meson.

To test the method for the COSMOS environment, exponential fits were performed [95] for simulated decay length distributions from approximately 14000 reconstructed $D^{+}, D_{S}^{+}$and $\Lambda_{C}^{+}$charm decays. The fitted statistical uncertainties for the three species were $3.4 \%, 12 \%$ and $5.4 \%$, respectively. These simulations show that statistical uncertainties would be negligible for such a fit at a $\nu \mathrm{MC}$, which would have several orders of magnitude more events. The uncertainties in the charm production rates would instead be dominated by uncertainties in modeling the level of vertexing inefficiencies. Hopefully, these uncertainties could also be made small due to the favorable vertexing geometry shown in Fig. 3 and to the considerable potential for using the data itself to estimate the inefficiencies.

Another area where $\nu \mathrm{MCs}$ can be expected to make significant or even unique contributions is in the analysis of final states that contain more than one neutral hadron, e.g.,

$$
D^{0} \rightarrow \pi^{+} \pi^{-} \pi^{0} \pi^{0} ; D^{+} \rightarrow \pi^{+} \pi^{0} \pi^{0} ; D_{s}^{+} \rightarrow \pi^{+} \pi^{0} \eta \text { etc. } .
$$


Even all neutral final states like

$$
D^{0} \rightarrow 2 \pi^{0}, 3 \pi^{0}
$$

might become observable.

Such neutral-rich channels are rather elusive for the usual $e^{+} e^{-}$annihilation and photoproduction experiments. A $\nu \mathrm{MC}$ could access these modes through the expected sample of $10^{6-7} \mathrm{NC}$-produced $c \bar{c}$ events. Vertex tagging one of the charmed hadrons would allow a search for such decay modes in the other. Filling in these 'white spots' in the map of charm decays would close or at least narrow the gap between exclusive and inclusive decays and thus can provide us with important lessons on how quark-hadron duality is realized in subclasses of total decays. For example: a quark based description leads to the prediction that the (Cabibbo suppressed) inclusive rates driven by $c \rightarrow s \bar{s} u$ and $c \rightarrow d \bar{d} u$ should practically coincide since $m_{d}, m_{s} \ll m_{c}$. Yet exclusive channels like $D^{0} \rightarrow K^{+} K^{-}$and $D^{0} \rightarrow \pi^{+} \pi^{-}$do not at all follow this expectation! Duality suggests that a (near) equality will emerge for $\Gamma\left(D \rightarrow K \bar{K}+\pi^{\prime} s\right)$ vs. $\Gamma(D \rightarrow$ $\left.\pi^{\prime} s\right)$. Testing this expectation requires the measurement of final states with neutrals.

Experimental studies of multi-body decays with more than one neutral meson in the final state (in particular, Dalitz plot analyses) also allow us to have different handles on the studies of direct $C P$-violation in D-decays in and beyond the Standard Model [96] as well as on the dynamics of hadronic resonances governing these transitions (see, e.g. the E791 analysis $[97,98]$ ).

\subsection{2 $D_{s}, D^{+} \rightarrow \mu^{+} \nu, \tau^{+} \nu$}

The primary goal behind measuring leptonic decays, $D_{s} \rightarrow \ell^{+} \nu$, or the Cabibbo suppressed versions, $D^{+} \rightarrow \ell^{+} \nu$, with $\ell=\mu, \tau$, is the desire to extract the decay constants $f_{D_{s}}$ and $f_{D}$. These quantities are important probes of heavy meson wave functions. In addition, these decay constants have been extracted from Monte Carlo simulations of QCD on the lattice with estimated uncertainties of about 20 percent on their absolute values and about 10 percent on their ratio. Improvements are expected for future lattice calculations. For the proper evaluation of these calculations, one wants to calibrate them against experimental results of similar accuracy.

Currently, the branching ratios for $D_{s} \rightarrow \ell \nu$ transitions have been measured by the CLEO collaboration with large uncertainties, $\operatorname{Br}\left(D_{s} \rightarrow \mu \nu\right)=4.0_{-2.0}^{+2.2} \times$ $10^{-3}$ and $\operatorname{Br}\left(D_{s} \rightarrow \tau \nu\right)=(7 \pm 4) \times 10^{-2}$. No measurement is currently available for other $D$ mesons although there is an upper bound: $\operatorname{Br}\left(D^{+} \rightarrow \mu \nu\right)<$ $7.2 \times 10^{-2}$. This can be explained by the $\lambda=0.2$ CKM suppression factor for the $D^{+}$leptonic decays relative to those of the $D_{s}$. 
Once the absolute values of $f_{D}$ or $f_{D_{s}}$ are known experimentally with about $\%$ accuracy or better then one will be able to feel more confident about extrapolating to the decay constants in the $B$ system, $f_{B}$ and $f_{B_{s}}$, which are crucial quantities for a quantitative understanding of $B^{0}-\bar{B}^{0}$ oscillations and the extraction of $V_{t d}$ from them.

Observing and measuring these transitions has always represented a highly nontrivial experimental challenge (and much more so for $D^{+} \rightarrow \ell^{+} \nu$ ), so the potentially exceptional performance for observing 1-prong $\mathrm{D}$ decays at $\nu \mathrm{MC}$ 's could allow them to make a significant contribution here even down the line. As a secondary goal one might even perform a detailed comparison of the rates for $D \rightarrow \mu \nu$ and $D \rightarrow \tau \nu$ as a probe for new physics in the form of a non-minimal Higgs sector, for charged Higgs exchanges would affect the latter much more than the former.

\subsubsection{Inclusive Charm Hadron Decays}

Heavy quark expansions (HQE) allow the treatment of inclusive heavy flavor decays, including their non-perturbative aspects [99-103]. In addition to total decay widths, other central quantities are inclusive semileptonic branching ratios and decay spectra for the different meson and baryon species. These techniques provide the basis for some of the most reliable methods for extracting $\left|V_{c b}\right|$ and $\left|V_{u b}\right|$ in $B$ decays. Obviously one wants to cross check these methods in a system where the CKM parameters are known, namely the charm system, by testing how precisely $\left|V_{c s}\right|$ and $\left|V_{c d}\right|$ can be extracted from semileptonic charm decays. In addition one can extract the size of the matrix elements of four-fermion operators that are of direct relevance in beauty decays and at the same time provide important calibration points for lattice simulations of QCD.

No data of sufficient detail are available. The $B$ factories (CLEO, BaBar and Belle) will significantly improve the situation, but might not achieve the desired experimental accuracy. Furthermore, it turns out that comparing neutrino with charged lepton spectra in semileptonic decays provides us with particularly probing insights.

One has to keep the following in mind. Since the expansion parameter is $\mu_{\text {had }} / m_{c}$ with $\mu_{\text {had }} \sim 0.7-1 \mathrm{GeV}$, one has to allow for uncalculated higher order contributions to modify the results significantly in charm decays. To have a handle on this complication, one needs to be able to perform detailed comparisons of the lepton spectra separately in $D^{0}, D^{+}, D_{s}$ and $\Lambda_{c}$ decays, which should be possible at $\nu \mathrm{MC}$ 's.

FOCUS and SELEX data will presumably yield precise lifetimes for $\Xi_{c}^{0,+}$ baryons, but quite possibly not for the $\Omega_{c}$. The latter is presumably the short- 
est lived hadron in the single charm sector, with $\tau\left(\Omega_{c}\right)<10^{-13}$ sec; due to its different spin structure its lifetime is affected by different matrix elements than for the other baryons. It is also quite unclear whether next generation experiments like LHC-B and BTeV can measure such a short lifetime with good accuracy. A $\nu \mathrm{MC}$ thus could make a relevant measurement that would serve as an a posteriori calibration of some theoretical tools. Furthermore, a whole new spectroscopy could be entered into, namely that of baryons carrying two units of charm: $[c c q]$.

In principle, radiative inclusive (and exclusive) decays can also be studied. The predicted branching ratio for the short-distance contribution is tiny, $\operatorname{Br}(c \rightarrow$ $u \gamma)=(4.2-7.9) \times 10^{-12}[104]$, although two-loop QCD corrections could bring it up to $5 \times 10^{-8}$ [105]. This could have made it a sensitive probe of new physics as these processes occur in the Standard Model only at one loop. Unfortunately, the problem is that the long-distance effects can actually completely dominate this decay, enhancing it up to $\sim 10^{-5}$, and these enhancements cannot be estimated model-independently.

\subsection{Searches for New Physics in Charm Decays}

\subsection{1 $D^{0}-\bar{D}^{0}$ Oscillations}

The phenomenon of meson-antimeson mixing has been studied both experimentally and theoretically for a long time as it provides an extremely sensitive test of the Standard Model as well as its various possible extensions. This is especially true for $D^{0}-\bar{D}^{0}$ mixing, as was already indicated.

To study such oscillations one must tag separately the flavor of the produced meson and of the decaying meson. The charge of the primary lepton from a $\mathrm{CC}$ interaction uniquely tags the production sign of the charm quark method at an $\nu \mathrm{MC}$. This should easily be the cleanest and most efficient tag. It can be checked by a more conventional alternative method involving production of the charged $D^{*}$ mesons and studies of the decay chain $D^{* \pm} \rightarrow D^{0}\left(\bar{D}^{0}\right) \pi^{ \pm}$ $[106,107]$, where anti-correlation studies of the charge of $\pi$ and decay products of $D$ would reveal whether mixing took place.

For charmed mesons with tagged production flavor, $D^{0}-\bar{D}^{0}$ oscillations are most cleanly probed through 'wrong-sign' semileptonic decays with the branching ratios:

$$
r_{D}=\frac{\Gamma\left(D^{0} \rightarrow \ell^{-} X\right)}{\Gamma\left(D^{0} \rightarrow \ell^{+} X\right)} \simeq \frac{1}{2}\left(x_{D}^{2}+y_{D}^{2}\right), x_{D}=\frac{\Delta m_{D}}{\Gamma_{D}}, y_{D}=\frac{\Delta \Gamma_{D}}{2 \Gamma_{D}}
$$

for $\left.\Delta m_{D}=m_{(} D^{0}\right)-m_{\bar{D}^{0}}, \Gamma_{D}$ the $D$ width and $\Delta \Gamma_{D}$ the difference in the $D^{0}$ 
and $\bar{D}^{0}$ mass widths.

In principle, one can determine the flavor of the final state through charged kaons; mis-tags that happen due to doubly Cabibbo suppressed decays can be eliminated using a time-dependent analysis, as discussed below.

The most recent experimental limits, which are from fixed target experiments at FNAL and from CLEO at CESR and combine tagging through 'wrong' sign leptons and kaons, read:

$$
\begin{aligned}
r_{D} & \leq 5 \times 10^{-4}, 95 \% \text { C.L.; CLEO }[108] \\
-0.04 & \leq y_{D} \leq 0.06,90 \% \text { C.L.; } \operatorname{E791}[109] \\
-0.058 & \leq y_{D}^{\prime} \leq 0.01,95 \% \text { C.L.; } \text { CLEO }[108]
\end{aligned}
$$

where

$$
y_{D}^{\prime} \equiv y_{D} \cos \delta_{K \pi}-x_{D} \sin \delta_{K \pi}
$$

with $\delta_{K \pi}$ denoting the strong phase shift between $D^{0} \rightarrow K^{+} \pi^{-}$and $\bar{D}^{0} \rightarrow$ $K^{+} \pi^{-}$(see [110] for the recent analysis), and

$$
y_{C P}=0.0342 \pm 0.0139 \pm 0.0074 ; \quad \text { FOCUS[111] }
$$

where $y_{C P}=y_{D}$ in the Standard Model. Since possible new physics effects or hadronic uncertainties will affect these experiments differently, a careful analysis to extract the true values of $\Delta m_{D}$ and $\Delta \Gamma_{D}$ from the data should be performed [112]. The $B$ factories at Cornell, SLAC and KEK will refine the search for $D^{0}-\bar{D}^{0}$ oscillations to an expected sensitivity of $r_{D} \sim$ few $\times 10^{-4}$ [113].

While the Standard Model undoubtedly predicts slow $D^{0}-\bar{D}^{0}$ oscillations $x_{D}, y_{D} \ll 1-$ there is considerable uncertainty in the numerical predictions. A conservative Standard Model bound is given by [114,115]

$$
\left.r_{D}\right|_{S M}<10^{-4} \simeq y_{D},\left.x_{D}\right|_{S M} \leq 10^{-2}
$$

Bolder predictions have been made that $x_{D}$ and $y_{D}$ cannot exceed $10^{-3}[114,115]$ and therefore $r_{D} \leq 10^{-6}$ within the Standard Model. On the other hand, new physics could enhance $x_{D}$ up to, and actually even above, the present bound,

$$
\left.x_{D}\right|_{N P} \sim 0.1
$$

without violating any other limit and while leaving $y_{D}$ unaffected. Examples of such new physics processes include various supersymmetric models [116] (including SUSY models with quark-squark alignment that actually require 
$\Delta m_{D}$ close to the current experimental bound) [117], models with singlet up quarks [118], various leptoquark models [119], and multiscalar models with [120], and without [121], natural flavor conservation. Any experimental effort to lower the current limit on $\Delta m_{D}$ is essential in determining the available parameter space for many possible extensions of the Standard Model!

The cleanest way to probe for $D^{0}-\bar{D}^{0}$ oscillations is to analyze the time evolution of transitions into 'wrong-sign' leptons:

$$
\Gamma\left(D^{0}(t) \rightarrow \ell^{+} X\right) \propto e^{-t / \tau_{D}} x_{D}^{2}\left(\frac{t}{\tau_{D}}\right)^{2}
$$

Here we have invoked the $\Delta Q=-\Delta C$ rule of the Standard Model which makes oscillations the only source for wrong-sign leptons.

Since one is embarking on a search for new physics, one should generalize equation 119 to allow for a violation of the $\Delta Q=-\Delta C$ rule, giving:

$$
\begin{gathered}
\Gamma\left(D^{0}(t) \rightarrow \ell^{+} X\right) \propto e^{-t / \tau_{D}} \times \\
{\left[\left(1+\frac{1}{2} \Delta \Gamma_{D} t\right)\left|\hat{\rho}_{\text {wrong }}\right|^{2}+\frac{1}{4}\left(\Delta m_{D} t\right)^{2}-\frac{1}{2} \Delta \Gamma_{D} t \operatorname{Re} \frac{p}{q} \hat{\rho}_{\text {wrong }}+\Delta m_{D} t \operatorname{Im} \frac{p}{q} \hat{\rho}_{\text {wrong }}\right],}
\end{gathered}
$$

where

$$
\hat{\rho}_{\text {wrong }} \equiv \frac{T\left(D^{0} \rightarrow \ell^{-} X\right)}{T\left(D^{0} \rightarrow \ell^{+} X\right)},
$$

denotes the ratio of $\Delta C=\Delta Q$ to $\Delta C=-\Delta Q$ amplitudes,

$$
\left|D_{1,2}\right\rangle=p\left|D^{0}\right\rangle \pm q\left|\bar{D}^{0}\right\rangle
$$

relates mass and flavor eigenstates, and the oscillating functions multiplying the usual $e^{-t / \tau_{D}}$ term have been expanded in powers of the proper time $t$ since $x_{D}, y_{D} \ll 1$. The $\Delta C=\Delta Q$ term has no $t$ dependence beyond that of $e^{-t / \tau_{D}}$, the pure oscillation term has a $t^{2}$ dependence, while the interference between the two generates a term linear in $t$.

The violation of the $\Delta Q=-\Delta C$ rule arises even within the Standard Model for the decays $D^{0} \rightarrow K^{+} \pi^{-}$due to doubly Cabibbo suppressed transitions (DCST) producing the direct decay $D^{0} \rightarrow K^{+} \pi^{-}$, with a branching ratio $\operatorname{Br}\left(D^{0} \rightarrow K^{+} \pi^{-}\right)=(2.8 \pm 0.9) \pm 10^{-4}[110,122]$, and thus mimicking the signal for $D \bar{D}$ mixing. The equation corresponding to Eq. 120 is:

$$
\Gamma\left(D^{0}(t) \rightarrow K^{+} \pi^{-}\right) \propto e^{-\Gamma_{D^{0}} t} \tan ^{4} \theta_{C}\left|\hat{\rho}_{K \pi}\right|^{2}
$$




$$
\begin{gathered}
\times\left[1+\frac{1}{2} \Delta \Gamma_{D} t+\frac{\left(\Delta m_{D} t\right)^{2}}{4 \tan ^{4} \theta_{C}\left|\hat{\rho}_{K \pi}\right|^{2}}-\frac{\Delta \Gamma_{D} t}{2 \tan ^{2} \theta_{C}\left|\hat{\rho}_{K \pi}\right|} \operatorname{Re}\left(\frac{p}{q} \frac{\hat{\rho}_{K \pi}}{\left|\hat{\rho}_{K \pi}\right|}\right)\right. \\
\left.+\frac{\Delta m_{D} t}{\tan ^{2} \theta_{C}\left|\hat{\rho}_{K \pi}\right|} \operatorname{Im}\left(\frac{p}{q} \frac{\hat{\rho}_{K \pi}}{\left|\hat{\rho}_{K \pi}\right|}\right)\right],
\end{gathered}
$$

where

$$
\tan ^{2} \theta_{C} \cdot \hat{\rho}_{K \pi} \equiv \frac{T\left(D^{0} \rightarrow K^{+} \pi^{-}\right)}{T\left(D^{0} \rightarrow K^{-} \pi^{+}\right)}
$$

is the fraction of wrong-sign decays.

One can also search for lifetime differences in certain well-chosen $D^{0}$ decay channels in order to probe the contributions to oscillations from the $y_{D}$ term of Eq. 111. With $C P$ invariance holding (at least) to good approximation, $C P$ eigenstates can be treated as mass eigenstates. While $D^{0} \rightarrow K^{+} K^{-}, \pi^{+} \pi^{-}$ will then exhibit $\Gamma_{+}, D^{0} \rightarrow K_{S} \phi, K_{S} \omega, K_{S} \rho, K_{S} \eta$ etc. will be controlled by $\Gamma_{-}$, where $\Gamma_{+}\left[\Gamma_{-}\right]$denotes the width for the $C P$ even [odd] state and

$$
\Delta \Gamma=\Gamma_{+}-\Gamma_{-}
$$

Furthermore the width for $D^{0} \rightarrow K^{-} \pi^{+}$is approximately given by $\left(\Gamma_{+}+\Gamma_{-}\right) / 2$ $[111,112]$.

\subsubsection{CP Violation in D Decays}

There is a wide field of potential $C P$ violation in $D$ decays that can be discussed in close qualitative analogy to $B$ decays.

$C P$ asymmetries that necessarily involve $D^{0}-\bar{D}^{0}$ oscillations can arise in final states that are $C P$ eigenstates, like $K^{+} K^{-}$or $\pi^{+} \pi^{-}$:

$$
\begin{aligned}
\Gamma\left(D^{0}(t)\right. & \left.\rightarrow K^{+} K^{-}\right) \propto e^{-\Gamma_{D} t}\left(1+\sin \Delta m_{D} t \cdot \operatorname{Im} \frac{q}{p} \bar{\rho}_{K^{+} K^{-}}\right) \\
& \simeq e^{-\Gamma_{D} t}\left(1+\frac{\Delta m_{D} t}{\Gamma_{D}} \cdot \frac{t}{\tau_{D}} \cdot \operatorname{Im} \frac{q}{p} \bar{\rho}_{K^{+} K^{-}}\right) .
\end{aligned}
$$

With $\left.x_{D}\right|_{S M} \leq 10^{-2}$ and $\left.\operatorname{Im} \frac{q}{p} \bar{\rho}_{K^{+} K^{-}}\right|_{K M} \sim \mathcal{O}\left(10^{-3}\right)$, one arrives at an asymmetry of only around $10^{-5}$, which would likely be too small to measure even at a $\nu \mathrm{MC}$. Yet with new physics one conceivably has $\left.x_{D}\right|_{N P} \leq 0.1$ and $\left.\operatorname{Im} \frac{q}{p} \bar{\rho}_{K^{+} K^{-}}\right|_{N P} \sim \mathcal{O}\left(10^{-1}\right)$, leading to an asymmetry that could be as large as of order $1 \%$. 
Likewise, one can search for CP violation by comparing the proper time distribution of Eq. 123 for the doubly Cabibbo suppressed transitions $D^{0} \rightarrow K^{+} \pi^{-}$ with that for $\bar{D}^{0}$ decays:

$$
\begin{gathered}
\Gamma\left(\bar{D}^{0}(t) \rightarrow K^{-} \pi^{+}\right) \propto e^{-\Gamma_{D^{0}} t} \tan ^{4} \theta_{C}\left|\hat{\bar{\rho}}_{K \pi}\right|^{2} \\
\times\left[1+\frac{1}{2} \Delta \Gamma_{D} t+\frac{\left(\Delta m_{D} t\right)^{2}}{4 \tan ^{4} \theta_{C}\left|\hat{\bar{\rho}}_{K \pi}\right|^{2}}-\frac{\Delta \Gamma_{D} t}{2 \tan ^{2} \theta_{C}\left|\hat{\bar{\rho}}_{K \pi}\right|} \operatorname{Re}\left(\frac{q}{p} \frac{\hat{\bar{\rho}}_{K \pi}}{\left|\hat{\bar{\rho}}_{K \pi}\right|}\right)\right. \\
\left.+\frac{\Delta m_{D} t}{\tan ^{2} \theta_{C}\left|\hat{\bar{\rho}}_{K \pi}\right|} \operatorname{Im}\left(\frac{q}{p} \frac{\hat{\bar{\rho}}_{K \pi}}{\left|\hat{\bar{\rho}}_{K \pi}\right|}\right)\right]
\end{gathered}
$$

where

$$
\tan ^{2} \theta_{C} \cdot \hat{\bar{\rho}}_{K \pi} \equiv \frac{T\left(\bar{D}^{0} \rightarrow K^{-} \pi^{+}\right)}{T\left(\bar{D}^{0} \rightarrow K^{+} \pi^{-}\right)} .
$$

In such new physics scenarios one would expect a considerably enhanced asymmetry - perhaps as large as $1 \% / \tan ^{2} \theta_{C} \sim 20 \%$ - but at the cost of smaller statistics. Hoping for an asymmetry of several percent is more realistic, though. Effects of that size would unequivocally signal the intervention of new physics! One should note that these rough estimates are based on $x_{D} \simeq 10^{-2}$ which would correspond to $r_{D} \simeq 10^{-4}$. This implies that, even if oscillations have not been found on the $r_{D}=10^{-4}$ level in semileptonic $D^{0}$ decays, a $C P$ asymmetry of several percent (or conceivably ten percent) could still be encountered in $D^{0} \rightarrow K^{+} \pi^{-}$!

Direct CP violation can occur as well. There are actually two types of effects: differences between partial rates for $C P$ conjugate transitions

$$
A_{C P}=\frac{\Gamma(D \rightarrow f)-\Gamma(\bar{D} \rightarrow \bar{f})}{\Gamma(D \rightarrow f)+\Gamma(\bar{D} \rightarrow \bar{f})}
$$

and asymmetries in final state distributions such as, e.g., Dalitz plot populations.

Strong final state interactions play an important part in both cases: in the former they must induce the phase shifts that are essential to make a difference observable; in the latter they can very significantly affect the observable asymmetry. The existence of resonances in the neighborhood of the charmed meson mass is proof that hadron dynamics is active in this energy region and will affect the weak decays of charmed particles.

The good news is that whenever there are $C P$ violating weak phases one can count on final state interactions to make them observable. The bad news 
is that interpreting a signal as evidence for new physics will pose a highly nontrivial theoretical challenge.

The Standard Model with the CKM ansatz can induce direct $C P$ asymmetries only in Cabibbo suppressed channels. Model-dependent estimates usually predict direct $C P$ asymmetries to be of the order of $10^{-3}$ but, exceptionally, they could reach the $10^{-2}$ level [123]. A measurement of the branching ratios for all related channels - in particular also those with neutral hadrons in the final state, as sketched above - would enable us to constrain the strong phase shifts quite significantly. A $\nu \mathrm{MC}$ will have a significant advantage in this respect!

\subsubsection{T Odd Correlations in $\Lambda_{c}$ Decays}

One special feature of $\nu \mathrm{MCs}$ is represented by the production of $\Lambda_{c}$ in $\mathrm{CC}$ and $\mathrm{NC}$ reactions:

$$
\nu N \rightarrow \nu \Lambda_{c} X \text { or } \mu \Lambda_{c} X
$$

This allows novel studies of various $\Lambda_{c}$ decay form factors with a $Q^{2}$ range extending to well above $m_{\Lambda_{c}}^{2}$. Yet even more intriguing and promising would be a detailed analysis of the final state in its semileptonic decays:

$$
\Lambda_{c}^{+} \rightarrow \ell^{+} \nu_{\ell} \Lambda
$$

With the parent $c$ quark being left-handed one expects the $\Lambda_{c}$ to emerge in a highly polarized state. The usual valence quark description actually suggests that the $\Lambda_{c}$ polarization is completely carried by its $c$ quark; i.e., a left-handed $c$ quark fragments into a left-handed $\Lambda_{c}$. Yet even with unpolarized $\Lambda_{c}$ one can form an experimentally observable $T$ odd correlation

$$
C_{T+-} \equiv\left\langle\vec{\sigma}_{\Lambda} \cdot\left(\vec{p}_{\Lambda} \times \vec{p}_{\ell}\right)\right\rangle
$$

connecting spin and momentum of the daughter hyperon with the lepton momentum.

In a general experimental process, observing a non-vanishing value for a $T$ odd correlation does not automatically establish that $T$ (and $C P$ ) invariance is violated since in general final state interactions could fake such an effect. However, this problem does not occur for Eq. 131 since it cannot be affected by either strong or electromagnetic final state interactions! This is analogous to the well known situation in $K^{+} \rightarrow \mu^{+} \nu \pi^{0}$ ( vs. $K_{L} \rightarrow \mu^{+} \nu \pi^{-}$). Like there, the CKM ansatz cannot generate an observable effect here, yet certain new physics scenarios can. An effect of order $10^{-2}$ is not inconceivable, particularly if the channel $\Lambda_{c} \rightarrow \tau \nu \Lambda$ could be studied [124]. 


\subsection{Summary on Charm Decay Physics at $\nu M C s$}

The research program at a $\nu \mathrm{MC}$ is likely to improve our knowledge and understanding of charm decays quite significantly even ten years from now:

- It would fill out many white spots on our map of $D, \Lambda_{c}, \Xi_{c}$ and $\Omega_{c}$ decays by measuring many new relative and absolute branching ratios, including for final states with more than one neutral particle.

- It would allow the measurement of $D^{+}, D_{s}^{+} \rightarrow \mu \nu, \tau \nu$ in a very clean environment.

- It could probe for $D^{0}-\bar{D}^{0}$ oscillations and for $C P$ asymmetries involving them with superbly clean systematics. It would significantly improve on the sensitivity that can be obtained at $B$ factories for such phenomena.

- It would enable us to search for direct $C P$ asymmetries in many different channels and at the same time provide us with information that could help us in properly interpreting a signal.

\section{Neutrino Oscillation Experiments with a Muon Storage Ring/Neutrino Factory}

\subsection{Status of Neutrino Oscillations at the Time of $\nu M C s$}

In a modern theoretical context, one generally expects nonzero neutrino masses and associated lepton mixing [9]. Experimentally, there has been accumulating evidence for such masses and mixing. All solar neutrino experiments (Homestake, Kamiokande, SuperKamiokande, SAGE, and GALLEX) show a significant deficit in the neutrino fluxes coming from the Sun [125]. This deficit can be explained by oscillations of the $\nu_{e}$ 's into one or more other weak eigenstates, with $\Delta m_{\text {sol }}^{2}$ of the order $10^{-5} \mathrm{eV}^{2} / c^{4}$ for solutions involving the MikheevSmirnov-Wolfenstein (MSW) resonant matter oscillations [126,127] or of the order of $10^{-10} \mathrm{eV}^{2} / c^{4}$ for vacuum oscillations. Accounting for the data with vacuum oscillations (VO) requires almost maximal mixing. The MSW solutions include one for small mixing angles (SMA) and one with essentially maximal mixing (LMA).

Another piece of evidence for neutrino oscillations is the atmospheric neutrino anomaly, observed by Kamiokande [128], IMB [129], SuperKamiokande [130] with the highest statistics, and by Soudan [131] and MACRO [132]. This data can be fit by the inference of $\nu_{\mu} \rightarrow \nu_{x}$ oscillations with $\Delta m_{a t m}^{2} \sim 3.5 \times 10^{-3}$ $\mathrm{eV}^{2} / c^{4}[130]$ and maximal mixing $\sin ^{2} 2 \theta_{\text {atm }}=1$. The identification $\nu_{x}=\nu_{\tau}$ is preferred over $\nu_{x}=\nu_{\text {sterile }}$ at about the $2.5 \sigma$ level [133], and the identifica- 
tion $\nu_{x}=\nu_{e}$ is excluded by both the SuperKamiokande data and the Chooz experiment $[134,135]$.

In addition, the LSND experiment [136] has reported observing $\bar{\nu}_{\mu} \rightarrow \bar{\nu}_{e}$ and $\nu_{\mu} \rightarrow \nu_{e}$ oscillations with $\Delta m_{L S N D}^{2} \sim 0.1-1 \mathrm{eV}^{2} / c^{4}$ and a range of possible mixing angles, depending on $\Delta m_{L S N D}^{2}$. This result is not confirmed, but also not completely ruled out, by a similar experiment, KARMEN [137]. Inclusion of the signal reported by LSND with the other two pieces of evidence would imply three distinct mass differences and hence four neutrinos. Some proposals for the form of the mixing matrix invoke only 3 generations of neutrinos to account for all signatures, while others invoke a fourth sterile neutrino.

A number of fits have been made to the existing neutrino data. The fit by the SuperKamiokande collaboration to its data yields a minimum in the $\chi^{2}$ at $\sin ^{2}\left(2 \theta_{a t m}\right)=1$, with an allowed region of $0.8 \lesssim \sin ^{2}\left(2 \theta_{a t m}\right) \lesssim 1$. In terms of the basic angles in the lepton mixing matrix, this implies that $\theta_{23}$ is close to $\pi / 4$ and allows a small, nonzero $\theta_{13}$, consistent with the bound from CHOOZ. As will be discussed below, a major physics capability of the muon storage ring/neutrino factory is the ability to measure $\theta_{13}$.

There are currently intense efforts to confirm and extend the evidence for neutrino oscillations in all of the various sectors - solar, atmospheric and accelerator. Some of these experiments are running; in addition to SuperKamiokande and Soudan-2, these include the Sudbury Neutrino Observatory, SNO, and the $\mathrm{K} 2 \mathrm{~K}$ long baseline experiment between KEK and Kamioka. Others are in development and testing phases, such as BOONE, MINOS, the CERN-Gran Sasso program, KAMLAND, and Borexino [138]. Among the long baseline neutrino oscillation experiments, the approximate distances are $L \simeq 250 \mathrm{~km}$ for K2K, $730 \mathrm{~km}$ for both MINOS, from Fermilab to Soudan and the proposed CERN-Gran Sasso experiments. The sensitivity of these experiments is projected to reach down roughly to the level $\Delta m^{2} \sim 10^{-3} \mathrm{eV}^{2} / c^{4}$. Experiments that are planned as part of this program include [139] ICANOE and OPERA [140]. Although they are expected to begin operation after MINOS, they will involve somewhat different detector designs and plan to focus on establishing $\tau$ appearance. This, then, is the program of research for the next several years.

\subsection{Oscillation Experiments at $\nu M C s$}

Although a neutrino factory based on a muon storage ring will turn on several years after this near-term period in which K2K, MINOS, and the CERN-Gran Sasso experiments will run, we believe that it has a valuable role to play, given the very high-intensity neutrino beams of fixed flavor-pure content, including, in particular, $\nu_{e}$ and $\bar{\nu}_{e}$ beams as well as the conventional $\nu_{\mu}$ and $\bar{\nu}_{\mu}$ beams. 
The potential of the neutrino beams from a muon storage ring is that, in contrast to a conventional neutrino beam $\pi^{+} / K^{+}$decay, is primarily $\nu_{\mu}$ with some admixture of $\nu_{e}$ 's and other flavors from $K$ decays, the neutrino beams from the muon storage ring would high extremely high purity: $\mu^{-}$beams would yield $50 \% \nu_{\mu}$ and $50 \% \bar{\nu}_{e}$, and so forth for the charge conjugate case of $\mu^{+}$ beams. Furthermore, these could be produced with extremely high intensities, of order $10^{20}$ to $10^{21}$ neutrinos per year.

Given the form of the oscillation probabilities, a neutrino beam for an oscillation experiment would optimally be made from the lower end of the spectrum of energies considered in this report. Because of the lower requirements on beam focusing and acceleration, making a muon storage ring for the purposes of an oscillation experiment has been proposed as a first step towards developing a muon collider. Energies being considered range from $20 \mathrm{GeV}$ to $50 \mathrm{GeV}$, and the geometry of the final muon ring is very different from a traditional collider ring, in that the straight section that points to a neutrino experiment comprises between 25 and $40 \%$ of the "circumference" of the ring.

The types of neutrino oscillations that can be searched for with the neutrino factory based on a muon storage ring, along with the final state charged lepton species for neutrino-nucleon DIS that tags the interacting neutrino flavor, are listed below for the case of the $\nu_{\mu} \bar{\nu}_{e}$ beam from $\mu^{-}$, decaying as $\mu^{-} \rightarrow \nu_{\mu} e^{-} \bar{\nu}_{e}$ :

(1) $\nu_{\mu} \rightarrow \nu_{\mu}, \nu_{\mu} \rightarrow \mu^{-}$(survival);

(2) $\nu_{\mu} \rightarrow \nu_{e}, \nu_{e} \rightarrow e^{-}$(appearance);

(3) $\nu_{\mu} \rightarrow \nu_{\tau}, \nu_{\tau} \rightarrow \tau^{-}, \tau^{-} \rightarrow\left(e^{-}, \mu^{-}\right) \ldots$ (appearance* a $^{*}$

(4) $\bar{\nu}_{e} \rightarrow \bar{\nu}_{e}, \bar{\nu}_{e} \rightarrow e$ (survival);

(5) $\bar{\nu}_{e} \rightarrow \bar{\nu}_{\mu}, \bar{\nu}_{\mu} \rightarrow \mu^{+}$(appearance);

(6) $\bar{\nu}_{e} \rightarrow \bar{\nu}_{\tau}, \bar{\nu}_{\tau} \rightarrow \tau^{+} ; \tau^{+} \rightarrow\left(e^{+}, \mu^{+}\right) \ldots$ (appearance* ${ }^{*}$;

where the asterisks denote that the tau appearance signatures may be somewhat indirect in involving detection of the leptonic daughters of tau decays rather than the decay vertices of the taus themselves.

It is clear from the list of processes above that, since the beam contains both neutrinos and antineutrinos, the only way to determine what the parent neutrino was is to measure the charge of the final state lepton. The $\nu_{\mu} \rightarrow \nu_{e}$ oscillation will produce a wrong-sign $e^{-}$as will the $\nu_{\mu} \rightarrow \nu_{\tau}$ oscillation followed by $\tau$ decay to $e^{-}$. The easiest wrong-sign lepton signatures to detect arise from the oscillations $\bar{\nu}_{e} \rightarrow \bar{\nu}_{\mu}$, giving a $\mu^{+}$, and from $\bar{\nu}_{e} \rightarrow \bar{\nu}_{\tau}$, giving a $\tau^{+}$which will decay part of the time to $\mu^{+}$. If one is searching for $\tau$ final states, muon storage ring energies above $30 \mathrm{GeV}$ should be used to minimize the threshold kinematic suppression.

To get a rough idea of how the sensitivity of an oscillation experiment would scale with energy and baseline length, recall that the event rate in the absence 
of oscillations is simply the neutrino flux times the cross section. First of all, neutrino cross sections in the region above about $10 \mathrm{GeV}$ (and slightly higher for $\tau$ production) grow linearly with the neutrino energy. Secondly, the beam divergence is a function of the initial muon storage ring energy; this divergence yields a flux, as a function of $\theta_{d}$, the angle of deviation from the forward direction, that goes like $1 / \theta_{d}^{2} \sim E^{2}$. Combining this with the linear $E$ dependence of the neutrino cross section and the overall $1 / L^{2}$ dependence of the flux far from the production region, one finds that the event rate goes like

$$
\frac{d N}{d t} \sim \frac{E^{3}}{L^{2}}
$$

To set the scale, consider an experiment that sees $2 \times 10^{20} 30 \mathrm{GeV}$ muon decays in a straight section pointed at a detector $2800 \mathrm{~km}$ away. In the absence of oscillations, the $\nu_{\mu}\left(\bar{\nu}_{e}\right)$ charged current rate would be $52,500(22,600)$ events per 10 kton [7]. Figure 16 shows the relative $\nu_{e}$ and $\bar{\nu}_{\mu}$ statistics for two configurations: one is a $20 \mathrm{GeV} \mu^{+}$ring with a detector at $2800 \mathrm{~km}$, the other is a $50 \mathrm{GeV} \mu^{+}$ring with a detector at $9100 \mathrm{~km}$, which is close to the distance from either Fermilab or CERN to Kamiokande.

Now recall the general formula for the probability of a two-species neutrino oscillation in vacuum, say of $\nu_{e} \rightarrow \nu_{\mu}$ :

$$
\begin{aligned}
P\left(\nu_{e}\right. & \left.\rightarrow \nu_{\mu}\right)=4\left|U_{13}\right|^{2}\left|U_{23}\right|^{2} \sin ^{2}\left(\frac{\Delta m_{a t m}^{2} L}{4 E}\right) \\
& =\sin ^{2}\left(2 \theta_{13}\right) \sin ^{2}\left(\theta_{23}\right) \sin ^{2}\left(\frac{\Delta m_{a t m}^{2} L}{4 E}\right) .
\end{aligned}
$$

Where the $\sin ^{2}$ term is small, it can be expanded to give a factor of $(L / E)^{2}$ that cancels the $L$ dependence in equation 133 and reduces the $E$ dependence to a linear factor. Underlying these considerations of optimal energy is the fact that, even if one designs an experiment for $\tau$ appearance, the overall event rate of detected $\tau$ 's may be rather small.

It is quite likely that, by the time a neutrino factory turns on, $\Delta m_{a t m}^{2}$. and $\sin ^{2}\left(2 \theta_{23}\right)$ will be known at the $10-30 \%$ level. Although a neutrino factory could undoubtedly improve the precision on those two parameters, the novel physics that can be addressed is a determination of $\theta_{13}$, and the sign of $\Delta m_{a t m}^{2}$. By using matter effects, and a comparison of $\nu_{a}$ versus $\bar{\nu}_{a}$ oscillations (by switching the muon storage ring from $\mu^{-}$to $\mu^{+}$), the sign of $\Delta m_{\text {atm. }}^{2}$ can be determined. 

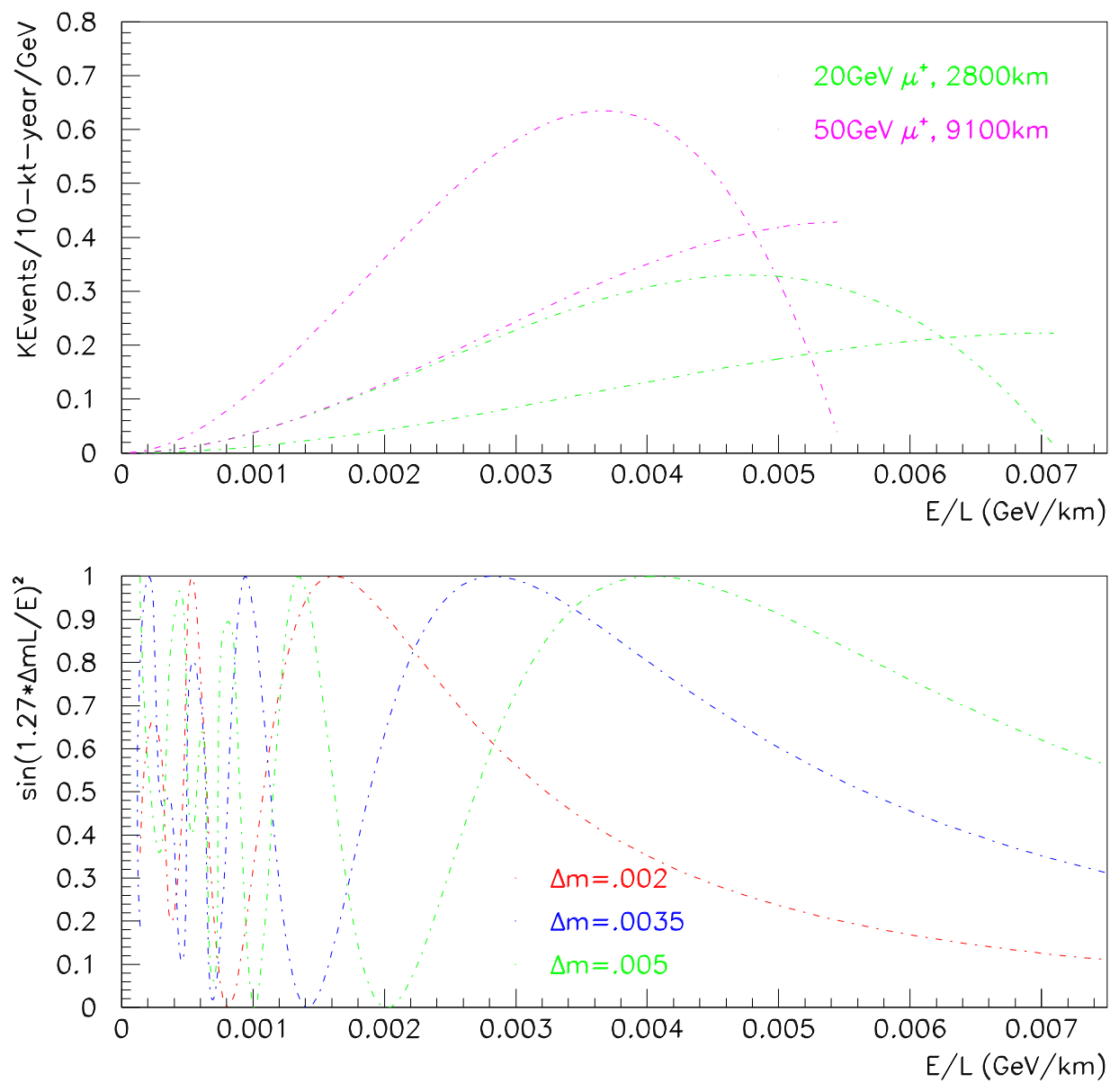

Fig. 16. The upper plot shows $\nu_{e}$ and $\bar{\nu}_{\mu}$ event rates per $\mathrm{GeV}$ as a function of $\mathrm{E} / \mathrm{L}$ for two different experiments. The lower plot shows the $\mathrm{L} / \mathrm{E}$ dependence of the oscillation probability, assuming the largest $\delta m^{2}$ is $2,3.5$, or $5 \times 10^{-3} \mathrm{eV}^{2} / c^{4}$.

\subsection{Matter Effects}

With the advent of the muon storage ring, the distances one can place detectors will become large enough that, for the first time, matter effects can be exploited in accelerator-based oscillation experiments. Simply put, matter effects are the matter-induced oscillations which neutrinos undergo along their flight path through the Earth from the source to the detector. Given the typical density of the earth, matter effects are important for the neutrino energy range $E \sim O(10) \mathrm{GeV}$ and $\Delta m_{\text {atm }}^{2} \sim 10^{-3} \mathrm{eV}^{2} / c^{4}$ values that are relevant for $\nu \mathrm{MC}$ long baseline experiments.

Follow-up studies to initial discussion [126] of matter-induced resonant neu- 
trino oscillations include an early study of these effects that assumed three neutrino generations [141] and a discussion of the sensitivity of an atmospheric neutrino experiment to small $\Delta m^{2}$ due to the long baselines and the necessity of taking into account matter effects was discussed[142]. Many analyses were performed in the 1980's of the effects of resonant neutrino oscillations[127] on the solar neutrino flux, and matter effects in the Earth [143,144], and of matter effect on atmospheric neutrinos[145]. Work continues[146,147] on matter effects relevant to atmospheric neutrinos. Early studies of matter effects on long baseline neutrino oscillation experiments [148] have been extended to cover neutrino factories[7,149], [150,151]

In recent papers by one of the authors $(\mathrm{RS})$ and I. Mocioiu, calculations were presented of the matter effect for parameters relevant to the possible long baseline neutrino experiments envisioned for the muon storage ring/neutrino factory $[152,153]$. In particular, these authors compared the results obtained with constant matter density along the neutrino path versus results obtained by incorporating the actual density profiles. They studied the dependence of the oscillation signal on both $E / \Delta m_{a t m}^{2}$. and on the angles in the leptonic mixing matrix, and commented on the influence of $\Delta m_{\text {sol. }}^{2}$ and $\mathrm{CP}$ violation on the oscillations. Additional recent studies are listed in the references $[154,155]$.

\subsection{Detector Considerations}

In order to measure oscillation parameters that describe the transitions above, one would ideally want a detector that could identify the existence and flavor of any outgoing lepton from the neutrino interaction, as well as the hadronic and leptonic energy in the event. When measuring very small oscillation probabilities, however, backgrounds must be taken into account. Naively one would think that simply detecting a muon of opposite charge to that in the storage ring is a signal for the electron neutrino oscillating. However, pions and kaons are produced copiously in neutral and charged current neutrino interactions, and if one decays to a muon before it interacts in the detector this can constitute a significant background. At higher energies charmed mesons are also produced which decay immediately to muons $10 \%$ of the time. Ultimately, detectors will need to be designed that have sufficient resolution on both the energies and the angles of the final state lepton and hadronic shower to be able to remove these backgrounds.

Although detectors exist that could identify all of the final state leptons and their charges, the challenge is to make them on the several kiloton scale. If the largest $\Delta m^{2}$ is in the LSND region, there will undoubtedly be more work done to optimize relatively low mass detectors that emphasize tau appearance. However, the only detectors which have thus far been proposed on the 10-40 
kiloton scale are for detecting wrong sign muon events. The two detector technologies that have been considered in detail for oscillation experiments for a muon storage ring will now be discussed in turn: one is a magnetized sampling calorimeter such as the one used by MINOS [156] and the other is a liquid argon time projection chamber (TPC) combined with a muon spectrometer such as the one proposed by the ICANOE [157] collaboration for the CERN to Gran Sasso neutrino beam.

\subsubsection{Magnetized Sampling Calorimeters}

Magnetized sampling calorimeters consist simply of alternating layers of magnetized steel and readout, where the readout traditionally consists of scintillator and/or drift chambers.

The charged particle efficiency of the readout planes can be close to $100 \%$ so the performance of the sampling calorimeter depends primarily on the sampling frequency of the detector. A steel/scintillator sandwich with sampling every $5 \mathrm{~cm}$ of steel would have a fractional hadron energy $(H)$ resolution of approximately [156]

$$
H \equiv \frac{\sigma_{E}}{E_{h a d}} \simeq \frac{0.76}{\sqrt{E_{h a d}[G e V]}}
$$

With fine enough transverse segmentation, the hadron angular resolution is dominated by the hadron energy resolution. The muon energy and angular resolution are expected to be much better than for the hadronic shower.

Although separating $\nu_{e}$ charged current events from neutral current events is difficult and determining the charge of the outgoing electron impossible in this detector, a muon in the final state can be easily and efficiently detected, and its charge, momentum, and initial outgoing angle can be determined once the muon traverses enough steel to be spatially separated from the hadronic shower. Kinematic cuts can be made on the muon momentum and its component transverse to the hadronic shower to reduce the background from charm production. With signal efficiencies from 25 to 30\%, the backgrounds can be reduced to a level of $10^{-5}$ to $10^{-6}$, depending on the neutrino energy. At higher energies the backgrounds are larger but the faster improvement in the background rejection actually causes a reduction in the background contributions to the analysis [158].

\subsubsection{Liquid Argon TPCs}

The ICANOE-type detector would consist of a large volume of liquid argon instrumented with time projection chambers (TPC's), followed by a much thin- 
ner volume of magnetized steel where a muon's charge and momentum can be determined. The TPC would have very small wire spacing $(3 \mathrm{~mm})$ and would act much like an electronic bubble chamber. Electron neutrino charged current interactions could be distinguished from neutral current interactions, although the electron charge could not be measured. By breaking up the event samples into four distinct classes - right sign muons, wrong sign muons, electron-like events and neutral current events - one could fit all four distributions simultaneously to determine oscillation parameters. $\nu_{\tau}$ 's might also be identified on a statistical basis by looking at the acoplanarity distribution in the event sample.

The energy and angular resolutions of all the final state particles would be extremely good, e.g.,

$$
\frac{\sigma_{H}}{E_{H}} \simeq \frac{0.20}{\sqrt{E_{h a d}[G e V]}}
$$

for the hadronic energy and $150 \mathrm{mrad}$ for hadron shower angles. However, the ability of this detector to see wrong-sign muons would depend primarily on the segmentation between the liquid argon and the magnetized spectrometer and on the thickness of the spectrometer itself. The thinner one makes the spectrometer, the more likely one is to have backgrounds from charge misidentification. The thicker the spectrometer, the less room there is for the liquid argon in a given volume. The thinner the liquid argon, the higher the acceptance for low energy muons (since muons lose approximately $210 \mathrm{MeV} / \mathrm{m}$ in liquid argon [159]), but the less target volume one has overall. Clearly optimization of this geometry is needed, and will depend somewhat on the energy of the muon storage ring.

\subsubsection{Muon Detector Conclusions}

Although the two types of detectors have different strengths, detailed studies $[160,149,150,153,161,3,4]$ have shown that both either would be adequate to make precise measurements of $\left|\delta m^{2}\right|, \operatorname{sign}\left(\delta m^{2}\right)$, and $\sin ^{2} \theta_{23}$; and to extend the sensitivity of $\sin ^{2} \theta_{13}$ by 1-2 orders of magnitude in the scenario where the largest $\Delta m^{2}$ is described by the atmospheric neutrino anomaly.

\subsubsection{Tau and Electron Detectors}

Alternate technologies must be employed to achieve electron or tau identification event-by-event, or electron or tau charge measurements. If LSND is confirmed and the largest $\Delta m^{2}$ would suggest baselines on the order of tens of $\mathrm{km}$, then a much higher premium will be placed on designing detectors that can do tau and electron charge determination, and they will not have 
to be as massive. At these short baselines, detectors on the 1 kiloton scale could be quite adequate to make precision measurements on $\nu_{e} \rightarrow \nu_{\tau}$ and $\nu_{\mu} \rightarrow \nu_{\tau}$. Even if LSND is not confirmed, efforts to make massive tau and electron charge identification detectors should not be abandoned since these two channels still comprise a large part of the mixing matrix and should be researched to confirm our understanding of neutrino mixing.

One category of new detectors uses thin $(\sim 100 \mu \mathrm{m})$ sheets of emulsion combined with low-density $(\sim 300 \mu \mathrm{m})$ spacers, and thin sheets of metal to give the detector mass. With emulsion one can measure the kink that occurs when a tau decays by comparing the slope of a track before and after the spacer. Such a geometry, with lead as the mass, is described in reference [162]. This would be very useful for identifying taus and electrons. However, for charge identification one needs to introduce a magnetic field. This could be done using an extremely large external magnet, such as the one used in ATLAS, and thin steel plates, or by using a coil and magnetized steel to make the mass [163]. Since the overhead for analysis of each event is high in this sort of detector, one would place it in a region where the tau appearance probability is maximized.

\subsection{Conclusions on Neutrino Oscillation Studies at $\nu M C s$}

In conclusion, neutrino masses and mixing are generic theoretical expectations. The seesaw mechanism naturally yields light neutrinos, although its detailed predictions are model-dependent and may require a lower mass scale than the GUT mass scale. Current atmospheric neutrino data is consistent with maximal mixing in the relevant channel, which at present is favored to be $\nu_{\mu} \rightarrow \nu_{\tau}$. Even after the near-term program of experiments by K2K, MINOS, the CERN-Gran Sasso experiments, and mini-BOONE, a high-intensity neutrino factory generating $10^{20}-10^{21}$ neutrinos per year will add greatly to our knowledge of the neutrino masses and mixing matrix. Ideally, the muon storage ring should be coupled with two long-baseline neutrino oscillation experiments, located at different baselines, that can take advantage of matter effects to amplify certain transitions and with a massive detector that will identify $\mu$ 's and $\tau$ 's with charge discrimination. In particular, it should be able to measure $\Delta m_{\text {atm. }}^{2}$ and $\sin ^{2}\left(2 \theta_{23}\right)$ to the level of several per cent and also give important information about the sign of $\Delta m_{a t m}^{2}$. and about $\sin ^{2}\left(2 \theta_{13}\right)$. 


\section{Summary}

Beams from $\nu \mathrm{MCs}$ have the potential to provide vast improvements over today's conventional neutrino beams from $\pi / K$ decays. They are much more intense, have a much smaller transverse extent and produce precisely predictable beam spectra.

Oscillation experiments may extend even to intercontinental baselines, while unprecedented event statistics approaching of order $10^{9}$ to $10^{10}$ precisely constructed DIS events will open new regimes of neutrino interaction physics. The extraordinary rates will enable use of active vertexing targets surrounding by a high resolution spectrometer and calorimeter. The first polarized targets for neutrino scattering can be substituted for special studies.

Highlights of $\nu \mathrm{MC}$ physics program include:

- substantially extending the reach of accelerator-based experiments to study neutrino oscillations;

- measurements of the CKM quark mixing matrix elements $\left|V_{c d}\right|,\left|V_{u b}\right|,\left|V_{c s}\right|$, and $\left|V_{c b}\right|$ in inclusive high $Q^{2}$ scattering, with few percent accuracies achievable for the first two;

- a realistic opportunity to determine the detailed quark-by-quark structure of the nucleon;

- mapping out the quark-by-quark spin structure with polarized targets, and, perhaps, determining the gluon contribution to the nucleon's spin;

- some of the most precise measurements and tests of perturbative QCD;

- tests of the electroweak theory through measurements of $\sin ^{2} \theta_{W}$ with fractional uncertainties approaching $10^{-4}$;

- a new realm to search for exotic physics processes;

- a charm factory with unique and novel capabilities;

- a new laboratory for the study of nuclear physics with neutrino beams.

The potential experimental capabilities of $\nu \mathrm{MCs}$ reach so far beyond present neutrino programs that physics surprises not touched upon in this report can be expected. To see, we must build the machines.

\section{Acknowledgments}

We thank Janet Conrad, Keith Ellis, Michelangelo Mangano, Michael Shaevitz and Don Summers for helpful information and comments. This work was performed under US Department of Energy Contract Numbers DE-AC0298CH10886, DE-AC02-76CH03000, DE-FG02-91ER40684, DE-FG02-91ER40685 
and DE-FG03-99ER41093, under US National Science Foundation Contract Numbers PHY 96-05080, NSF 97-22101, PHY 98-13383 and PHY 00-87419 and under the auspices of the Illinois Board of Higher Education.

\section{References}

[1] The Muon Collider Collaboration, Status of Muon Collider Research and Development and Future Plans, Phys. Rev. ST Accel. Beams, 3 August, 1999.

[2] The Muon Collider Collaboration, $\mu^{+} \mu^{-}$Collider: A Feasibility Study, BNL52503, Fermilab-Conf-96/092, LBNL-38946, July 1996.

[3] C. Albright et al., Physics at a Neutrino Factory, FERMILAB-FN-692, May 9, 2000. Available at

http://www.fnal.gov/projects/muon_collider/nu/study/study.html.

[4] Feasibility Study-II of a Muon-Based Neutrino Source, ed. S. Ozaki, R. Palmer, M. Zisman and J. Gallardo, June, 2001. Available at http://www . cap.bnl.gov/mumu/studyii/FS2-report.html.

[5] B.J. King, Assessment of the Prospects for Muon Colliders, paper submitted in partial fulfillment of requirements for Ph.D., Columbia University, New York (1994), available from http://xxx.lanl.gov/ as physics/9907027.

[6] B.J. King, Neutrino Physics at a Muon Collider, Proc. Workshop on Physics at the First Muon Collider and Front End of a Muon Collider, Fermilab, November 6-9, 1997, hep-ex/9907033.

[7] S. Geer, Phys. Rev. D 57, 6989 (1998) hep-ph/9712290.

[8] T. Bolton, "Heavy Quark Production in Deep Inelastic Neutrino Scattering", Kansas State University pre-print KSU-HEP-00-003 (2000).

[9] Ikaros Bigi et al., BNL-67404, May, 2000.

[10] A Feasibility Study of a Neutrino Source Based on a Muon Storage Ring, ed. Norbert Holtkamp and David Finley, March, 2000. Available at http://www.fnal.gov/projects/muon_collider/nu/study/report/machine_report/.

[11] R. Raja and A. Tollestrup, Phys. Rev. D 58, 013005 (1998) hep-ex/9801004.

[12] J. M. Conrad, M. H. Shaevitz and T. Bolton, Rev. Mod. Phys. 70, 1341 (1998).

[13] C. Johnstone, Private communication.

[14] B.J. King, Mighty MURINEs: Neutrino Physics at Very High Energy Muon Colliders, Proc. HEMC'99 Workshop - Studies on Colliders and Collider Physics at the Highest Energies: Muon Colliders at $10 \mathrm{TeV}$ to $100 \mathrm{TeV}$; Montauk, NY, September 27-October 1, 1999, hep-ex/0005007. 
[15] Particle Data Group, D. E. Groom et al., Eur. Phys. J. C15, 1 (2000); http://pdg.lbl.gov.

[16] H. Plothow-Besch, Comput. Phys. Commun. 75, 396 (1993).

[17] A. O. Bazarko et al. (CCFR Collaboration), Z. Phys. C 65, 189 (1995).

[18] M. Goncharov, et al. (NuTeV Collaboration), "Precise Measurement of Dimuon Production Cross-Sections in muon neutrino Fe and muon antineutrino Fe Deep Inelastic Scattering at the Tevatron", e-print number hep-ex/0102049, submitted to Phys. Rev. D., February, 2001.

[19] E. Eskut et al. (CHORUS Collaboration), Phys. Lett. B503, 1 (2001).

[20] A. Alton et al. (NuTeV Collaboration), "Observation of Neutral Current Charm Production in $\nu_{\mu}$ Fe Scattering at the Tevatron", hep-ex/0008068, to be published in Phys. Rev. D.

[21] A. A. Petrov and T. Torma, Phys. Rev. D 60, 093009 (1999) hep-ph/9906254.

[22] G. Altarelli and G. Parisi, Nucl. Phys. B 126, 298 (1977).

[23] Yu. L. Dokshitser, D.I. Diakonov, and S.I. Troian, Phys. Lett. 78 B, 290 (1978).

[24] Yu. L. Dokshitser, et al., Phys. Rep. 58, 269 (1980).

[25] V. N. Gribov and L. N. Lipatov, Sov. J. Nucl. Phys. 15, 438 (1972) .

[26] D. J. Gross and C. H. Llewellyn Smith, Nucl. Phys. B14, 337 (1969).

[27] J. Chyla and A. L. Kataev, Phys. Lett. B297, 385(1992).

[28] S. A. Larin and J. A. Vermaseren, Phys. Lett. B259, 345 (1991).

[29] V. M. Braun and A. V. Kolesnichenko, Nucl. Phys. B283, 723 (1987).

[30] G. G. Ross and R. G. Roberts, Phys. Lett. B322, 425 (1994) hep-ph/9312237.

[31] S. L. Adler, Phys. Rev. 143, 1144 (1966).

[32] W.G. Seligman, et al. (CCFR Collaboration), Phys. Rev. Lett. 79, 1213(1997)

[33] J. H. Kim, et al. (CCFR/NuTeV Collaboration), Phys. Rev. Lett. 81, 3595 (1998).

[34] H. Georgi and H. D. Politzer, Phys. Rev. D 14, 1829 (1976).

[35] M. A. Aivazis, J. C. Collins, F. I. Olness and W. Tung, energies," Phys. Rev. D 50, 3102 (1994) hep-ph/9312319.

[36] U. K. Yang and A. Bodek, Phys. Rev. Lett. 82, 2467 (1999) hep-ph/9809480.

[37] H. L. Lai et al. [CTEQ Collaboration], Eur. Phys. J. C12, 375 (2000) hepph/9903282.

[38] M. Virchaux, and A. Milsztajn, Phys. Lett. B 274, 221 (1992).. 
[39] H. Deden and et al. [Gargamelle Neutrino Collaboration], Nucl. Phys. B85, 269 (1975).

[40] X. Guo and J. Qiu, hep-ph/9810548.

[41] An ICE target will be used in the Laser Electron Gamma-ray Source (LEGS) experiment at Brookhaven National Laboratory, http://www. legs.bnl.gov.

[42] Deborah A. Harris and Kevin S. McFarland, A Small Target Neutrino DeepInelastic Scattering Experiment at the First Muon Collider, Proc. Workshop on Physics at the First Muon Collider and Front End of a Muon Collider, Fermilab, November 6-9, 1997, hep-ex/9804010.

[43] M.R. Adams, et al. (E665 Collaboration ), Nucl. Instrum. Meth. A291, 533 (1990).

[44] C. Boros, J. T. Londergan and A. W. Thomas, Phys. Rev. D 59, 074021 (1999) hep-ph/9810220.

[45] S. A. Kulagin, hep-ph/9812532.

[46] K. J. Eskola, V. J. Kolhinen, P. V. Ruuskanen and C. A. Salgado, Nucl. Phys. A661, 645 (1999) hep-ph/9906484.

[47] A. Benvenuti et al., Z. Phys. C63 (1994), 29.

[48] M. Vakili et al., PR D61 (2000).

[49] M. Vakili, et al. (CCFR Collaboration), Phys. Rev. D61, 052003 (2000).

[50] A concise review with a critical overview of the literature is given in: I. Bigi, M. Shifman and N. Uraltsev, Ann. Rev. Nucl. Part. Sci. 47, 591 (1997) hepph/9703290], with references to earlier work

[51] J. Goldman, A Next-to-Leading-Order QCD Analysis of Charged Current Event Rates in $\nu N$ Deep Inelastic Scattering at the Tevatron, Kansas State University Ph.D. thesis, unpublished (2000).

[52] P. Annis, et al. (CHORUS collaboration), Phys. Lett. B435, 458 (1998).

[53] T. Adams, et al. (NuTeV Collaboration), Phys. Rev. D 6, 092001 (2000).

[54] F. J. Hasert et al. [Gargamelle Neutrino Collaboration], Phys. Lett. B46, 138 (1973).

[55] H. Abramowicz et al., Phys. Rev. Lett. 57, 298 (1986). D. Bogert et al., Phys. Rev. Lett. 55, 1969 (1985). M. Jonker et al. [CHARM Collaboration], Phys. Lett. B99, 265 (1981).

[56] LEP Electroweak Working Group Summaries, see, e.g., CERN-EP-2000-016

[57] K. Abe et al. [SLD Collaboration], Phys. Rev. Lett. 78, 2075 (1997). K. Abe et al. [SLD Collaboration], "A high-precision measurement of the left-right Z boson cross-section asymmetry," hep-ex/0004026. 
[58] H. S. Chen and G. J. Zhou, Phys. Lett. B331, 441 (1994).

[59] D. Amidei, R. Brock, et al. "Report of the TeV2000 Study Group", FermilabPUB/96-082.

[60] S. C. Bennett and C. E. Wieman, Phys. Rev. Lett. 82, 2484 (1999).

[61] K. S. Kumar, E. W. Hughes, R. Holmes and P. A. Souder, Mod. Phys. Lett. A10, 2979 (1995).

[62] A. Czarnecki and W. J. Marciano, Int. J. Mod. Phys. A15, 2365 (2000) hepph/0003049, Phys. Rev. D 53, 1066 (1996) [hep-ph/9507420].

[63] S. Sarantakos, A. Sirlin and W. J. Marciano, Nucl. Phys. B217, 84 (1983).

[64] P. Villain et al., Phys. Lett. B335:246 (1994). See also Phys. Lett. B302:351 (1993) and Phys. Lett. B281:159 (1992).

[65] Private communications with W. Willis and P. Rehak.

[66] E. Aprile, K. L. Giboni and C. Rubbia, Nucl. Instrum. Meth. A253, 273 (1987).

[67] C.Arroyo, B.J.King et. al., Phys. Rev. Lett. 72, 3452 (1994).

[68] K.S.McFarland et al., CCFR, Eur. Phys. Jour. C1, 509 (1998)

[69] C. H. Llewellyn Smith, Nucl. Phys. B228, 205 (1983).

[70] E. A. Paschos and L. Wolfenstein, Phys. Rev. D 7, 91 (1973).

[71] K.S.McFarland et. al., NuTeV collaboration, Proceedings of the XXXIIIrd Rencontres de Moriond (1998), hep-ex/9806013;

J. Yu et. al., NuTeV collaboration, proceedings of the DIS98, Brussels, Belgium, Eds. G.H.Coremans and R. Roosen, World Scientific, 588 (1998).

[72] U.K Yang and A. Bodek, UR-1543, Submitted to Phys. Rev. Lett, hepex/9809480 (1998)

[73] E. Sather, Phys. Lett. B274 (1992) 433.

[74] D. Yu. Bardin and V. A. Dokuchaeva, JINR E2-86-260 (1986).

[75] U. Baur, "Electroweak Radiative Corrections to $W$ Boson Production at the Tevatron", SUNY-Buffalo preprint UB-HET-98-02, Sep 1998 (e-Print Archive: hep-ph/9809327).

[76] U. Baur and M. Demarteau, "Precision Electroweak Physics at Future Collider Experiments," Fermilab-Conf-96/423 (1996).

[77] J. F. Donoghue and E. Golowich, Phys. Rev. D49, 1513 (1994) hep$\mathrm{ph} / 9307262$.

[78] J. F. Donoghue and E. Golowich, Phys. Lett. B478, 172 (2000) hepph/9911309.

[79] D. Geiregat et al. [CHARM-II Collaboration], Phys. Lett. B 245, 271 (1990). 
[80] S. R. Mishra et al. [CCFR Collaboration], Phys. Rev. Lett. 66, 3117 (1991).

[81] W. Czyz, G.C. Sheppey, and J.D. Walecka, Nuovo Cim. 34, 404 (1964).

[82] R. Belusevic and J. Smith, Phys. Rev. D37, 2419 (1988).

[83] Y. Grossman, Z. Ligeti and E. Nardi, Nucl. Phys. B465, 369 (1996) hep$\mathrm{ph} / 9510378$.

[84] A. J. Buras and R. Fleischer, hep-ph/9704376.

[85] D. Zeppenfeld and K. Cheung, hep-ph/9810277.

[86] M. Gronau, C.N. Leung and J.L. Rosner, Phys. Rev. D29, 2539 (1984).

[87] R. N. Mohapatra and G. Senjanovic, Phys. Rev. D23, 165 (1981).

[88] D. Wyler and L. Wolfenstein, Nucl. Phys. B218, 205 (1983).

[89] L. M. Johnson, D. W. McKay and T. Bolton, Phys. Rev. D56, 2970 (1997) hep-ph/9703333.

[90] A. Vaitaitis et al. [NuTeV Collaboration], Phys. Rev. Lett. 83, 4943 (1999) .

[91] J. A. Formaggio et al. [NuTeV Collaboration], Phys. Rev. Lett. 84, 4043 (2000)

[92] T. Adams et al. [NuTeV Collaboration], hep-ex/0104037. .

[93] N. Ushida et al. (E531 Collaboration), Phys. Lett. B206 (1988), 375.

[94] R. E. Shrock and B. W. Lee, Phys. Rev. D 13, 2539 (1976).

[95] Tim Bolton, E803 Physics, unpublished notes, KSU HEP 95-02.

[96] X. H. Guo and A. W. Thomas, Phys. Rev. D 61, 116009 (2000) hep$\mathrm{ph} / 9907370$.

[97] E. M. Aitala et al. [E791 Collaboration], charmed mesons," Phys. Lett. B462, 401 (1999) hep-ex/9906045.

A second E791 rare charm decay paper has recently been accepted by PRL and is available at Los Alamos as hep-ex 0011077. *

[98] E. M. Aitala et al. [E791 Collaboration], D0 -i. V l+ l- and h h l l," hepex/0011077.

[99] M. A. Shifman and M. B. Voloshin, Sov. J. Nucl. Phys. 41, 120 (1985).

[100] J. Chay, H. Georgi and B. Grinstein, Phys. Lett. B247, 399 (1990).

[101] I. I. Bigi, N. G. Uraltsev and A. I. Vainshtein, Phys. Lett. B293, 430 (1992) hep-ph/9207214.

[102] B. Blok, L. Koyrakh, M. Shifman and A. I. Vainshtein, Phys. Rev. D49, 3356 (1994) hep-ph/9307247. 
[103] A. F. Falk, M. Luke and M. J. Savage, Phys. Rev. D53, 6316 (1996) hepph/9511454.

[104] G. Burdman, E. Golowich, J. L. Hewett and S. Pakvasa, Phys. Rev. D52, 6383 (1995) hep-ph/9502329.

[105] C. Greub, T. Hurth, M. Misiak and D. Wyler, Phys. Lett. B382, 415 (1996) hep-ph/9603417.

[106] J. Anjos, et al., Phys. Rev. Lett. 60, (1988) 1239.

[107] E791 Collaboration, Phys. Rev. Lett. 77, (1996) 2384; Phys. Rev. D57 (1998) 13.

[108] R. Godang et al. [CLEO Collaboration], Phys. Rev. Lett. 84, 5038 (2000) hep-ex/0001060.

[109] H. Park, hep-ex/0005044.

[110] A. F. Falk, Y. Nir and A. A. Petrov, "Strong phases and D0 anti-D0 mixing parameters," JHEP 9912, 019 (1999) hep-ph/9911369.

[111] J. M. Link et al. [FOCUS Collaboration], Phys. Lett. B485, 62 (2000) hep$\mathrm{ex} / 0004034$.

[112] S. Bergmann, Y. Grossman, Z. Ligeti, Y. Nir and A. A. Petrov, Phys. Lett. B486, 418 (2000) hep-ph/0005181.

[113] For a comprehensive review of $B$ physics at the $\Upsilon(4 S)$, see P. F. Harrison and H. R. Quinn [BABAR Collaboration], The BaBar physics book: Physics at an asymmetric B factory, SLAC-R-0504, 1998.

[114] . I. Bigi and N. G. Uraltsev, Nucl. Phys. B592, 92 (2000) hep-ph/0005089.

[115] E. Golowich and A. A. Petrov, Phys. Lett. B427, 172 (1998) hep-ph/9802291. For a review see: G. Burdman, in: "Workshop on the Tau/Charm Factory", Argonne National Lab, 1995, AIP Conference Proceedings No. 349, p.409, or A. A. Petrov, hep-ph/0009160.

[116] J. Ellis and D. V. Nanopoulos, Phys. Lett. B110, 44 (1982). F. Gabbiani, E. Gabrielli, A. Masiero and L. Silvestrini, Nucl. Phys. B477, 321 (1996) hep$\mathrm{ph} / 9604387$.

[117] Y. Nir and N. Seiberg, Phys. Lett. B309 (1993) 337; M. Leurer, Y. Nir and N. Seiberg, Nucl. Phys. B420 (1994) 468.

[118] G.C. Branco, et al., Phys. Rev. D52 (1995) 4217.

[119] S. Davisdon, et al., Z. Phys. C61 (1994) 613.

[120] L.F. Abbot, et al. Phys. Rev. D21 (1980) 1393; Y. Grossman, Nucl. Phys. B426 (1994) 355. 
[121] S. Pakvasa and H. Sugawara, Phys. Lett. B73 (1978) 61; T.P. Chen and M. Sher, Phys. Rev. D35 (1987) 3484; L. Hall and S. Weinberg, Phys. Rev. D48 (1993) R979; D. Atwood, et al. Phys. Rev. D55 (1997) 3156.

[122] I.I. Bigi, in: Proc. XIII Int. Conf. on High Energy Physics, S.C. Loken (ed.), World Scientific, Singapore, 1986, p. 857; G. Blaylock, A. Seiden and Y. Nir, Phys. Lett. B 355 (1995) 555.

[123] F. Buccella, M. Lusignoli and A. Pugliese, Phys. Lett. B379, 249 (1996) hep$\mathrm{ph} / 9601343$.

[124] D. Benson, I.I. Bigi and A.I. Sanda, in preparation.

[125] Fits and references to the Homestake, Kamiokande, GALLEX, SAGE, and Super Kamiokande data include N. Hata and P. Langacker, Phys. Rev. D56 6107 (1997); J. Bahcall, P. Krastev, and A. Smirnov, Phys. Rev. D58, 096016 (1998); J. Bahcall and P. Krastev, Phys. Lett. B436, 243 (1998); J. Bahcall, P. Krastev, and A. Smirnov, Phys. Rev. D60, 093001 (1999), http://www.sns.ias.edu/ jnb/, and M. Gonzalez-Garcia, P. de Holanda, C. Pena-Garay, and J. W. F. Valle, Nucl. Phys. B, in press (hep-ph/9906469). Recent Super Kamiokande data is reported in Super Kamiokande Collab., Y.Fukuda et al., Phys. Rev. Lett. 82, 1810, 243 (1999).

[126] L. Wolfenstein, Phys. Rev. D17, 2369 (1978).

[127] S. P. Mikheyev and A. Smirnov, Yad. Fiz. 42, 1441 (1985) [Sov.J. Nucl. Phys. 42, 913 (1986)], Nuovo Cim., C9, 17 (1986).

[128] Kamiokande Collab., K. S. Hirata, Phys. Lett. B205, 416; ibid. 280, 146 (1992); Y.Fukuda et al., Phys. Lett. B335, 237 (1994); S. Hatakeyama et al. Phys. Rev. Lett. 81, 2016 (1998).

[129] IMB Collab., D. Casper et al., Phys. Rev. Lett. 66, 2561 (1991); R.BeckerSzendy et al., Phys. Rev. D46, 3720 (1992); Phys. Rev. Lett. 69, 1010 (1992).

[130] Super-Kamiokande Collab., Y. Fukuda et al., Phys. Lett. B433, 9 (1998); Phys. Rev. Lett. 81,1562 (1998); ibid., 82, 2644 (1999); Phys. Lett. B467, 185 (1999).

[131] Soudan Collab., W. Allison et al, Phys. Lett. B391, 491 (1997); Soudan-2 Collab., Phys. Lett. B449, 137 (1999); A. Mann, in Proceedings of the 1999 Photon-Lepton Symposium, hep-ex/9912007.

[132] MACRO Collab., M. Ambrosio et al., Phys. Lett. B434, 451 (1998); hepex/0001044.

[133] J. Learned, in the Proceedings of the Workshop on the Next Generation Nucleon Decay and Neutrino Detector NNN99, Stony Brook (Sept. 1999).

[134] M. Apollonio et al., Phys. Lett. B420, 397 (1998); Phys. Lett. B466, 415 (1999). 
[135] For recent experimental reviews, see, e.g., L. DiLella, hep-ex/9912010; H. Robertson, hep-ex/0001034, and talks at the Workshop on the Next Generation Nucleon Decay and Neutrino Detector NNN99, Stony Brook (Sept. 1999), http://superk.physics.sunysb.edu/NNN99/scientific_program/.

[136] LSND Collab., C. Athanassopoulous et al., Phys. Rev. Lett. 77, 3082 (1996), LSND Collab., C. Athanassopoulous et al., Phys. Rev. Lett. 81, 1774 (1998).

[137] KARMEN Collab., K. Eitel, B. Zeitnitz, in Proceedings of Neutrino-98, Nucl. Phys. (Proc. Suppl.) 77, 212 (1999).

[138] References and websites for these experiments and future projects can be found, e.g., at http://www.hep.anl.gov/ndk/hypertext/nu_industry.htm.

[139] ICANOE Collab. F. Cavanna et al., LNGS-P21-99-ADD-1,2, Nov 1999; A. Rubbia, hep-ex/0001052.

[140] OPERA Collab., CERN-SPSC-97-24, hep-ex/9812015.

[141] V. Barger, K. Whisnant, S. Pakvasa, and R. J. N. Phillips, Phys. Rev. D22, 2718 (1980). See also V. Barger, K. Whisnant, and R. J. N. Phillips, Rev. Rev. Lett. 45, 2084 (1980).

[142] D. Ayres, T. Gaisser, A. K. Mann, and R. Shrock, in Proceedings of the 1982 DPF Summer Study on Elementary Particles and Future Facilities, Snowmass, p. 590; D. Ayres, B. Cortez, T. Gaisser, A. K. Mann, R. Shrock, and L. Sulak, Phys. Rev. D29, 902 (1984).

[143] P. Krastev, S. Petcov, Phys. Lett. B205, 8 (1988).

[144] A. J. Baltz, J. Weneser, Phys. Rev. D37, 3364 (1988).

[145] C. W. Kim and A. Pevsner, Neutrinos in Physics and Astrophysics (Harwood, Langhorne, 1993).

[146] S. Petcov, Phys. Lett. B434, 321 (1998). M. Chizhov, M. Maris, S. Petcov, hep-ph/9810501; M. Chizhov, S. Petcov, hep-ph/9903424; M.Chizhov, S.Petcov, Phys. Rev. Lett. 83, 1096 (1999).

[147] E. Akhmedov, A. Dighe, P. Lipari, A. Smirnov, Nucl. Phys. B542, 3 (1999); E. Akhmedov, Nucl.Phys. B538, 25 (1999); hep-ph/0001264.

[148] P. Krastev, Nuovo Cimento 103A, 361 (1990). R. H. Bernstein and S. J. Parke, Phys. Rev. D44, 2069 (1991).

[149] De Rujula, M. B. Gavela, and P. Hernandez, Nucl. Phys. B547, 21 (1999).

[150] M. Campanelli, A. Bueno and A. Rubbia, hep-ph/9905240.

[151] D. Dooling, C. Giunti, K. Kang and C. W. Kim, Phys. Rev. D 61, 073011 (2000) hep-ph/9908513.

[152] I. Mocioiu, R. Shrock, in the Proceedings of the Workshop on the Next Generation Nucleon Decay and Neutrino Detector NNN99, Stony Brook (Sept. 1999), hep-ph/9910554. 
[153] I. Mocioiu and R. Shrock, Phys. Rev. D 62, 053017 (2000) hep-ph/0002149.

[154] S.M. Bilenky, C. Giunti, W.Grimus, Phys.Rev.D58, 033001 (1998); K. Dick, M. Freund, M. Lindner, A. Romanino, Nucl. Phys. B562, 29 (1999); M. Tanimoto, Phys. Lett. B462, 115 (1999); A. Donini, M. B. Gavela, P. Hernandez and S. Rigolin, Nucl. Phys. B574, 23 (2000) [hep-ph/9909254; M. Koike and J. Sato, Phys. Rev. D 61, 073012 (2000) hep-ph/9909469 ; P. F. Harrison and W. G. Scott, Phys. Lett. B476, 349 (2000) hep-ph/9912435;

[155] M. Freund, M. Lindner, S. T. Petcov and A. Romanino, Nucl. Phys. B578, 27 (2000) hep-ph/9912457.

[156] The MINOS experiment, http://www-numi.fnal.gov:8875.

[157] The ICANOE experiment, http://pcnometh4.cern.ch.

[158] A. Villanueva et al, Nufact'99 Workshop,July 5-9th, Lyon 1999.

[159] Doke, et al., Nucl. Instrum. Meth A237 475 (1985)

[160] V. Barger, S. Geer, K. Whisnant, Phys.Rev. D61, 053004 (2000).

[161] A. Cervera, A. Donini, M. B. Gavela, J. J. Gomez Cadenas, P. Hernandez, O. Mena and S. Rigolin, Nucl. Phys. B579, 17 (2000) hep-ph/0002108.

[162] P. Strolin, Nufact'99 Workshop,July 5-9th, Lyon 1999

[163] D. A. Harris and A. Para, Nucl. Instrum. Meth. A451, 173 (2000) hep$\mathrm{ex} / 0001035$. 